Supporting Information for:

\title{
Metal-Free, Visible Light-Photocatalyzed Synthesis of Benzo[b]phosphole Oxides: Synthetic and Mechanistic Investigations
}

Valentin Quint, ${ }^{\dagger}$ Fabrice Morlet-Savary, ${ }^{\ddagger}$ Jean-François Lohier,${ }^{\dagger}$ Jacques Lalevée, ${ }^{\ddagger}$ Annie-Claude Gaumont, ${ }^{\dagger}$ and Sami Lakhdar ${ }^{\star \dagger}$

${ }^{\dagger}$ Normandie University, LCMT, ENSICAEN, UNICAEN, CNRS, 14000-France

¥Institut de Science des Matériaux de Mulhouse IS2M - UMR CNRS 7361 - UHA, 15, rue Jean Starcky, 68057 Mulhouse Cedex, France.

*Correspondence to: sami.lakhdar@ensicaen.fr 


\section{Table of contents}

I. General information $\quad$ S3

II. Synthesis of starting materials $\quad$ S5

III. General procedure for the organophotocatalyzed formation S6 of benzophosphole derivatives

III.1. Optimization of reaction conditions $\quad$ S6

III.2. Control experiment $\quad$ S7

III.3. Synthesis of the benzophosphole derivatives S8

IV. Mechanistic investigations $\quad$ S25

IV.1. Determination of the quantum yield of the photoreaction $\quad$ S25

$\begin{array}{ll}\text { IV.2. EPR Spin Trapping } & \text { S25 }\end{array}$

$\begin{array}{ll}\text { IV.3. Fluorescence quenching } & \text { S27 }\end{array}$

$\begin{array}{ll}\text { IV.4. Photolysis study } & \text { S28 }\end{array}$

V. X-ray data $\quad$ S29

$\begin{array}{ll}\text { VI. References } & \text { S30 }\end{array}$

VII. NMR data $\quad$ S32 


\section{General information}

All solvents were reagent grade. Toluene, THF, diethyl ether, acetonitrile and dichloromethane were purified by an Innovative Technology PURESOLV ${ }^{\circledR}$ purification

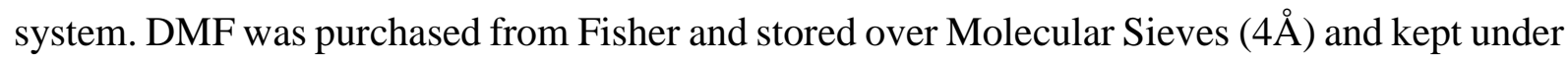
argon. Flash column chromatography were carried out with Merck silica gel 60 (0.040-0.063 $\mathrm{mm})$. Chromatography fractions and stated reactions were monitored by TLC on Merck silica gel 60 F254 aluminum plates. The spots were visualized under UV light at $254 \mathrm{~nm}$ and $366 \mathrm{~nm}$ and treated with aqueous $\mathrm{KMnO}_{4}$ solution followed by heating with a heat gun.

${ }^{1} \mathrm{H},{ }^{11} \mathrm{~B},{ }^{13} \mathrm{C},{ }^{19} \mathrm{~F}$ and ${ }^{31} \mathrm{P}$ NMR spectra were recorded on a Bruker $400 \mathrm{MHz}$ or $500 \mathrm{MHz}$ spectrometers in $\mathrm{CDCl}_{3}, \mathrm{CD}_{3} \mathrm{OD}$ or $\mathrm{d}_{6}$-DMSO. Chemical shifts are reported in ppm relative to the residual signals of the deuterated solvents as the internal standard $\left(\mathrm{CDCl}_{3}: \delta_{\mathrm{H}}=7.26, \delta_{\mathrm{C}}=\right.$ 77.16; $\left.\mathrm{CD}_{3} \mathrm{OD}, \delta_{\mathrm{H}}=3.31 ;\left(\mathrm{CD}_{3}\right)_{2} \mathrm{SO}: \delta_{\mathrm{H}}=2.50, \delta_{\mathrm{C}}=39.52 \mathrm{ppm}\right) .{ }^{13} \mathrm{C}$ NMR spectra were recorded using an internal reference, ${ }^{31} \mathrm{P}$ NMR spectra were recorded using $85 \% \mathrm{H}_{3} \mathrm{PO}_{4}$ as external reference, ${ }^{11} \mathrm{~B}$ NMR spectra were recorded using $\mathrm{BF}_{3} \cdot \mathrm{Et}_{2} \mathrm{O}$ as external reference and ${ }^{19} \mathrm{~F}$ NMR spectra were recorded using $\mathrm{CFCl}_{3}$ as external reference. Multiplicities are indicated by s (singlet), d (doublet), t (triplet), q (quartet), m (multiplet), and br (broad). Coupling constants, $J$, are reported in Hertz. Assignments were obtained by reference to 2D-NMR experiments (COSY, HSQC, NOESY, and HMBC). High-resolution mass-spectra were obtained on a Waters Qtof Micro spectrometer. UV-Visible spectra were recorded on a Perkin Elmer Lambda 40 Spectrophotometer.

All reactions were carried out under a protective atmosphere of dry nitrogen using oven-dried glassware unless otherwise stated.

The green light irradiation was performed using high-power LEDs Vision-EL $(5 \mathrm{~W}, \lambda=530 \pm 15$ $\mathrm{nm}, 410 \mathrm{~lm})$. 


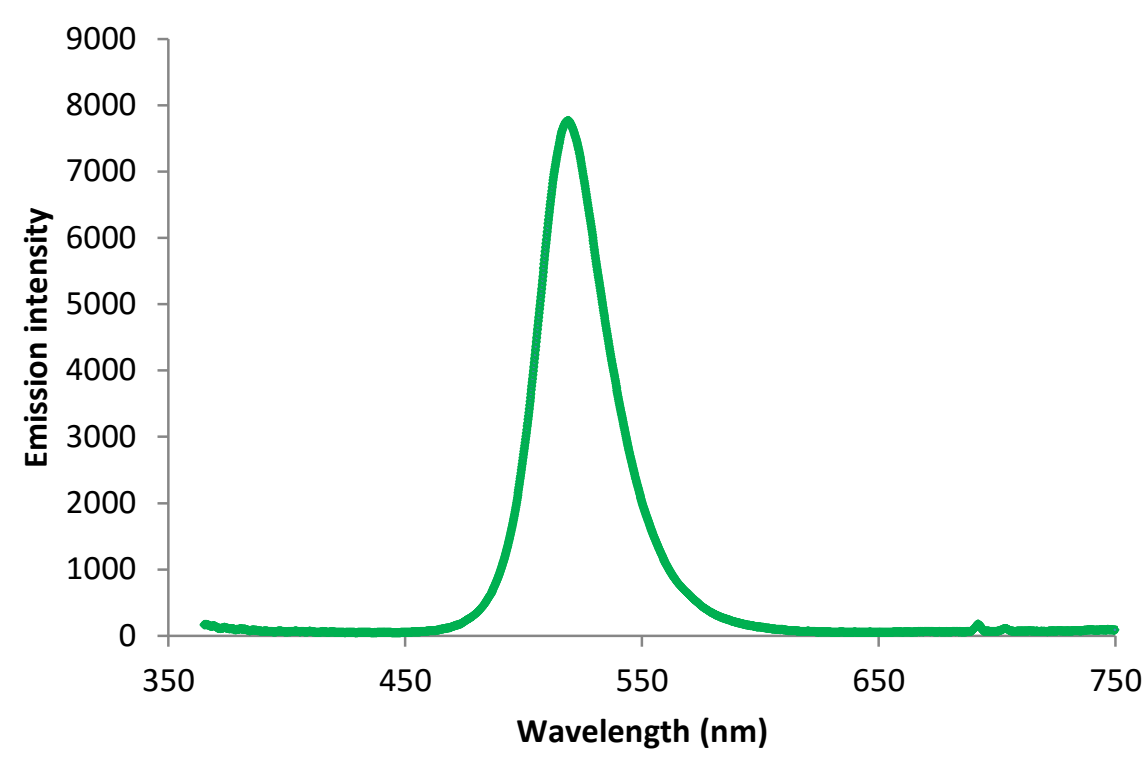

Figure S1. Emission spectrum of the green LED lamp used in our experiments.

The blue light irradiation was performed using high-power Vision-EL $(5 \mathrm{~W}, \lambda=460 \pm 10 \mathrm{~nm}$, $410 \mathrm{~lm})$.

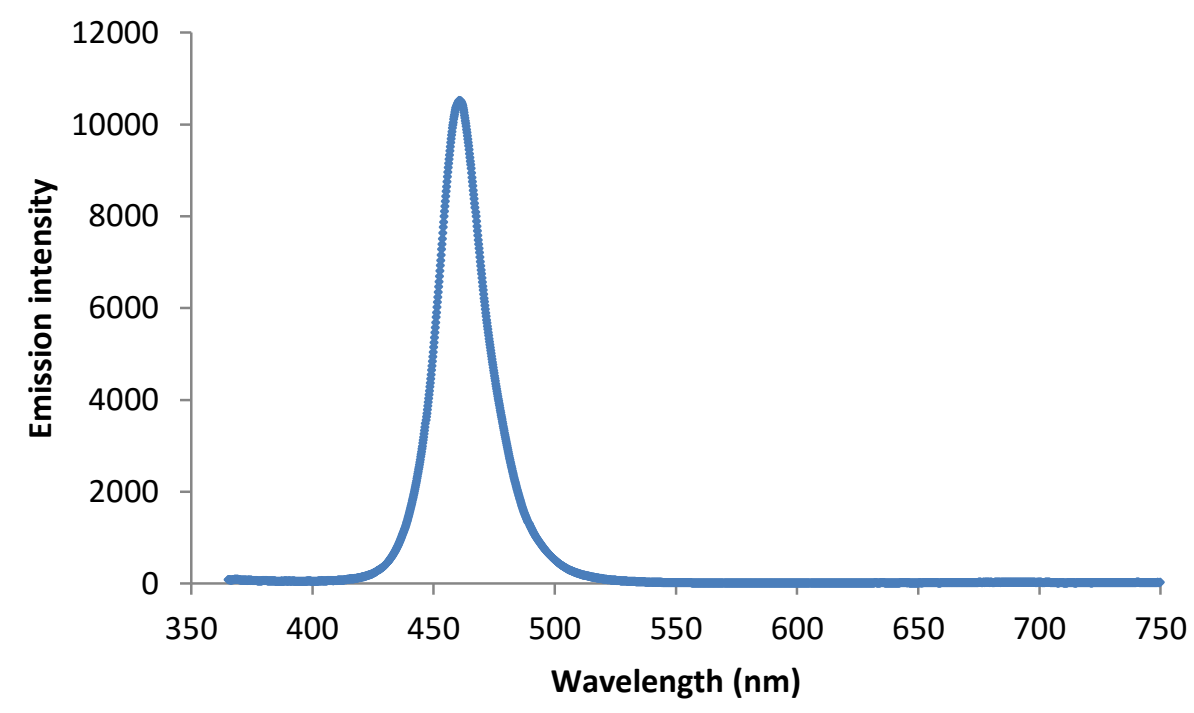

Figure S2. Emission spectrum of the blue LED lamp used in the screening experiments.

EPR-ST experiments were carried out using an X-Band spectrometer (MS 400 Magnettech). The EPR spectra simulations were carried out using the WINSIM software.

Fluorescence measurements were performed on a JASCO FP-6200 Spectro Fluorimeter.

Photolysis experiments were done on a JASCO V-730 UV/vis spectrophotometer. 


\section{Synthesis of starting materials}

Diphenyl iodonium triflate $\mathbf{8 c},{ }^{[1]}$ symmetrical alkynes $\mathbf{2 b - 2 e},^{[2]}$ dissymmetrical alkynes $\mathbf{2 j}$, ${ }^{[3]}$ $\mathbf{2 l},{ }^{[4]}$ and secondary phosphine oxides $\mathbf{1 m - 1 n},{ }^{[5]} \mathbf{1 0 - 1 x},{ }^{[6]} \mathbf{1} \mathbf{r}^{[7]}$ were synthesized following reported procedures.

Triphenylsulfonium tetrafluoroborate (8a) was synthesized by a procedure reported by Akiyami. ${ }^{[8]}$

$\mathrm{N}$-ethoxy-2-methylpyridinium tetrafluoroborate (8b) was synthesized according to a protocol by Reichardt. ${ }^{[9]}$

All other chemicals were purchased from Sigma Aldrich, Alfa Aesar or Fisher.

\section{Preparation of diphenylphosphine oxide (1a):}

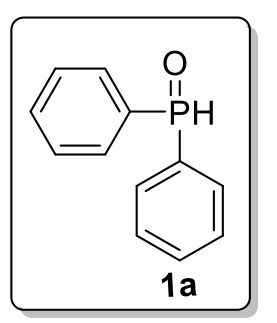

In a two necked round bottom flask flushed with argon, chlorodiphenylphosphine ( $45 \mathrm{mmol}, 10 \mathrm{~g}$ ) is dissolved in dry acetonitrile $(40 \mathrm{~mL})$ at $0^{\circ} \mathrm{C}$. Under stirring, $20 \mathrm{~mL}$ of degassed distilled water is added slowly and the reaction mixture is allowed to warm at $40^{\circ} \mathrm{C}$ for $2 \mathrm{~h}$. After cooling down to room temperature, dichloromethane $(30 \mathrm{~mL})$ is added. The aqueous phase is then extracted twice with dichloromethane $(2 \times 30 \mathrm{~mL})$. The combined organic phases were washed with saturated $\mathrm{NaHCO}_{3}(50 \mathrm{~mL})$, dried over $\mathrm{MgSO}_{4}$ and then concentrated under reduced pressure. The residue is filtered on a pad of silica with $50 \mathrm{~mL}$ of DCM and the collected fraction are then concentrated under reduced pressure to afford the desired product as a white solid (7.7 g, $86 \%)$.

${ }^{1} \mathrm{H} \mathrm{NMR}\left(400 \mathrm{MHz}, \mathrm{CDCl}_{3}\right): \delta=7.47-7.58(\mathrm{~m}, 6 \mathrm{H}), 7.67-7.74(\mathrm{~m}, 4 \mathrm{H}), 7.98(\mathrm{~d}, J=481.0 \mathrm{~Hz})$. ${ }^{13} \mathrm{C}\left\{{ }^{1} \mathrm{H}\right\} \mathrm{NMR}\left(100 \mathrm{MHz}, \mathrm{CDCl}_{3}\right): \delta=129.0(\mathrm{~d}, J=12.7 \mathrm{~Hz}), 130.9(\mathrm{~d}, 11.4 \mathrm{~Hz}), 131.6(\mathrm{~d}, J$ $=101.0 \mathrm{~Hz}), 132.7(\mathrm{~d}, J=2.4 \mathrm{~Hz})$.

${ }^{31} \mathrm{P}\left\{{ }^{1} \mathrm{H}\right\} \mathrm{NMR}\left(162 \mathrm{MHz}, \mathrm{CDCl}_{3}\right): \delta=21.5(\mathrm{~d}, J=481.0 \mathrm{~Hz})$.

HRMS (ESI) m/z calcd for $\mathrm{C}_{12} \mathrm{H}_{12} \mathrm{OP}(\mathrm{M}+\mathrm{H})^{+}: 203.0620$, found : 203.0615. 


\section{General procedure for the organophotocatalyzed formation of}

\section{benzophospholes derivatives}

\section{III.1. Optimization of reaction conditions}

Table S1 : Identification of optimal reaction conditions. ${ }^{\text {[a] }}$

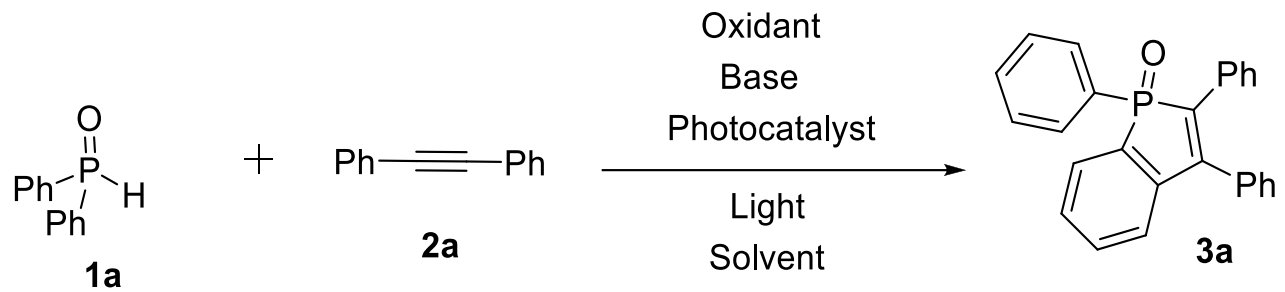

\begin{tabular}{|c|c|c|c|c|c|c|}
\hline Entry & Oxidant & Photocatalyst & Base & Solvent & Light & $\begin{array}{c}\text { NMR Yield } \\
(\%)^{[\mathrm{b}]}\end{array}$ \\
\hline 1 & $8 \mathbf{a}$ & EOSIN Y 9c & $\mathrm{NaHCO}_{3}$ & DMF & Green LEDs & 62 \\
\hline 2 & $8 b$ & EOSIN Y 9c & $\mathrm{NaHCO}_{3}$ & DMF & Green LEDs & 75 \\
\hline 3 & $8 c$ & EOSIN Y 9c & $\mathrm{NaHCO}_{3}$ & DMF & Green LEDs & 45 \\
\hline 4 & $8 b$ & Fluorescein 9b & $\mathrm{NaHCO}_{3}$ & DMF & Green LEDs & 37 \\
\hline 5 & $8 b$ & Rhodamine B 9a & $\mathrm{NaHCO}_{3}$ & DMF & Green LEDs & 25 \\
\hline 6 & $8 b$ & EOSIN Y 9c & $\mathrm{NaOAc}$ & DMF & Green LEDs & 52 \\
\hline 7 & $\mathrm{BrCCl}_{3}$ & EOSIN Y 9c & $\mathrm{NaHCO}_{3}$ & DMF & Green LEDs & 0 \\
\hline 8 & $8 b$ & EOSIN Y 9c & $\mathrm{NaHCO}_{3}$ & Acetonitrile & Green LEDs & 39 \\
\hline 9 & $8 b$ & Rhodamine B 9a & $\mathrm{NaHCO}_{3}$ & DMSO & Green LEDs & 70 \\
\hline 10 & $8 b$ & No & $\mathrm{NaHCO}_{3}$ & DMF & Green LEDs & 4 \\
\hline 11 & $8 b$ & No & $\mathrm{NaHCO}_{3}$ & DMF & Blue LEDs & 8 \\
\hline 12 & $8 b$ & EOSIN Y 9c & No & DMF & Green LEDs & 24 \\
\hline 13 & No & EOSIN Y 9c & $\mathrm{NaHCO}_{3}$ & DMF & Green LEDs & 0 \\
\hline 14 & $8 b$ & EOSIN Y 9c & $\mathrm{NaHCO}_{3}$ & DMF & No light ${ }^{[\mathrm{c}]}$ & 0 \\
\hline 15 & $8 b$ & EOSIN Y 9c & $\mathrm{NaHCO}_{3}$ & DMF & Green LEDs & $95^{[\mathrm{d}]}$ \\
\hline 16 & $8 b$ & EOSIN Y 9c & $\mathrm{NaHCO}_{3}$ & DMF & Green LEDs & $79^{[\mathrm{e}]}$ \\
\hline
\end{tabular}

[a] Reaction conditions : secondary phosphine oxide 1a $(0.5 \mathrm{mmol})$, alkyne $\mathbf{2 a}(0.5 \mathrm{mmol})$, oxidant $(0.75 \mathrm{mmol})$, base $(0.6 \mathrm{mmol})$, photocatalyst $(0.02 \mathrm{mmol})$, solvent $(5 \mathrm{~mL}), 48 \mathrm{~h}$. [b] NMR yields are determined from ${ }^{31} \mathrm{P}$ NMR spectroscopy using trioctylphosphine oxide as internal standard. [c] The reaction is protected from external light with foil and heated at $35{ }^{\circ} \mathrm{C}$ to reproduce the heat caused by the LED lamp. [d] Reaction conditions: secondary phosphine oxide 1a $(1 \mathrm{mmol})$, Alkyne $2 \mathbf{a}(0.5 \mathrm{mmol})$, oxidant $(1 \mathrm{mmol})$, base $(0.6 \mathrm{mmol})$, photocatalyst $(0.02$ 
mmol), solvent (5 mL), 48h. [e] Reaction conditions: Secondary phosphine oxide 1a (0.5 mmol), Alkyne 2a (1 mmol), oxidant $(0.75 \mathrm{mmol})$, base $(0.6 \mathrm{mmol})$, photocatalyst $(0.02 \mathrm{mmol})$, solvent $(5 \mathrm{~mL}), 48 \mathrm{~h}$.

\section{III.2. Control experiment}

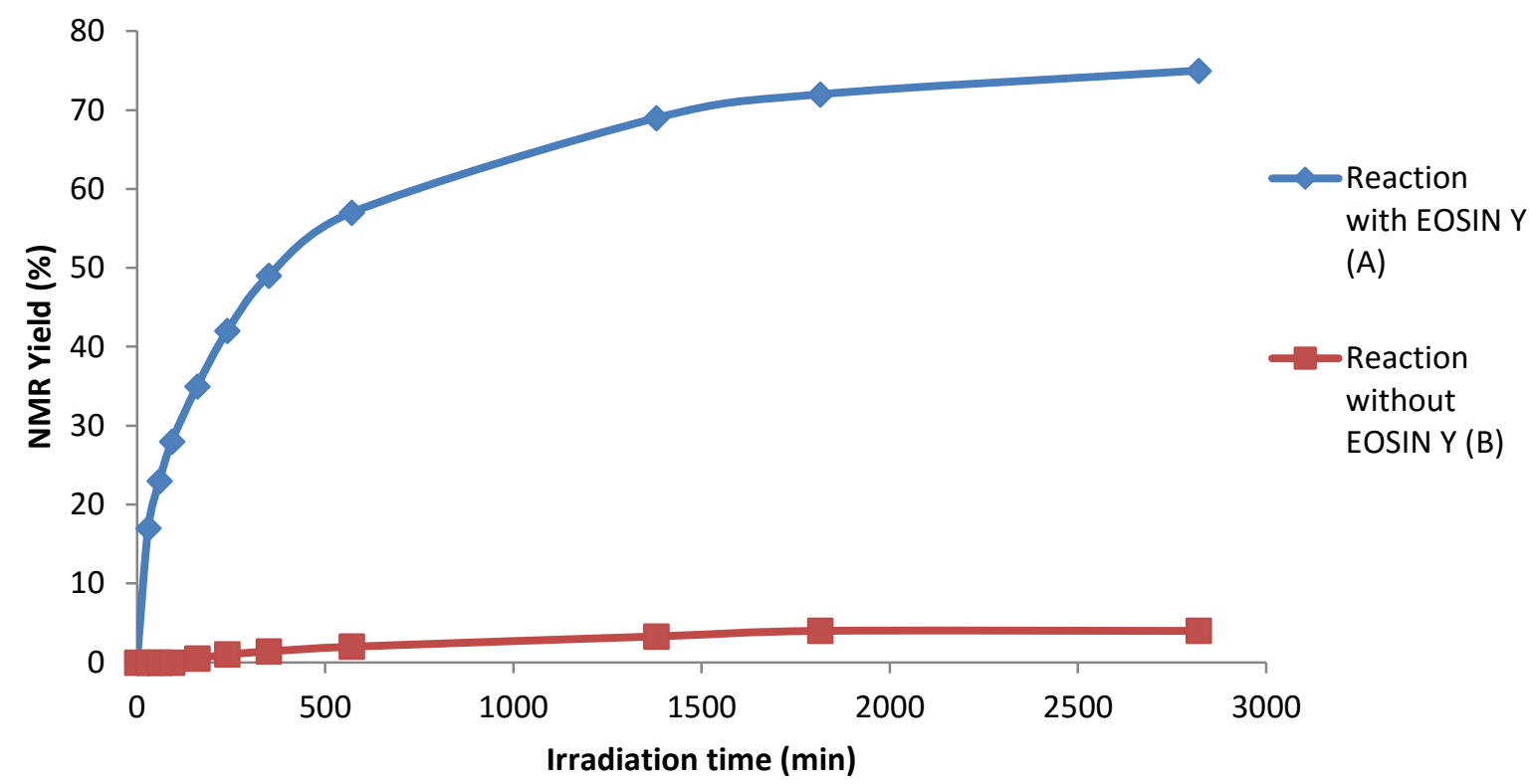

Figure S3. Reaction progress in the presence of eosin Y (curve A), and in the absence of eosin $\mathrm{Y}$ (curve B). ${ }^{\text {a] }}$

[a] Reaction conditions : Secondary phosphine oxide 1a $(0.5 \mathrm{mmol})$, alkyne $\mathbf{2 a}(0.5 \mathrm{mmol}), \mathbf{8 b}(0.75 \mathrm{mmol})$, $\mathrm{NaHCO}_{3}(0.6 \mathrm{mmol})$, photocatalyst $(0.02 \mathrm{mmol})$ in DMF $(5 \mathrm{~mL})$ (curve A) or no photocatalyst (curve B). NMR yields are determined from ${ }^{31} \mathrm{P}$ NMR spectroscopy using trioctylphosphine oxide as internal standard. 


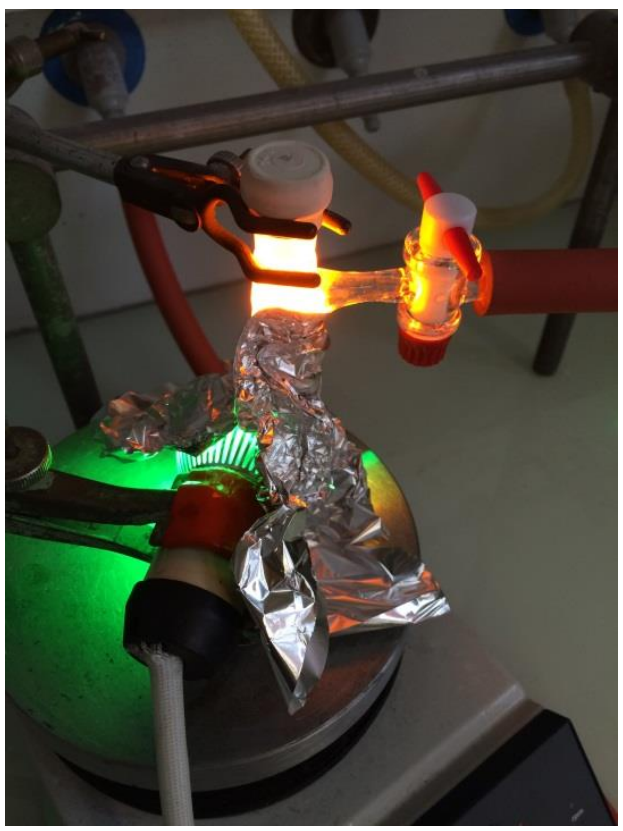

Figure S4. Set-up for a typical organophotocatalyzed reaction.

\section{III.3. Synthesis of benzophosphole derivatives}

\section{General procedure for the organophotocatalysed formation of phospholes (GP):}

A dry and argon-flushed Schlenk-flask, equipped with a magnetic stirring bar and a septum is charged with sodium hydrogenocarbonate (50 mg, $0.60 \mathrm{mmol}, 1.2$ eq.), $N$-ethoxy-2methylpyridinium tetrafluoroborate $(225 \mathrm{mg}, 1.0 \mathrm{mmol}, 2 \mathrm{eq}$.$) , secondary phosphine oxide (1.0$ mmol, 2 eq.), alkyne (0.50 mmol, 1 eq.) and eosin Y (13 mg, $0.020 \mathrm{mmol}, 0.04$ eq.). Dry DMF $(8.0 \mathrm{~mL})$ is then added and the reaction mixture is irradiated with a green LED lamp $(5 \mathrm{~W})$. After stirring for the indicated time, the crude mixture was purified by flash column chromatography to afford the title compound in the stated yield.

\section{1,2,3-Triphenyl-1H-phosphindole-1-oxide (3a):}

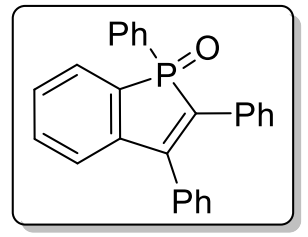

According to GP, sodium hydrogenocarbonate $(50 \mathrm{mg}, 0.60 \mathrm{mmol}, 1.2$ eq.), $N$-ethoxy-2-methylpyridinium tetrafluoroborate $(225 \mathrm{mg}, 1.0 \mathrm{mmol}$, 2 eq.), diphenylphosphine oxide (202 mg, $1.0 \mathrm{mmol}, 2$ eq.), diphenylacetylene (89 mg, $0.50 \mathrm{mmol}, 1$ eq.) and eosin $\mathrm{Y}(13 \mathrm{mg}, 0.02$ mmol, 0.04 eq.) were dissolved in DMF and irradiated for $48 \mathrm{~h}$. Purification by flash chromatography (silica gel, $n$-pentane/EtOAc : 1/1) afforded the benzophosphole oxide 3a (147 mg, 78\%) as a white solid. 
${ }^{1} \mathrm{H}$ NMR (400 MHz, $\left.\mathrm{CDCl}_{3}\right): \delta=7.13-7.06(\mathrm{~m}, 3 \mathrm{H}), 7.25-7.19(\mathrm{~m}, 3 \mathrm{H}), 7.52-7.30(\mathrm{~m}, 10 \mathrm{H})$, $7.71(\mathrm{dd}, J=8.8 \mathrm{~Hz} ; J=7.6 \mathrm{~Hz}, 1 \mathrm{H}), 7.78(\mathrm{dd}, J=12.4 \mathrm{~Hz} ; J=7.6 \mathrm{~Hz}, 2 \mathrm{H})$.

${ }^{13} \mathrm{C}\left\{{ }^{1} \mathrm{H}\right\} \mathrm{NMR}\left(100 \mathrm{MHz}, \mathrm{CDCl}_{3}\right): \delta=124.1(\mathrm{~d}, J=10.6 \mathrm{~Hz}), 127.9,128.4,128.8(\mathrm{~d}, J=12.1$ Hz), 128.9, 129.02, 129.08, 129.1, 129.2, 129.3, 130.0 (d, $J=98.7 \mathrm{~Hz}), 131.1$ (d, $J=10.6 \mathrm{~Hz})$, 131.6, 132.2 (d, $J=108.4 \mathrm{~Hz}), 132.3(\mathrm{~d}, J=3.1 \mathrm{~Hz}), 132.7$ (d, $J=9.8 \mathrm{~Hz}), 133.0$ (d, $J=1.9$ $\mathrm{Hz}), 134.32(\mathrm{~d}, J=96.2 \mathrm{~Hz}), 134.37(\mathrm{~d}, J=15.2 \mathrm{~Hz}), 143.8(\mathrm{~d}, J=26.8 \mathrm{~Hz}), 150.1$ (d, $J=21.1$ $\mathrm{Hz})$.

${ }^{31} \mathrm{P}\left\{{ }^{1} \mathrm{H}\right\}$ NMR $\left(162 \mathrm{MHz}, \mathrm{CDCl}_{3}\right): \delta=39.1$.

HRMS (ESI) $\mathrm{m} / \mathrm{z}$ calcd for $\mathrm{C}_{26} \mathrm{H}_{19} \mathrm{OPNa}(\mathrm{M}+\mathrm{Na})^{+}: 401.1071$, found : 401.1053 .

\section{1-Phenyl-2,3-di-( $p$-methoxyphenyl)-1H-phosphindole-1-oxide (3b)}

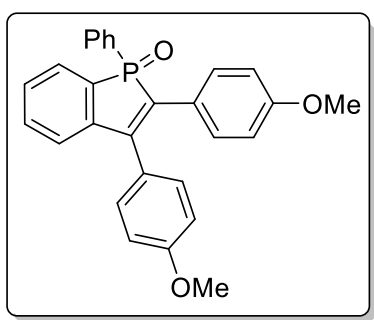

According to GP, sodium hydrogenocarbonate $(50 \mathrm{mg}, 0.60 \mathrm{mmol}$, 1.2 eq.), $N$-ethoxy-2-methylpyridinium tetrafluoroborate $(225 \mathrm{mg}, 1.0$ mmol, 2 eq.), diphenylphosphine oxide (202 mg, $1.0 \mathrm{mmol}, 2$ eq.), 1,2-bis(4-methoxyphenyl)ethyne (119 mg, $0.50 \mathrm{mmol}, 1$ eq.) and eosin Y (13 mg, $0.020 \mathrm{mmol}, 0.04$ eq.) were dissolved in DMF and irradiated during $48 \mathrm{~h}$. Purification by flash chromatography (silica gel, $n$-pentane/EtOAc : $3 / 7$ ) afforded the benzophosphole oxide $\mathbf{3 b}$ (191 $\mathrm{mg}, 88 \%$ ) as a yellow solid.

${ }^{1} \mathrm{H} \mathrm{NMR}\left(400 \mathrm{MHz}, \mathrm{CDCl}_{3}\right): \delta=3.71(\mathrm{~s}, 3 \mathrm{H}), 3.88$ (s, 3H), 6.67 (d, $\left.J=8.8 \mathrm{~Hz}, 2 \mathrm{H}\right), 7.00$ (d, $J$ $=8.7 \mathrm{~Hz}, 2 \mathrm{H}), 7.24-7.32(\mathrm{~m}, 5 \mathrm{H}), 7.33-7.38(\mathrm{~m}, 1 \mathrm{H}), 7.39-7.50(\mathrm{~m}, 4 \mathrm{H}), 7.71(\mathrm{dd}, J=7.3, J=$ $9.7 \mathrm{~Hz}, 1 \mathrm{H}), 7.77-7.82(\mathrm{~m}, 2 \mathrm{H})$.

${ }^{13} \mathrm{C}\left\{{ }^{1} \mathrm{H}\right\} \mathrm{NMR}\left(100 \mathrm{MHz}, \mathrm{CDCl}_{3}\right) \delta=55.2,55.4,113.9,114.6,123.8(\mathrm{~d}, J=11.4 \mathrm{~Hz}), 125.4$ (d, $J=10.3 \mathrm{~Hz}), 126.7(\mathrm{~d}, J=16.0 \mathrm{~Hz}), 128.7$ (d, $J=10.3 \mathrm{~Hz}), 128.8(\mathrm{~d}, J=11.4 \mathrm{~Hz}), 129.0$ $(\mathrm{d}, J=9.2 \mathrm{~Hz}), 130.4(\mathrm{~d}, J=98.4 \mathrm{~Hz}), 130.5(\mathrm{~d}, J=5.7 \mathrm{~Hz}), 130.6,131.0(\mathrm{~d}, J=9.6 \mathrm{~Hz}), 132.1$ (d, $J=104.1 \mathrm{~Hz}), 132.2(\mathrm{~d}, J=2.3 \mathrm{~Hz}), 132.9$ (d, $J=2.3 \mathrm{~Hz}), 133.2(\mathrm{~d}, J=96.1 \mathrm{~Hz}), 144.3$ (d, $J=27.5 \mathrm{~Hz}), 148.3(\mathrm{~d}, J=21.7 \mathrm{~Hz}), 159.2,159.9$.

${ }^{31} \mathrm{P}\left\{{ }^{1} \mathrm{H}\right\} \mathrm{NMR}\left(\mathrm{CDCl}_{3}, 162 \mathrm{MHz}\right): \delta=39.1$.

HRMS (ESI) m/z calcd for $\mathrm{C}_{28} \mathrm{H}_{23} \mathrm{O}_{3} \mathrm{PNa}(\mathrm{M}+\mathrm{Na})^{+}$: 461.1283, found : 461.1275 . 


\section{1-Phenyl-2,3-di-( $p$-trifluoromethylphenyl)-1H-phosphindole-1-oxide (3c) :}

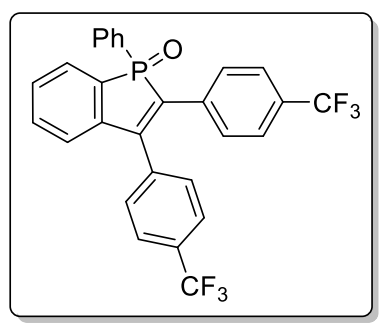

According to GP, sodium hydrogenocarbonate $(50 \mathrm{mg}, 0.60 \mathrm{mmol}$, 1.2 eq.), $N$-ethoxy-2-methylpyridinium tetrafluoroborate $(225 \mathrm{mg}, 1.0$ mmol, 2 eq.), diphenylphosphine oxide (202 mg, $1.0 \mathrm{mmol}, 2$ eq.), 1,2-bis(4-trifluoromethylphenyl)ethyne (119 mg, $0.50 \mathrm{mmol}, 1$ eq.) and eosin Y (13 mg, $0.02 \mathrm{mmol}, 0.04$ eq.) were dissolved in DMF and irradiated for $48 \mathrm{~h}$. Purification by flash chromatography (silica gel, n-pentane/EtOAc : 6/4) afforded the benzophosphole oxide $3 \mathbf{c}(126 \mathrm{mg}, 64 \%)$ as a colourless solid.

${ }^{1} \mathrm{H}$ NMR (400 MHz, $\left.\mathrm{CDCl}_{3}\right): \delta=7.17(\mathrm{dd}, J=2.8 \mathrm{~Hz}, J=7.6 \mathrm{~Hz}, 1 \mathrm{H}), 7.29-7.33(\mathrm{~m}, 2 \mathrm{H}), 7.35-$ $7.46(\mathrm{~m}, 7 \mathrm{H}), 7.49-7.55(\mathrm{~m}, 2 \mathrm{H}), 7.70-7.76(\mathrm{~m}, 5 \mathrm{H})$.

${ }^{13} \mathrm{C}\left\{{ }^{1} \mathrm{H}\right\} \mathrm{NMR}\left(100 \mathrm{MHz}, \mathrm{CDCl}_{3}\right) \delta=122.5,124.3(\mathrm{~d}, J=15.4 \mathrm{~Hz}), 125.3,125.5,126.3,128.4$, $128.9(\mathrm{~d}, J=100.2 \mathrm{~Hz}), 129.2(\mathrm{~d}, J=5.7 \mathrm{~Hz}), 129.4(\mathrm{~d}, J=30.9 \mathrm{~Hz}), 129.5(\mathrm{~d}, J=10.4 \mathrm{~Hz})$, $130.1(\mathrm{~d}, J=16.5 \mathrm{~Hz}), 131.0(\mathrm{~d}, J=16.5 \mathrm{~Hz}), 131.4(\mathrm{~d}, J=12.8 \mathrm{~Hz}), 132.4,132.8$ (d, $J=4.4$ $\mathrm{Hz}), 133.4(\mathrm{~d}, J=3.0 \mathrm{~Hz}), 134.7(\mathrm{~d}, J=94.1 \mathrm{~Hz}), 135.1,136.0(\mathrm{~d}, J=16.5 \mathrm{~Hz}), 137.5$ (d, $J=$ 21.0 Hz), $142.8(\mathrm{~d}, J=27.3 \mathrm{~Hz}), 150.0(\mathrm{~d}, J=31.5 \mathrm{~Hz})$.

${ }^{31} \mathrm{P}\left\{{ }^{1} \mathrm{H}\right\} \mathrm{NMR}\left(\mathrm{CDCl}_{3}, 162 \mathrm{MHz}\right): \delta=38.6$.

${ }^{19} \mathrm{~F} \mathrm{NMR}\left(\mathrm{CDCl}_{3}, 376.5 \mathrm{MHz}\right): \delta=-62.9,-62.7$.

HRMS (ESI) m/z calcd for $\mathrm{C}_{28} \mathrm{H}_{18} \mathrm{~F}_{6} \mathrm{OP}(\mathrm{M}+\mathrm{H})^{+}: 515.0999$, found : 515.1002 .

\section{1-Phenyl-2,3-di-(fluorophenyl)-1H-phosphindole-1-oxide (3d)}

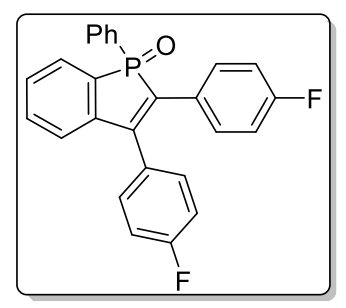

According to GP, sodium hydrogenocarbonate $(50 \mathrm{mg}, 0.60 \mathrm{mmol}, 1.2$ eq.), $N$-ethoxy-2-methylpyridinium tetrafluoroborate $(225 \mathrm{mg}, 1.0 \mathrm{mmol}$, 2 eq.), diphenylphosphine oxide (202 mg, $1.0 \mathrm{mmol}, 2$ eq.), 1,2-bis(4fluorophenyl)ethyne (107 mg, $0.50 \mathrm{mmol}, 1$ eq.) and Eosin Y (13 mg, $0.020 \mathrm{mmol}, 0.04$ eq.) were dissolved in DMF and irradiated during $48 \mathrm{~h}$.

Purification by flash chromatography (silica gel, $n$-pentane/EtOAc : 6/4) afforded the benzophosphole oxide 3d (159 mg, 77\%) as a colourless solid.

${ }^{1} \mathrm{H}$ NMR $\left(400 \mathrm{MHz}, \mathrm{CDCl}_{3}\right): \delta=6.80(\mathrm{t}, J=8.4 \mathrm{~Hz}, 2 \mathrm{H}), 7.14(\mathrm{t}, J=8.8 \mathrm{~Hz}, 2 \mathrm{H}), 7.17-7.22$ (m, 3H), 7.27-7.31 (m, 2H), 7.36-7.42 (m, 3H), 7.45-7.51 (m, 2H), 7.78-7.68 (m, 3H). 
${ }^{13} \mathrm{C}\left\{{ }^{1} \mathrm{H}\right\} \mathrm{NMR}\left(100 \mathrm{MHz}, \mathrm{CDCl}_{3}\right): \delta=115.6(\mathrm{~d}, J=22.4 \mathrm{~Hz}), 116.4(\mathrm{~d}, J=21.4 \mathrm{~Hz}), 124.0(\mathrm{~d}$, $J=10.8 \mathrm{~Hz}), 128.6(\mathrm{dd}, J=3.1 \mathrm{~Hz}, J=9.7 \mathrm{~Hz}), 129.1(\mathrm{~d}, J=12.3 \mathrm{~Hz}), 129.4(\mathrm{~d}, J=8.6 \mathrm{~Hz})$, $129.5(\mathrm{~d}, J=9.3 \mathrm{~Hz}), 129.6(\mathrm{~d}, J=99.7 \mathrm{~Hz}), 130.87$ (d, $J=5.5 \mathrm{~Hz}), 130.92 \mathrm{~Hz}(\mathrm{~d}, J=3.4 \mathrm{~Hz})$, $131.0(\mathrm{~d}, J=2.9 \mathrm{~Hz}), 131.08(\mathrm{~d}, J=1.7 \mathrm{~Hz}), 131.13(\mathrm{~d}, J=4.9 \mathrm{~Hz}), 131.8(\mathrm{~d}, J=105.4 \mathrm{~Hz})$, $132.4(\mathrm{~d}, J=2.8 \mathrm{~Hz}), 133.2(\mathrm{~d}, J=1.8 \mathrm{~Hz}), 143.5(\mathrm{~d}, J=26.4 \mathrm{~Hz}), 148.9$ (d, J = 22.3 Hz), 161.4 $(\mathrm{d}, J=57.1 \mathrm{~Hz}), 163.9(\mathrm{~d}, J=57.7 \mathrm{~Hz})$.

${ }^{31} \mathrm{P}\left\{{ }^{1} \mathrm{H}\right\}$ NMR $\left(162 \mathrm{MHz}, \mathrm{CDCl}_{3}\right): \delta=38.9$.

${ }^{19} \mathrm{~F} \mathrm{NMR}\left(\mathrm{CDCl}_{3}, 376.5 \mathrm{MHz}\right): \delta=-112.5,-111.6$.

HRMS (ESI) $\mathrm{m} / \mathrm{z}$ calcd for $\mathrm{C}_{26} \mathrm{H}_{18} \mathrm{~F}_{2} \mathrm{OP}(\mathrm{M}+\mathrm{H})^{+}: 415.1063$, found : 415.1068

\section{1-Phenyl-2,3-di-(chlorophenyl)-1H-phosphindole-1-oxide (3e)}

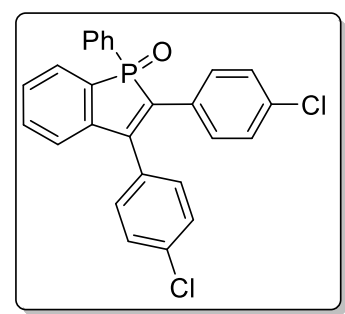

Purification by flash chromatography (silica gel, n-pentane/EtOAc : 6/4) afforded the benzophosphole oxide 3e (164 mg, 73\%) as a colourless solid.

${ }^{1} \mathrm{H}$ NMR (400 MHz, $\left.\mathrm{CDCl}_{3}\right): \delta=7.10-7.12(\mathrm{~m}, 2 \mathrm{H}), 7.18-7.23(\mathrm{~m}, 3 \mathrm{H}), 7.27-7.30(\mathrm{~m}, 2 \mathrm{H})$, 7.39-7.46 (m, 5H), 7.48-7.53 (m, 2H), 7.72-7.77 (m, 3H).

${ }^{13} \mathrm{C}\left\{{ }^{1} \mathrm{H}\right\}$ NMR $\left(100 \mathrm{MHz}, \mathrm{CDCl}_{3}\right): \delta=124.0(\mathrm{~d}, J=10.8 \mathrm{~Hz}), 128.8,129.1(\mathrm{~d}, J=20.4 \mathrm{~Hz})$, $129.4(\mathrm{~d}, J=9.6 \mathrm{~Hz}), 129.59,129.62(\mathrm{~d}, J=8.8 \mathrm{~Hz}), 129.7$ (d, $J=23.3 \mathrm{~Hz}), 130.3$ (d, $J=5.5$ Hz), 130.5, 130.91 (d, $J=10.5 \mathrm{~Hz}), 130.94$ (d, $J=10.6 \mathrm{~Hz}), 131.7$ (d, $J=94.1 \mathrm{~Hz}), 132.4$ (d, $J=3.6 \mathrm{~Hz}), 132.5(\mathrm{~d}, J=2.8 \mathrm{~Hz}), 133.2(\mathrm{~d}, J=1.7 \mathrm{~Hz}), 134.0(\mathrm{~d}, J=94.7 \mathrm{~Hz}), 134.1,135.0$, $143.2(\mathrm{~d}, J=26.5 \mathrm{~Hz}), 149.1(\mathrm{~d}, J=21.5 \mathrm{~Hz})$.

${ }^{31} \mathrm{P}\left\{{ }^{1} \mathrm{H}\right\}$ NMR $\left(\mathrm{CDCl}_{3}, 162 \mathrm{MHz}\right): \delta=38.9$.

HRMS (ESI) m/z calcd for $\mathrm{C}_{26} \mathrm{H}_{17} \mathrm{Cl}_{2} \mathrm{OPNa}(\mathrm{M}+\mathrm{Na})^{+}: 469.0292$, found : 469.0278 .

\section{1,3-Diphenyl-2-methyl-1H-phosphindole-1-oxide (3f)}




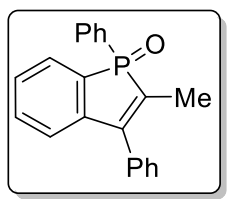

According to GP, sodium hydrogenocarbonate (50 mg, $0.60 \mathrm{mmol}, 1.2 \mathrm{eq}$.), $N$ ethoxy-2-methylpyridinium tetrafluoroborate (225 mg, $1.0 \mathrm{mmol}, 2$ eq.), diphenylphosphine oxide (202 mg, $1.0 \mathrm{mmol}, 2$ eq.), 1-phenylprop-1-yne (58 $\mathrm{mg}, 0.50 \mathrm{mmol}, 1$ eq.) and eosin Y (13 mg, $0.02 \mathrm{mmol}, 0.04$ eq.) were dissolved in DMF and irradiated during $48 \mathrm{~h}$. Purification by flash chromatography (silica gel, $n$-pentane/EtOAc : 7/3) afforded the benzophosphole oxide $\mathbf{3 f}$ (101 mg, 64\%) as a colourless solid.

${ }^{1} \mathrm{H} \mathrm{NMR}\left(500 \mathrm{MHz}, \mathrm{CDCl}_{3}\right): \delta=1.90(\mathrm{~d}, J=12.3 \mathrm{~Hz}, 3 \mathrm{H}), 7.11(\mathrm{dd}, J=2.4 \mathrm{~Hz}, J=7.6 \mathrm{~Hz}$, $1 \mathrm{H}), 7.29-7.36(\mathrm{~m}, 3 \mathrm{H}), 7.39-7.54(\mathrm{~m}, 7 \mathrm{H}), 7.66(\mathrm{dd}, J=7.0, J=9.4 \mathrm{~Hz}, 1 \mathrm{H}), 7.75(\mathrm{dd}, J=7.6$ $\mathrm{Hz}, J=12.0 \mathrm{~Hz}, 2 \mathrm{H})$.

${ }^{13} \mathrm{C}\left\{{ }^{1} \mathrm{H}\right\} \mathrm{NMR}\left(150 \mathrm{MHz}, \mathrm{CDCl}_{3}\right): \delta=10.8(\mathrm{~d}, J=10.6 \mathrm{~Hz}), 123.3(\mathrm{~d}, J=10.9 \mathrm{~Hz}), 128.5(\mathrm{~d}$, $J=10.3 \mathrm{~Hz}), 128.7,128.8,128.9,129.0,129.1,129.2,129.5(\mathrm{~d}, J=78.1 \mathrm{~Hz}) 131.1(\mathrm{~d}, J=10.5$ $\mathrm{Hz}), 131.4(\mathrm{~d}, J=58.9 \mathrm{~Hz}), 132.3(\mathrm{~d}, J=40.9 \mathrm{~Hz}), 132.4(\mathrm{~d}, J=2.7 \mathrm{~Hz}), 133.0(\mathrm{~d}, J=2.3 \mathrm{~Hz})$, $133.7(\mathrm{~d}, J=16.3 \mathrm{~Hz}), 144.4(\mathrm{~d}, J=28.3 \mathrm{~Hz}), 150.1(\mathrm{~d}, J=21.8 \mathrm{~Hz})$.

${ }^{31} \mathrm{P}\left\{{ }^{1} \mathrm{H}\right\} \mathrm{NMR}\left(\mathrm{CDCl}_{3}, 162 \mathrm{MHz}\right): \delta=40.4$.

HRMS (ESI) $\mathrm{m} / \mathrm{z}$ calcd for $\mathrm{C}_{21} \mathrm{H}_{17} \mathrm{OPNa}(\mathrm{M}+\mathrm{Na})^{+}: 339.0915$, found : 339.0908 .

\section{1,3-Diphenyl-2-ethyl-1H-phosphindole-1-oxide (3g) :}

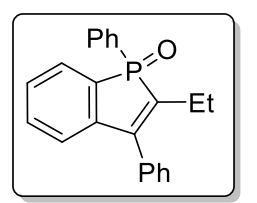

According to GP, sodium hydrogenocarbonate (50 mg, $0.60 \mathrm{mmol}, 1.2 \mathrm{eq}$.$) ,$ $N$-ethoxy-2-methylpyridinium tetrafluoroborate (225 mg, $1.0 \mathrm{mmol}, 2$ eq.), diphenylphosphine oxide (202 mg, 1.0 mmol, 2 eq.), 1-phenylbut-1-yne (65 $\mathrm{mg}, 0.50 \mathrm{mmol}, 1$ eq.) and eosin Y (13 mg, $0.02 \mathrm{mmol}, 0.04$ eq.) were dissolved in DMF and irradiated during 48h. Purification by flash chromatography (silica gel, n-pentane/EtOAc : 6/4) afforded the benzophosphole oxide $\mathbf{3 g}(150 \mathrm{mg}, 91 \%)$ as a colourless solid.

${ }^{1} \mathrm{H}$ NMR (400 MHz, $\left.\mathrm{CDCl}_{3}\right): \delta=0.95(\mathrm{t}, J=7.9 \mathrm{~Hz}), 2.19-2.31(\mathrm{~m}, 1 \mathrm{H}), 2.39-2.52(\mathrm{~m}, 1 \mathrm{H})$, $7.03(\mathrm{dd}, J=2.8 \mathrm{~Hz}, J=7.2 \mathrm{~Hz}, 1 \mathrm{H}), 7.27-7.35(\mathrm{~m}, 3 \mathrm{H}), 7.37-7.53(\mathrm{~m}, 7 \mathrm{H}), 7.58-7.64(\mathrm{~m}, 1 \mathrm{H})$, $7.76(\mathrm{dd}, J=7.2 \mathrm{~Hz}, J=12.4 \mathrm{~Hz}, 2 \mathrm{H})$.

${ }^{13} \mathrm{C}\left\{{ }^{1} \mathrm{H}\right\} \mathrm{NMR}\left(100 \mathrm{MHz}, \mathrm{CDCl}_{3}\right): \delta=14.0(\mathrm{~d}, J=1.8 \mathrm{~Hz}), 20.1(\mathrm{~d}, J=9.9 \mathrm{~Hz}), 123.4(\mathrm{~d}, J=$ $11.2 \mathrm{~Hz}), 128.6,128.68,128.76,128.9,128.97,129.02,129.4(\mathrm{~d}, J=55.3 \mathrm{~Hz}), 131.1,(\mathrm{~d}, J=$ $10.6 \mathrm{~Hz}), 131.2(\mathrm{~d}, J=79.6 \mathrm{~Hz}), 132.2(\mathrm{~d}, J=2.8 \mathrm{~Hz}), 132.9$ (d, $J=1.8 \mathrm{~Hz}), 134.0$, (d, $J=15.9$ Hz), $138.0(\mathrm{~d}, J=93.2 \mathrm{~Hz}), 144.4(\mathrm{~d}, J=27.7 \mathrm{~Hz}), 150.2(\mathrm{~d}, J=22.1 \mathrm{~Hz})$. 
${ }^{31} \mathrm{P}\left\{{ }^{1} \mathrm{H}\right\} \mathrm{NMR}\left(\mathrm{CDCl}_{3}, 162 \mathrm{MHz}\right): \delta=39.9$.

HRMS (ESI) m/z calcd for $\mathrm{C}_{22} \mathrm{H}_{19} \mathrm{OPNa}(\mathrm{M}+\mathrm{Na})^{+}: 353.1071$, found : 353.1082 .

\section{2-Ethoxycarbonyl-1,3-diphenyl-1H-phosphindole-1-oxide (3h)}

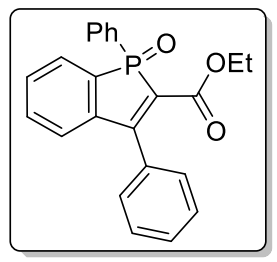

According to GP, sodium hydrogenocarbonate (50 mg, $0.60 \mathrm{mmol}, 1.2 \mathrm{eq}$.), $N$-ethoxy-2-methylpyridinium tetrafluoroborate (225 mg, $1.0 \mathrm{mmol}, 2$ eq.), diphenylphosphine oxide (202 mg, $1.0 \mathrm{mmol}, 2$ eq.), ethyl-3phenylpropiolate (87 mg, $0.50 \mathrm{mmol}, 1$ eq.) and eosin Y (13 mg, $0.02 \mathrm{mmol}$, 0.04 eq.) were dissolved in DMF and irradiated during 48h. Purification by flash chromatography (silica gel, $n$-pentane/EtOAc : 1/9) afforded the benzophosphole oxide $\mathbf{3 h}$ (142 $\mathrm{mg}, 76 \%)$ as a yellowish solid.

${ }^{1} \mathrm{H}$ NMR (400 MHz, $\left.\mathrm{CDCl}_{3}\right): \delta=0.97(\mathrm{t}, J=7.1 \mathrm{~Hz}, 3 \mathrm{H}), 3.96-4.04(\mathrm{~m}, 1 \mathrm{H}), 4.05-4.14(\mathrm{~m}$, $1 \mathrm{H})$, 7.21-7.24 (m, 1H), 7.38-7.58 (m, 10H), 7.75-7.80 (m, 3H).

${ }^{13} \mathrm{C}\left\{{ }^{1} \mathrm{H}\right\}$ NMR $\left(100 \mathrm{MHz}, \mathrm{CDCl}_{3}\right): \delta=13.7,60.9,126.4(\mathrm{~d}, J=97.7 \mathrm{~Hz}), 126.5(\mathrm{~d}, J=10.3$ $\mathrm{Hz}), 128.0,128.4,128.7$ (d, $J=12.8 \mathrm{~Hz}), 129.4,129.6$ (d, $J=9.5 \mathrm{~Hz}), 131.1$ (d, $J=10.9 \mathrm{~Hz})$, $131.8(\mathrm{~d}, J=10.8 \mathrm{~Hz}), 132.5(\mathrm{~d}, J=3.0 \mathrm{~Hz}), 132.6(\mathrm{~d}, J=99.0 \mathrm{~Hz}), 133.2(\mathrm{~d}, J=1.9 \mathrm{~Hz}), 142.4$ $(\mathrm{d}, J=25.0 \mathrm{~Hz}), 162.6(\mathrm{~d}, J=12.2 \mathrm{~Hz}), 164.0(\mathrm{~d}, J=17.9 \mathrm{~Hz})$.

${ }^{31} \mathrm{P}\left\{{ }^{1} \mathrm{H}\right\} \mathrm{NMR}\left(\mathrm{CDCl}_{3}, 162 \mathrm{MHz}\right): \delta=35.7$.

HRMS (ESI) m/z calcd for $\mathrm{C}_{23} \mathrm{H}_{20} \mathrm{O}_{3} \mathrm{P}(\mathrm{M}+\mathrm{H})^{+}: 375.1145$, found : 411.1149 .

\section{1-Phenyl-2,3-di- $n$-propyl-1H-phosphindole-1-oxide (3i)}

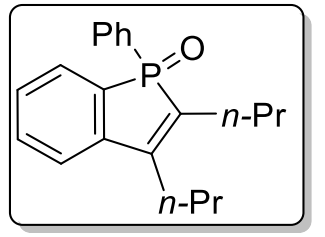

According to GP, sodium hydrogenocarbonate $(50 \mathrm{mg}, 0.60 \mathrm{mmol}, 1.2$ eq.), $N$-ethoxy-2-methylpyridinium tetrafluoroborate $(225 \mathrm{mg}, 1.0 \mathrm{mmol}$, 2 eq.), diphenylphosphine oxide (202 mg, $1.0 \mathrm{mmol}, 2$ eq.), oct-4-yne (55 $\mathrm{mg}, 0.50 \mathrm{mmol}, 1$ eq.) and eosin Y (13 mg, $0.02 \mathrm{mmol}, 0.04$ eq.) were dissolved in DMF and irradiated for 48h. Purification by flash chromatography (silica gel, $n$ pentane/EtOAc : 1/1) afforded the benzophosphole oxide $\mathbf{3 i}(126 \mathrm{mg}, 78 \%)$ as a colourless solid.

${ }^{1} \mathrm{H}$ NMR (400 MHz, $\left.\mathrm{CDCl}_{3}\right): \delta=0.85(\mathrm{t}, J=7.2 \mathrm{~Hz}, 3 \mathrm{H}), 1.06(\mathrm{t}, J=7.2 \mathrm{~Hz}, 3 \mathrm{H}), 1.38-1.48$ (m, 2H), 1.60-1.68 (m, 2H), 2.21-2.32 (m, 1H), 2.42-2.54 (m, 1H), $2.60(\mathrm{t}, J=7.6 \mathrm{~Hz}, 2 \mathrm{H})$, 7.24-7.30 (m, 1H), 7.35-7.56 (m, 7H), 7.62-7.68 (dd, $J=7.2 \mathrm{~Hz}, J=12.0 \mathrm{~Hz}, 2 \mathrm{H})$. 
${ }^{13} \mathrm{C}\left\{{ }^{1} \mathrm{H}\right\} \mathrm{NMR}\left(100 \mathrm{MHz}, \mathrm{CDCl}_{3}\right): \delta=14.4,14.5,21.9(\mathrm{~d}, J=1.6 \mathrm{~Hz}), 22.4(\mathrm{~d}, J=1.6 \mathrm{~Hz})$, $28.4(\mathrm{~d}, J=10.8 \mathrm{~Hz}), 28.6(\mathrm{~d}, J=12.1 \mathrm{~Hz}), 121.4(\mathrm{~d}, J=11.3 \mathrm{~Hz}), 128.3(\mathrm{~d}, J=10.4 \mathrm{~Hz}), 128.4$ $(\mathrm{d}, J=11.9 \mathrm{~Hz}), 128.6(\mathrm{~d}, J=9.1 \mathrm{~Hz}), 128.7(\mathrm{~d}, J=11.9 \mathrm{~Hz}), 130.4(\mathrm{~d}, J=96.5 \mathrm{~Hz}), 131.0(\mathrm{~d}$, $J=10.5 \mathrm{~Hz}), 131.7(\mathrm{~d}, J=10.0 \mathrm{~Hz}), 132.0(\mathrm{~d}, J=2.6 \mathrm{~Hz}), 132.8(\mathrm{~d}, J=1.9 \mathrm{~Hz}), 134.7$ (d, $J=$ $95.4 \mathrm{~Hz}), 143.7(\mathrm{~d}, J=29.6 \mathrm{~Hz}), 150.4(\mathrm{~d}, J=20.0 \mathrm{~Hz})$.

${ }^{31} \mathrm{P}\left\{{ }^{1} \mathrm{H}\right\} \mathrm{NMR}\left(\mathrm{CDCl}_{3}, 162 \mathrm{MHz}\right): \delta=39.8$.

HRMS (ESI) m/z calcd for $\mathrm{C}_{20} \mathrm{H}_{23} \mathrm{OPNa}(\mathrm{M}+\mathrm{Na})^{+}: 333.1384$, found : 333.1384 .

\section{1,3-Diphenyl-2-thiophenyl-1H-phosphindole-1-oxide (3j)}

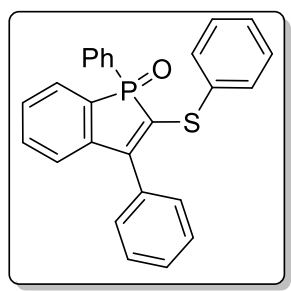

According to GP, sodium hydrogenocarbonate $(50 \mathrm{mg}, 0.60 \mathrm{mmol}, 1.2$ eq.), $N$-ethoxy-2-methylpyridinium tetrafluoroborate $(225 \mathrm{mg}, 1.0 \mathrm{mmol}$, 2 eq.), diphenylphosphine oxide (202 mg, $1.0 \mathrm{mmol}, 2$ eq.), 1-phenyl-2thiophenylet-1-yne (105 mg, $0.50 \mathrm{mmol}, 1$ eq.) and eosin Y (13 mg, 0.02 mmol, 0.04 eq.) were dissolved in DMF and irradiated during 48h. Purification by flash chromatography (silica gel, $n$-pentane/EtOAc : 6/4) afforded the benzophosphole oxide 3j (164 mg, 73\%) as a colourless gum.

${ }^{1} \mathrm{H}$ NMR (400 MHz, $\left.\mathrm{CDCl}_{3}\right): \delta=7.14-7.22(\mathrm{~m}, 3 \mathrm{H}), 7.24-7.29(\mathrm{~m}, 5 \mathrm{H})$, 7.34-7.45 (m, 3H), 7.47-7.51 (m, 2H), 7.53-7.56 (m, 2H), 7.63-7.69 (m, 3H).

${ }^{13} \mathrm{C}\left\{{ }^{1} \mathrm{H}\right\}$ NMR $\left(100 \mathrm{MHz}, \mathrm{CDCl}_{3}\right): \delta=124.6(\mathrm{~d}, J=10.6 \mathrm{~Hz}), 127.1,128.4,128.8,129.0(\mathrm{~d}, J$ = 4.4 Hz), $129.1(\mathrm{~d}, J=3.4 \mathrm{~Hz}), 129.4,129.5(\mathrm{~d}, J=11.8 \mathrm{~Hz}), 129.6,130.4(\mathrm{~d}, J=81.7 \mathrm{~Hz})$, $131.1(\mathrm{~d}, J=10.7 \mathrm{~Hz}), 131.3(\mathrm{~d}, J=105.5 \mathrm{~Hz}), 132.4(\mathrm{~d}, J=8.9 \mathrm{~Hz}), 132.5(\mathrm{~d}, J=3.0 \mathrm{~Hz})$, $133.2(\mathrm{~d}, J=2.0 \mathrm{~Hz}) 142.3(\mathrm{~d}, J=14.6 \mathrm{~Hz}), 142.4(\mathrm{~d}, J=26.3 \mathrm{~Hz})$.

${ }^{31} \mathrm{P}\left\{{ }^{1} \mathrm{H}\right\} \mathrm{NMR}\left(\mathrm{CDCl}_{3}, 162 \mathrm{MHz}\right): \delta=36.2$.

HRMS (ESI) m/z calcd for $\mathrm{C}_{26} \mathrm{H}_{20} \mathrm{OPS}(\mathrm{M}+\mathrm{H})^{+}: 411.0973$, found : 411.0970 .

\section{1-phenyl-2,3-dihydroxymethyl-1H-phosphindole-1-oxide (3k)}

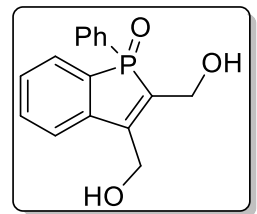

According to GP, sodium hydrogenocarbonate ( $50 \mathrm{mg}, 0.60 \mathrm{mmol}, 1.2 \mathrm{eq}$.), $N$-ethoxy-2-methylpyridinium tetrafluoroborate $(225 \mathrm{mg}, 1.0 \mathrm{mmol}, 2 \mathrm{eq}$.), diphenylphosphine oxide (202 mg, $1.0 \mathrm{mmol}, 2$ eq.), 2-butyne-1,4-diol (43 $\mathrm{mg}, 0.50 \mathrm{mmol}, 1$ eq.) and eosin Y (13 mg, $0.02 \mathrm{mmol}, 0.04$ eq.) were dissolved in DMF and irradiated for $48 \mathrm{~h}$. Purification by flash chromatography (silica gel, $n$ pentane/EtOAc : 1/1) afforded the benzophosphole oxide $\mathbf{3 k}$ (76 $\mathrm{mg}, 53 \%$ ) as a yellowish gum. 
${ }^{1} \mathrm{H} \mathrm{NMR}\left(400 \mathrm{MHz}, \mathrm{CDCl}_{3}\right): \delta=4.40(\mathrm{dd}, J=9.2 \mathrm{~Hz}, J=14.4 \mathrm{~Hz}, 1 \mathrm{H}), 4.53(\mathrm{~m}, 2 \mathrm{H}), 4.75(\mathrm{~d}$, $J=14.4 \mathrm{~Hz}, 1 \mathrm{H}), 7.30(\mathrm{dd}, J=4.0 \mathrm{~Hz}, J=7.2 \mathrm{~Hz}, 1 \mathrm{H}), 7.37-7.44(\mathrm{~m}, 4 \mathrm{H}), 7.49-7.53(\mathrm{~m}, 2 \mathrm{H})$, $7.61(\mathrm{dd}, J=7.6 \mathrm{~Hz}, J=13.2 \mathrm{~Hz}, 2 \mathrm{H})$.

${ }^{13} \mathrm{C}\left\{{ }^{1} \mathrm{H}\right\} \mathrm{NMR}\left(100 \mathrm{MHz}, \mathrm{CDCl}_{3}\right): \delta=56.1(\mathrm{~d}, J=12.0 \mathrm{~Hz}), 58.0(\mathrm{~d}, J=14.8 \mathrm{~Hz}), 123.0(\mathrm{~d}, J$ = 11.4 Hz), $128.7(\mathrm{~d}, J=26.1 \mathrm{~Hz}), 128.9(\mathrm{~d}, J=9.8 \mathrm{~Hz}), 129.1(\mathrm{~d}, J=12.6 \mathrm{~Hz}), 129.2(\mathrm{~d}, J=$ $10.2 \mathrm{~Hz}), 131.1$ (d, $J=11.1 \mathrm{~Hz}), 132.7$ (d, $J=2.5 \mathrm{~Hz}), 133.5,142.1(\mathrm{~d}, J=9.1 \mathrm{~Hz}), 152.1$ (d, $J=18.0 \mathrm{~Hz})$.

${ }^{31} \mathrm{P}\left\{{ }^{1} \mathrm{H}\right\} \mathrm{NMR}\left(\mathrm{CDCl}_{3}, 162 \mathrm{MHz}\right): \delta=42.3$.

HRMS (ESI) $\mathrm{m} / \mathrm{z}$ calcd for $\mathrm{C}_{16} \mathrm{H}_{16} \mathrm{O}_{3} \mathrm{P}(\mathrm{M}+\mathrm{H})^{+}: 287.0837$, found : 287.0833

\section{2-(diphenylphosphoryl)-1,3-diphenyl-1H-phosphindole 1-oxide (3I)}

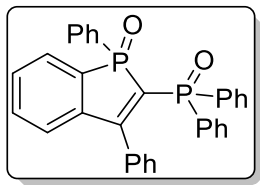

According to GP, sodium hydrogenocarbonate (50 mg, $0.60 \mathrm{mmol}, 1.2 \mathrm{eq}$.$) ,$ $N$-ethoxy-2-methylpyridinium tetrafluoroborate ( $225 \mathrm{mg}, 1.0 \mathrm{mmol}, 2$ eq.), diphenylphosphine oxide $(202 \mathrm{mg}, \quad 1.0 \mathrm{mmol}, 2 \mathrm{eq})$, diphenyl(phenylethynyl)phosphine oxide (151 mg, $0.50 \mathrm{mmol}, 1$ eq.) and eosin Y (13 mg, 0.02 mmol, 0.04 eq.) were dissolved in $\mathrm{DMF}$ and irradiated for $48 \mathrm{~h}$. Purification by flash chromatography (silica gel, $n$-pentane/EtOAc : 1/1)afforded the benzophosphole oxide 31 (208 $\mathrm{mg}, 83 \%)$ as a pale yellow solid.

${ }^{1} \mathrm{H}$ NMR $\left(\mathrm{CDCl}_{3}, 400 \mathrm{MHz}\right): \delta=6.82(\mathrm{~s}, 1 \mathrm{H}), 7.08-7.11(\mathrm{~m}, 4 \mathrm{H}), 7.18-7.22(\mathrm{~m}, 2 \mathrm{H}), 7.25-$ 7.32 (m, 4 H), 7.36-7.48 (m, 9 H), 7.54-7.57 (m, 2 H), 7.85 (dd, $J=12.5 \mathrm{~Hz}, J=7.7 \mathrm{~Hz}, 2 \mathrm{H})$. ${ }^{13} \mathrm{C}\left\{{ }^{1} \mathrm{H}\right\} \mathrm{NMR}\left(\mathrm{CDCl}_{3}, 100 \mathrm{MHz}\right): \delta=125.7(\mathrm{~d}, J=11.3 \mathrm{~Hz}), 127.8(\mathrm{~d}, J=12.5 \mathrm{~Hz}), 128.0$, $128.0(\mathrm{~d}, J=103.0 \mathrm{~Hz}), 128.5(\mathrm{dd}, J=12.7 \mathrm{~Hz}, J=20.2 \mathrm{~Hz}), 129.0(\mathrm{~d}, J=9.9 \mathrm{~Hz}), 129.2$, $129.9(\mathrm{~d}, J=13.6 \mathrm{~Hz}), 130.7$ (d, $J=11.0 \mathrm{~Hz}), 131.3(\mathrm{~d}, J=10.6 \mathrm{~Hz}), 131.6(\mathrm{~d}, J=10.2 \mathrm{~Hz})$, $131.7(\mathrm{dd}, J=2.7 \mathrm{~Hz}, J=40.2 \mathrm{~Hz}), 131.8(\mathrm{~d}, J=18.0 \mathrm{~Hz}), 132.1(\mathrm{~d}, J=10.3 \mathrm{~Hz}), 133.0,133.4$ $(\mathrm{dd}, J=5.5 \mathrm{~Hz}, J=14.3 \mathrm{~Hz}), 134.1(\mathrm{~d}, J=2.2 \mathrm{~Hz}), 134.5(\mathrm{dd}, J=5.2 \mathrm{~Hz}, J=104.1 \mathrm{~Hz}), 143.1$ $(\mathrm{dd}, J=14.3 \mathrm{~Hz}, J=27.1 \mathrm{~Hz}), 169.0(\mathrm{dd}, J=2.7 \mathrm{~Hz}, J=12.3 \mathrm{~Hz})$;

${ }^{31} \mathrm{P}\left\{{ }^{1} \mathrm{H}\right\} \mathrm{NMR}\left(\mathrm{CDCl}_{3}, 162 \mathrm{MHz}\right): \delta=17.9(\mathrm{~d}, J=36.0 \mathrm{~Hz}) ; 40.8(\mathrm{~d}, J=36.0 \mathrm{~Hz})$.

HRMS (ESI) m/z calcd for $\mathrm{C}_{32} \mathrm{H}_{25} \mathrm{O}_{2} \mathrm{P}_{2}(\mathrm{M}+\mathrm{H})^{+}:$503.1330, found : 503.1331 .

\section{1-Ethyl-2,3-diphenyl-1H-phosphindole-1-oxide (3m)}




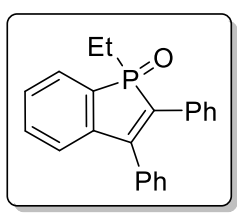

According to GP, sodium hydrogenocarbonate (50 mg, $0.60 \mathrm{mmol}, 1.2$ eq.), $\mathrm{N}$-ethoxy-2-methylpyridinium tetrafluoroborate (225 mg, $1.0 \mathrm{mmol}, 2$ eq.), ethylphenylphosphine oxide (154 mg, $1.0 \mathrm{mmol}, 2$ eq.), diphenylacetylene (89 $\mathrm{mg}, 0.50 \mathrm{mmol}, 1$ eq.) and eosin Y (13 mg, $0.02 \mathrm{mmol}, 0.04$ eq.) were dissolved in DMF and irradiated for $48 \mathrm{~h}$. Purification by flash chromatography (silica gel, $n$ pentane/EtOAc : 3/7) afforded the benzophosphole oxide $\mathbf{3 m}(110 \mathrm{mg}, 66 \%)$ as a colourless gum.

${ }^{1} \mathrm{H} \mathrm{NMR}\left(400 \mathrm{MHz}, \mathrm{CDCl}_{3}\right): \delta=1.00(\mathrm{dt}, J=19.6 \mathrm{~Hz}, J=7.6 \mathrm{~Hz}, 3 \mathrm{H}), 1.91-2.00(\mathrm{~m}, 1 \mathrm{H}) 2.10-$ $2.18(\mathrm{~d}, 1 \mathrm{H}), 7.15-7.18(\mathrm{~m}, 1 \mathrm{H}), 7.19-7.21(\mathrm{~m}, 3 \mathrm{H}), 7.27-7.29(\mathrm{~m}, 1 \mathrm{H}), 7.3-7.45(\mathrm{~m}, 7 \mathrm{H}), 7.48$ $7.53(\mathrm{~m}, 1 \mathrm{H}), 7.85(\mathrm{t}, J=6.4 \mathrm{~Hz})$.

${ }^{13} \mathrm{C}\left\{{ }^{1} \mathrm{H}\right\} \mathrm{NMR}\left(100 \mathrm{MHz}, \mathrm{CDCl}_{3}\right): \delta=6.2(\mathrm{~d}, J=3.5 \mathrm{~Hz}), 21.6(\mathrm{~d}, J=67.8 \mathrm{~Hz}), 124.0(\mathrm{~d}, J=$ $10.2 \mathrm{~Hz}), 128.0(\mathrm{~d}, J=11.7 \mathrm{~Hz}), 128.2(\mathrm{~d}, J=21.5 \mathrm{~Hz}), 128.4,128.6,128.7$ (d, $J=12.8 \mathrm{~Hz})$, $128.8(\mathrm{~d}, J=18.8 \mathrm{~Hz}), 129.0,129.1(\mathrm{~d}, J=7.8 \mathrm{~Hz}), 129.4(\mathrm{~d}, J=4.1 \mathrm{~Hz}), 130.2(\mathrm{~d}, J=99.0$ Hz), 130.3, $131.3(\mathrm{~d}, J=18.5 \mathrm{~Hz}), 132.6$ (d, $J=90.0 \mathrm{~Hz}), 132.8(\mathrm{~d}, J=1.7 \mathrm{~Hz}), 133.3$ (d, $J=$ $9.8 \mathrm{~Hz}), 134.3(\mathrm{~d}, J=14.2 \mathrm{~Hz}), 143.6(\mathrm{~d}, J=25.3 \mathrm{~Hz}), 149.3(\mathrm{~d}, J=19.9 \mathrm{~Hz})$.

${ }^{31} \mathrm{P}\left\{{ }^{1} \mathrm{H}\right\} \mathrm{NMR}\left(\mathrm{CDCl}_{3}, 162 \mathrm{MHz}\right): \delta=52.7$.

HRMS (ESI) $\mathrm{m} / \mathrm{z}$ calcd for $\mathrm{C}_{22} \mathrm{H}_{19} \mathrm{OPNa}(\mathrm{M}+\mathrm{Na})^{+}: 353.1071$, found : 353.1079 .

\section{1-tert-Butyl-2,3-diphenyl-1H-phosphindole-1-oxide (3n)}

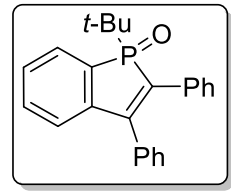

According to GP, sodium hydrogenocarbonate (50 mg, $0.60 \mathrm{mmol}, 1.2$ eq.), $N$-ethoxy-2-methylpyridinium tetrafluoroborate (225 mg, $1.0 \mathrm{mmol}, 2$ eq.), tert-butylphenylphosphine oxide (182 mg, $1.0 \mathrm{mmol}, 2$ eq. $)$, diphenylacetylene ( $89 \mathrm{mg}, 0.50 \mathrm{mmol}, 1$ eq.) and eosin Y (13 mg, $0.02 \mathrm{mmol}$, 0.04 eq.) were dissolved in DMF and irradiated for $48 \mathrm{~h}$. Purification by flash chromatography (silica gel, $n$-pentane/EtOAc : 1/4) afforded the benzophosphole oxide 3n (128 mg, 72\%) as a colourless solid.

${ }^{1} \mathrm{H}$ NMR (400MHz, $\left.\mathrm{CDCl}_{3}\right): \delta=1.08(\mathrm{~d}, J=15.2 \mathrm{~Hz}, 9 \mathrm{H}), 7.12-7.25(\mathrm{~m}, 5 \mathrm{H}), 7.33-7.45(\mathrm{~m}$, 7H), 7.79-7.83 (m, 1H).

${ }^{13} \mathrm{C}\left\{{ }^{1} \mathrm{H}\right\} \mathrm{NMR}\left(100 \mathrm{MHz}, \mathrm{CDCl}_{3}\right): \delta=24.3,32.9(\mathrm{~d}, J=67.8 \mathrm{~Hz}), 123.9(\mathrm{~d}, J=9.7 \mathrm{~Hz}), 127.7$, $128.3(\mathrm{~d}, J=12.1 \mathrm{~Hz}), 128.4(\mathrm{~d}, J=22.1 \mathrm{~Hz}), 128.8(\mathrm{br}), 129.1(\mathrm{br}), 129.2$ (d, $J=4.9 \mathrm{~Hz})$, $129.6(\mathrm{~d}, J=91.9 \mathrm{~Hz}), 129.7(\mathrm{~d}, J=8.4 \mathrm{~Hz}), 132.2(\mathrm{~d}, J=84.2 \mathrm{~Hz}), 132.5(\mathrm{~d}, J=1.5 \mathrm{~Hz}), 134.2$ $(\mathrm{d}, J=13.2 \mathrm{~Hz}), 134.6(\mathrm{~d}, J=9.4 \mathrm{~Hz}), 144.1(\mathrm{~d}, J=23.8 \mathrm{~Hz}), 150.2(\mathrm{~d}, J=18.2 \mathrm{~Hz})$. 
${ }^{31} \mathrm{P}\left\{{ }^{1} \mathrm{H}\right\} \mathrm{NMR}\left(\mathrm{CDCl}_{3}, 162 \mathrm{MHz}\right): \delta=59.3$.

HRMS (ESI) $\mathrm{m} / \mathrm{z}$ calcd for $\mathrm{C}_{24} \mathrm{H}_{24} \mathrm{OP}(\mathrm{M}+\mathrm{H})^{+}: 359.1559$, found : 359.1565 .

\section{1-Ethoxy-2,3-diphenyl-1H-phosphindole-1-oxide (3o)}

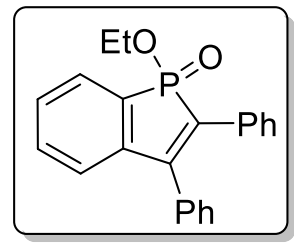

According to GP, sodium hydrogenocarbonate $(50 \mathrm{mg}, 0.60 \mathrm{mmol}, 1.2$ eq.), $N$-ethoxy-2-methylpyridinium tetrafluoroborate $(225 \mathrm{mg}, 1.0 \mathrm{mmol}$, 2 eq.), ethoxyphenylphosphine oxide (170 mg, $1.0 \mathrm{mmol}, 2$ eq.), diphenylacetylene (89 mg, $0.50 \mathrm{mmol}, 1$ eq.) and eosin Y (13 mg, 0.02 mmol, 0.04 eq.) were dissolved in $\mathrm{DMF}$ and irradiated for $48 \mathrm{~h}$. Purification by flash chromatography (silica gel, $n$-pentane/EtOAc : 1/1) afforded the benzophosphole oxide 3o (78 $\mathrm{mg}, 45 \%)$ as a colourless gum.

${ }^{1} \mathrm{H}$ NMR (400 MHz, $\left.\mathrm{CDCl}_{3}\right): \delta=1.24(\mathrm{t}, J=7.2 \mathrm{~Hz}, 3 \mathrm{H}), 4.00-4.16(\mathrm{~m}, 2 \mathrm{H}), 7.10-7.14(\mathrm{~m}$, 1H), 7.19-7.28 (m, 5H), 7.37-7.46 (m, 7H), 7.73-7.78 (m, 1H).

${ }^{13} \mathrm{C}\left\{{ }^{1} \mathrm{H}\right\} \mathrm{NMR}\left(100 \mathrm{MHz}, \mathrm{CDCl}_{3}\right): \delta=16.6(\mathrm{~d}, J=6.2 \mathrm{~Hz}), 62.3(\mathrm{~d}, J=5.9 \mathrm{~Hz}), 124.1(\mathrm{~d}, J=$ $13.4 \mathrm{~Hz}), 127.4$ (d, $J=133.1 \mathrm{~Hz}), 127.8$ (d, $J=8.9 \mathrm{~Hz}), 128.1,128.5,128.8,129.1$ (d, $J=5.1$ Hz), 129.2 (d, $J=10.2 \mathrm{~Hz}), 129.2,130.5$ (d, $J=26.4 \mathrm{~Hz}), 132.7$ (d, $J=9.0 \mathrm{~Hz}), 133.1$ (d, $J=$ $1.9 \mathrm{~Hz}), 134.0(\mathrm{~d}, J=18.0 \mathrm{~Hz}), 142.1(\mathrm{~d}, J=34.2 \mathrm{~Hz}), 148.7(\mathrm{~d}, J=27.5 \mathrm{~Hz})$.

${ }^{31} \mathrm{P}\left\{{ }^{1} \mathrm{H}\right\} \mathrm{NMR}\left(\mathrm{CDCl}_{3}, 162 \mathrm{MHz}\right): \delta=45.9$.

HRMS (ESI) $\mathrm{m} / \mathrm{z}$ calcd for $\mathrm{C}_{22} \mathrm{H}_{20} \mathrm{O}_{2} \mathrm{P}(\mathrm{M}+\mathrm{H})^{+}: 347.1195$, found : 347.1204 .

\section{5-(N,N)dimethylamine-1-[p-(N,N)dimehtylaminephenyl]-2,3-diphenyl-1H-phosphindole-}

\section{1-oxide (3p)}

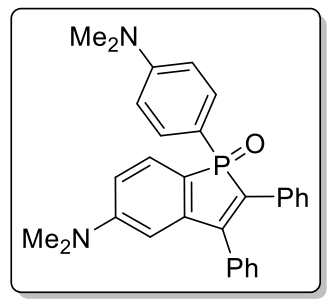

According to GP, sodium hydrogenocarbonate $(50 \mathrm{mg}, 0.60 \mathrm{mmol}, 1.2$ eq.), $N$-ethoxy-2-methylpyridinium tetrafluoroborate $(225 \mathrm{mg}, 1.0 \mathrm{mmol}$, 2 eq.), bis[4-(N,N)dimethylaminephenyl]-phosphine oxide (288 mg, 1.0 mmol, 2 eq.), diphenylacetylene ( $89 \mathrm{mg}, 0.50 \mathrm{mmol}, 1$ eq.) and eosin $\mathrm{Y}$ (13 mg, $0.02 \mathrm{mmol}, 0.04$ eq.) were dissolved in DMF and irradiated during 48 h. Purification by flash chromatography (silica gel, $n$-pentane/EtOAc : 1/1) afforded the benzophosphole oxide 3p (112 mg, 48\%) as a yellow solid. 
${ }^{1} \mathrm{H}$ NMR (400 MHz, $\left.\mathrm{CDCl}_{3}\right): \delta=2.94-2.98(\mathrm{~m}, 12 \mathrm{H}), 6.58-6.68(\mathrm{~m}, 3 \mathrm{H}), 6.99-7.09$ (m, 5H), 7.15-7.21, (m, 2H), 7.28-7.40 (m, 6H), 7.59-7.67 (m, 2H).

${ }^{13} \mathrm{C}\left\{{ }^{1} \mathrm{H}\right\}$ NMR $\left(100 \mathrm{MHz}, \mathrm{CDCl}_{3}\right): \delta=40.1,40.6,111.9(\mathrm{~d}, J=12.9 \mathrm{~Hz}), 113.0(\mathrm{~d}, J=11.3$ Hz), 114.1, 120.3, 124.0, 124.8 (d, $J=12.3 \mathrm{~Hz}), 126.9,128.1,128.2$ (d, $J=16.0 \mathrm{~Hz}), 128.8$, $129.1(\mathrm{~d}, J=5.8 \mathrm{~Hz}), 129.2,131.3(\mathrm{~d}, J=26.4 \mathrm{~Hz}), 132.5(\mathrm{~d}, J=11.9 \mathrm{~Hz}), 134.2$ (d, $J=43.9$ $\mathrm{Hz}), 135.4(\mathrm{~d}, J=7.7 \mathrm{~Hz}), 135.5,137.8,150.2$ (d, $J=23.7 \mathrm{~Hz}), 151.0(\mathrm{~d}, J=12.6 \mathrm{~Hz}), 152.7$, 157.5 .

${ }^{31} \mathrm{P}\left\{{ }^{1} \mathrm{H}\right\} \mathrm{NMR}\left(\mathrm{CDCl}_{3}, 162 \mathrm{MHz}\right): \delta=41.1$.

HRMS (ESI) m/z calcd for $\mathrm{C}_{30} \mathrm{H}_{30} \mathrm{~N}_{2} \mathrm{OP}(\mathrm{M}+\mathrm{H})^{+}:$465.2096, found : 465.2100.

\section{5-Trifluoromethyl-1-( $p$-trifluoromethylphenyl)-2,3-diphenyl-1H-phosphindole-1-oxide} (3q)

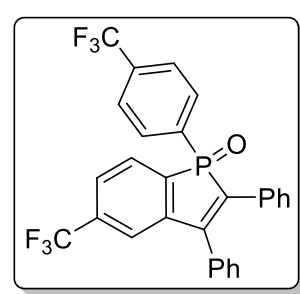

According to GP, sodium hydrogenocarbonate $(50 \mathrm{mg}, 0.60 \mathrm{mmol}, 1.2$ eq.), N-ethoxy-2-methylpyridinium tetrafluoroborate $(225 \mathrm{mg}, 1.0 \mathrm{mmol}$, 2 eq.), bis[4-trifluoromethylphenyl]-phosphine oxide (338 mg, $1.0 \mathrm{mmol}$, 2 eq.), diphenylacetylene ( $89 \mathrm{mg}, 0.50 \mathrm{mmol}, 1$ eq.) and eosin Y (13 mg, $0.02 \mathrm{mmol}, 0.04$ eq.) were dissolved in DMF and irradiated for $48 \mathrm{~h}$. Purification by flash chromatography (silica gel, $n$-pentane/EtOAc : 9/1) afforded the benzophosphole oxide 3p (178 mg, 69\%) as a colourless solid.

${ }^{1} \mathrm{H}$ NMR (400 MHz, $\left.\mathrm{CDCl}_{3}\right): \delta=7.11-7.16(\mathrm{~m}, 2 \mathrm{H}), 7.23-7.25(\mathrm{~m}, 1 \mathrm{H}), 7.31-7.39(\mathrm{~m}, 3 \mathrm{H})$, 7.45-7.49 (m, 3H), 7.66-7.71 (m, 2H), 7.73-7.76 (m, 1H), 7.87-7.97 (m, 3H).

${ }^{13} \mathrm{C}\left\{{ }^{1} \mathrm{H}\right\}$ NMR $\left(100 \mathrm{MHz}, \mathrm{CDCl}_{3}\right): \delta=124.5(\mathrm{~d}, J=10.9 \mathrm{~Hz}), 126.1(\mathrm{dq}, J=3.6 \mathrm{~Hz}, J=12.7$ $\mathrm{Hz}), 128.7,128.8,128.9,129.1(\mathrm{~d}, J=5.9 \mathrm{~Hz}), 129.4,130.7(\mathrm{q}, J=1.8 \mathrm{~Hz}) 131.3(\mathrm{~d}, J=11.1$ Hz), 131.5, 131.6, 131.7 (d, $J=2.7 \mathrm{~Hz}), 131.9$ (d, $J=14.8 \mathrm{~Hz}), 132.0$ (d, $J=5.5 \mathrm{~Hz}), 132.6$ (d, $J=10.6 \mathrm{~Hz}), 133.0,133.5(\mathrm{~d}, J=97.7 \mathrm{~Hz}), 134.5(\mathrm{dd}, J=2.9 \mathrm{~Hz}, J=22.6 \mathrm{~Hz}), 136.1(\mathrm{~d}, J=$ $96.5 \mathrm{~Hz}), 147.0(\mathrm{~d}, J=27.3 \mathrm{~Hz}), 149.6(\mathrm{~d}, J=20.6 \mathrm{~Hz})$.

${ }^{31} \mathrm{P}\left\{{ }^{1} \mathrm{H}\right\} \mathrm{NMR}\left(\mathrm{CDCl}_{3}, 162 \mathrm{MHz}\right): \delta=36.4$

${ }^{19} \mathrm{~F} \mathrm{NMR}\left(\mathrm{CDCl}_{3}, 362 \mathrm{MHz}\right): \delta=-62.7,-63.3$.

HRMS (ESI) $\mathrm{m} / \mathrm{z}$ calcd for $\mathrm{C}_{28} \mathrm{H}_{17} \mathrm{~F}_{6} \mathrm{OPNa}(\mathrm{M}+\mathrm{Na})^{+}:$:537.0819, found : 537.0806

\section{1,2,3-Triphenyl-1H-phosphindole-1-sulfide (3r)}




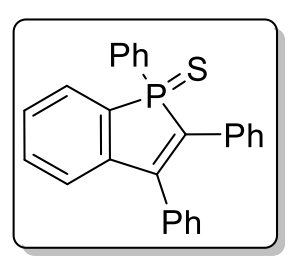

According to GP, sodium hydrogenocarbonate (50 mg, $0.60 \mathrm{mmol}, 1.2$ eq.), $\mathrm{N}$-ethoxy-2-methylpyridinium tetrafluoroborate (225 mg, $1.0 \mathrm{mmol}, 2 \mathrm{eq}$.), diphenylphosphine sulfide (218 mg, $1.0 \mathrm{mmol}, 2$ eq.), diphenylacetylene (89 mg, $0.50 \mathrm{mmol}, 1$ eq.) and eosin Y (13 mg, $0.02 \mathrm{mmol}, 0.04$ eq.) were dissolved in DMF and irradiated for $48 \mathrm{~h}$. Purification by flash chromatography (silica gel, $n$ pentane/EtOAc : 9/1) afforded the benzophosphole sulfide $3 \mathbf{r}(145 \mathrm{mg}, 74 \%)$ as a colourless solid.

${ }^{1} \mathrm{H}$ NMR (400 MHz, $\left.\mathrm{CDCl}_{3}\right): \delta=7.05-7.13(\mathrm{~m}, 3 \mathrm{H}), 7.17-7.19(\mathrm{~m}, 2 \mathrm{H}), 7.30(\mathrm{dd}, J=3.2 \mathrm{~Hz}, J$ $=7.6 \mathrm{~Hz}, 1 \mathrm{H}), 7.33-7.36(\mathrm{~m}, 2 \mathrm{H}) 7.38-7.50(\mathrm{~m}, 8 \mathrm{H}), 7.70(\mathrm{dd}, J=7.1,10.6 \mathrm{~Hz}, 1 \mathrm{H}), 7.86(\mathrm{dd}$, $J=7.0,14.1 \mathrm{~Hz}, 2 \mathrm{H})$.

${ }^{13} \mathrm{C}\left\{{ }^{1} \mathrm{H}\right\} \mathrm{NMR}\left(100 \mathrm{MHz}, \mathrm{CDCl}_{3}\right): \delta=124.5(\mathrm{~d}, J=9.8 \mathrm{~Hz}), 128.0(\mathrm{~d}, J=1.2 \mathrm{~Hz}), 128.2,128.8$ $(\mathrm{d}, J=18.2 \mathrm{~Hz}), 128.8(\mathrm{~d}, J=10.9 \mathrm{~Hz}), 128.9(\mathrm{~d}, J=12.7 \mathrm{~Hz}), 129.4,129.5(\mathrm{~d}, J=11.1 \mathrm{~Hz})$, $129.6(\mathrm{~d}, J=5.5 \mathrm{~Hz}), 129.8,131.0(\mathrm{~d}, J=11.6 \mathrm{~Hz}), 132.1$ (d, $J=3.0 \mathrm{~Hz}), 132.4(\mathrm{~d}, J=1.8 \mathrm{~Hz})$, $132.5(\mathrm{~d}, J=10.8 \mathrm{~Hz}), 134.3(\mathrm{~d}, J=14.3 \mathrm{~Hz}), 136.0(\mathrm{~d}, J=89.5 \mathrm{~Hz}), 143.8(\mathrm{~d}, J=23.4 \mathrm{~Hz})$, $149.4(\mathrm{~d}, J=18.6 \mathrm{~Hz})$.

${ }^{31} \mathrm{P}\left\{{ }^{1} \mathrm{H}\right\} \mathrm{NMR}\left(\mathrm{CDCl}_{3}, 162 \mathrm{MHz}\right): \delta=48.1$.

HRMS (ESI) $\mathrm{m} / \mathrm{z}$ calcd for $\mathrm{C}_{26} \mathrm{H}_{19} \mathrm{SPNa}(\mathrm{M}+\mathrm{Na})^{+}: 417.0843$, found : 417.0848 .

\section{1-(Napthalen-1-yl) -3,4-Diphenylnaphtho[2,3-b]-1H-phosphindole 1-Oxide (3s)}

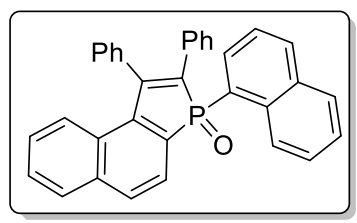

According to GP, sodium hydrogenocarbonate $(50 \mathrm{mg}, 0.60 \mathrm{mmol}, 1.2$ eq.), $N$-ethoxy-2-methylpyridinium tetrafluoroborate (225 $\mathrm{mg}, 1.0$ mmol, 2 eq.), bis[Naphtalen-2-yl]-phosphine oxide (302 mg, $1.0 \mathrm{mmol}$, 2 eq.), diphenylacetylene ( $89 \mathrm{mg}, 0.50 \mathrm{mmol}, 1$ eq.) and eosin $\mathrm{Y}$ (13 mg, 0.02 mmol, 0.04 eq.) were dissolved in DMF and irradiated for 48h. Purification by flash chromatography (silica gel, $n$-pentane/EtOAc : 6/4) afforded the benzophosphole oxide 3s (103 mg, 43\%) as a yellow solid.

${ }^{1} \mathrm{H}$ NMR (400 MHz, $\left.\mathrm{CDCl}_{3}\right): \delta=6.96-7.04(\mathrm{~m}, 2 \mathrm{H})$, 7.07-7.18 (m, 3H), 7.19-7.24 (m, 1H), 7.36-7.52 (m, 8H), $7.78(\mathrm{t}, J=8.0 \mathrm{~Hz}, 1 \mathrm{H}), 7.83-7.88(\mathrm{~m}, 2 \mathrm{H}), 8.00(\mathrm{~d}, J=8.0 \mathrm{~Hz}, 1 \mathrm{H}), 8.34$ $(\mathrm{dd}, J=7.2 \mathrm{~Hz}, J=15.6 \mathrm{~Hz}, 1 \mathrm{H}), 8.64(\mathrm{~d}, J=8.4 \mathrm{~Hz}, 1 \mathrm{H})$.

${ }^{13} \mathrm{C}\left\{{ }^{1} \mathrm{H}\right\} \mathrm{NMR}\left(100 \mathrm{MHz}, \mathrm{CDCl}_{3}\right): \delta=123.9(\mathrm{~d}, J=10.4 \mathrm{~Hz}), 125.1(\mathrm{~d}, J=13.6 \mathrm{~Hz}), 125.3(\mathrm{~d}$, $J=96.4 \mathrm{~Hz}), 125.5,125.6,126.5(\mathrm{~d}, J=21.9 \mathrm{~Hz}), 127.4(\mathrm{~d}, J=20.5 \mathrm{~Hz}), 127.7,128.1,128.8$ 
(d, $J=20.0 \mathrm{~Hz}), 129.2,129.2(\mathrm{~d}, J=29.5 \mathrm{~Hz}), 129.3(\mathrm{~d}, J=18.5 \mathrm{~Hz}), 129.6(\mathrm{~d}, J=12.2 \mathrm{~Hz})$, $130.9(\mathrm{~d}, J=10.9 \mathrm{~Hz}), 131.6(\mathrm{~d}, J=103.9), 132.9(\mathrm{~d}, J=9.7 \mathrm{~Hz}), 133.4(\mathrm{~d}, J=10.1 \mathrm{~Hz}), 133.6$ $(\mathrm{d}, J=21.1 \mathrm{~Hz}), 133.7(\mathrm{~d}, J=9.1 \mathrm{~Hz}), 137.2(\mathrm{~d}, J=77.2 \mathrm{~Hz}), 137.6(\mathrm{~d}, J=38.1 \mathrm{~Hz}), 140.0(\mathrm{~d}$, $J=26.2 \mathrm{~Hz}), 151.8(\mathrm{~d}, J=22.4 \mathrm{~Hz})$.

${ }^{31} \mathrm{P}\left\{{ }^{1} \mathrm{H}\right\} \mathrm{NMR}\left(\mathrm{CDCl}_{3}, 162 \mathrm{MHz}\right): \delta=38.6$.

HRMS (ESI) m/z calcd for $\mathrm{C}_{34} \mathrm{H}_{23} \mathrm{OPNa}(\mathrm{M}+\mathrm{Na})^{+}:$: 501.1384, found : 501.1406.

\section{1-(Napthalen-2-yl) -2,3-Diphenylnaphtho[2,3-b]-1H-phosphindole 1-Oxide (3t)}

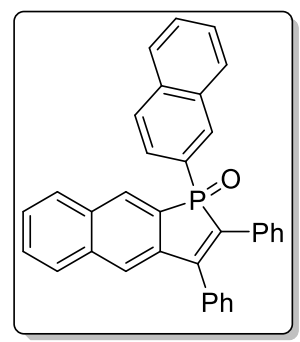

According to GP, sodium hydrogenocarbonate $(50 \mathrm{mg}, 0.60 \mathrm{mmol}, 1.2$ eq.), $\mathrm{N}$-ethoxy-2-methylpyridinium tetrafluoroborate $(225 \mathrm{mg}, 1.0 \mathrm{mmol}$, 2 eq.), bis[naphtalen-2-yl]-phosphine oxide (302 mg, $1.0 \mathrm{mmol}, 2$ eq.), diphenylacetylene (89 mg, $0.50 \mathrm{mmol}, 1$ eq.) and eosin Y (13 mg, 0.02 mmol, 0.04 eq.) were dissolved in DMF and irradiated during $48 \mathrm{~h}$. Purification by flash chromatography (silica gel, $n$-pentane/EtOAc : 6/4) afforded the benzophosphole oxide $\mathbf{3 t}(131 \mathrm{mg}$, 55\%) as a yellow solid.

${ }^{1} \mathrm{H}$ NMR (400 MHz, $\left.\mathrm{CDCl}_{3}\right): \delta=7.05-7.09$ (m, 2H), 7.27-7.30 (m, 2H), 7.38-7.50 (m, 7H), 7.52-7.56 (m, 2H), $7.73(\mathrm{dd}, J=2.4 \mathrm{~Hz}, J=8.0 \mathrm{~Hz}, 1 \mathrm{H}), 7.76-7.80(\mathrm{~m}, 1 \mathrm{H}), 7.81-7.85(\mathrm{~m}, 1 \mathrm{H})$, 7.93-8.01 (m, 2H), $8.12(\mathrm{dd}, J=2.8 \mathrm{~Hz}, J=5.2 \mathrm{~Hz}, 1 \mathrm{H}), 8.77$ (d, $J=14.0 \mathrm{~Hz}, 1 \mathrm{H})$.

${ }^{13} \mathrm{C}\left\{{ }^{1} \mathrm{H}\right\} \mathrm{NMR}\left(100 \mathrm{MHz}, \mathrm{CDCl}_{3}\right): \delta=121.5(\mathrm{~d}, J=12.2 \mathrm{~Hz}), 125.0(\mathrm{~d}, J=13.0 \mathrm{~Hz}), 125.7(\mathrm{~d}$, $J=5.0 \mathrm{~Hz}), 126.9$ (d, $J=15.9 \mathrm{~Hz}), 127.0$ (d, $J=2.7 \mathrm{~Hz}), 128.0(\mathrm{~d}, J=4.3 \mathrm{~Hz}), 128.1$ (d, $J=$ $47.9 \mathrm{~Hz}), 128.2,128.4,128.7(\mathrm{~d}, J=17.8 \mathrm{~Hz}), 128.8(\mathrm{~d}, J=13.0 \mathrm{~Hz}), 128.9(\mathrm{~d}, J=5.5 \mathrm{~Hz})$, $129.0(\mathrm{~d}, J=3.4 \mathrm{~Hz}), 129.2(\mathrm{~d}, J=5.6 \mathrm{~Hz}), 129.3(\mathrm{~d}, J=7.0 \mathrm{~Hz}), 129.5,132.0(\mathrm{~d}, J=8.8 \mathrm{~Hz})$, $132.7(\mathrm{~d}, J=10.1 \mathrm{~Hz}), 133.2(\mathrm{~d}, J=12.7 \mathrm{~Hz}), 133.7(\mathrm{~d}, J=8.3 \mathrm{~Hz}), 133.9,134.2(\mathrm{~d}, J=8.7$ $\mathrm{Hz}), 134.6$ (d, $J=39.5 \mathrm{~Hz}), 134.7$, (d, $J=15.6 \mathrm{~Hz}), 135.1$ (d, $J=2.5 \mathrm{~Hz}), 143.2$ (d, $J=26.0$ $\mathrm{Hz}), 150.0(\mathrm{~d}, J=22.6 \mathrm{~Hz}), 162.7$.

${ }^{31} \mathrm{P}\left\{{ }^{1} \mathrm{H}\right\} \mathrm{NMR}\left(\mathrm{CDCl}_{3}, 162 \mathrm{MHz}\right): \delta=39.5$.

HRMS (ESI) m/z calcd for $\mathrm{C}_{34} \mathrm{H}_{23} \mathrm{OPNa}(\mathrm{M}+\mathrm{Na})^{+}:$: 501.1406, found : 501.1404.

5-Methyl-1-p-tolyl-2,3-diphenyl-1H-phosphindole-1-oxide + 6-Methyl-1-p-tolyl-2,3diphenyl-1H-phosphindole-1-oxide (3u + 3u') 


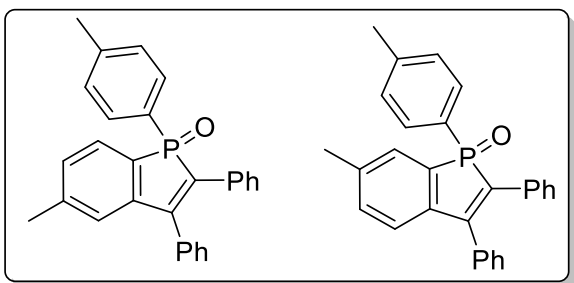

According to GP, sodium hydrogenocarbonate $(50 \mathrm{mg}$, 0.60 mmol, 1.2 eq.), N-ethoxy-2-methylpyridinium tetrafluoroborate (225 mg, $1.0 \mathrm{mmol}, 2$ eq.), bis(4tolyl)phosphine oxide (230 mg, $1.0 \mathrm{mmol}, 2$ eq.), diphenylacetylene ( $89 \mathrm{mg}, 0.50 \mathrm{mmol}, 1$ eq.) and eosin $\mathrm{Y}$

(13 mg, $0.02 \mathrm{mmol}, 0.04$ eq.) were dissolved in DMF and irradiated during 48h. Purification by flash chromatography (silica gel, $n$-pentane/EtOAc : $3 / 7)$ yielded a mixture $(1: 1)$ of the benzophosphole oxides $\mathbf{3 u}$ and 3u' (138 mg, 68\%) as a colourless solid.

${ }^{1} \mathrm{H} \mathrm{NMR}\left(400 \mathrm{MHz}, \mathrm{CDCl}_{3}\right): \delta=2.33(\mathrm{~m}, 12 \mathrm{H}), 7.01(\mathrm{~s}, 1 \mathrm{H}), 7.06-7.11(\mathrm{~m}, 6 \mathrm{H}), 7.15-7.25(\mathrm{~m}$, 10H) 7.31-7.35 (m, 4H), 7.38-7.46 (m, 6H), 7.50-7.62 (m, 3H) 7.63-7.73 (m, 4H).

${ }^{13} \mathrm{C}\left\{{ }^{1} \mathrm{H}\right\}$ NMR (100 MHz, $\left.\mathrm{CDCl}_{3}\right): \delta=21.3,21.7$ (2C, overlapped), 21.9, 123.9 (d, $J=11.5$ Hz), $124.9(\mathrm{~d}, J=11.1 \mathrm{~Hz}), 126.5$ (d, $J=101.8 \mathrm{~Hz}), 126.6$ (d, $J=101.8 \mathrm{~Hz}), 127.6,127.7$, 128.2, 128.6, 128.9, 128.9, 129.0, 129.0, 129.0, 129.1, 129.5, 129.6, 129.6, 129.7, 129.7, 129.8, $131.0(\mathrm{~d}, J=10.9 \mathrm{~Hz}$ x 2C), $132.3(\mathrm{~d}, J=86.6 \mathrm{~Hz}), 132.8(\mathrm{~d}, J=2.0 \mathrm{~Hz}), 133.0(\mathrm{~d}, J=1.9 \mathrm{~Hz})$, 133.3, 133.3, 133.7, 134.2, 134.4 (d, $J=5.7 \mathrm{~Hz}), 134.5$ (d, $J=5.4 \mathrm{~Hz}), 139.4$ (d, $J=10.3 \mathrm{~Hz})$, $141.1(\mathrm{~d}, J=26.9 \mathrm{~Hz}), 142.6(\mathrm{~d}, J=7.0 \mathrm{~Hz}), 142.7(\mathrm{~d}, J=6.4 \mathrm{~Hz}), 143.5,144.1$ (d, $J=27.2$ $\mathrm{Hz}), 149.8(\mathrm{~d}, J=21.0 \mathrm{~Hz}), 150.0(\mathrm{~d}, J=20.7 \mathrm{~Hz})$.

${ }^{31} \mathrm{P}\left\{{ }^{1} \mathrm{H}\right\} \mathrm{NMR}\left(\mathrm{CDCl}_{3}, 162 \mathrm{MHz}\right): \delta=39.3,39.6$.

HRMS (ESI) m/z calcd for $\mathrm{C}_{28} \mathrm{H}_{24} \mathrm{OP}(\mathrm{M}+\mathrm{H})^{+}: 407.1565$, found : 407.1574 .

\section{5-Methoxy-1-(p-methoxyphenyl)-2,3-diphenyl-1H-phosphindole-1-oxide (3v)}

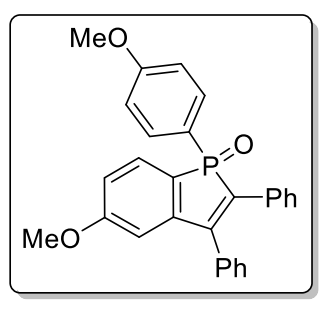

According to GP, sodium hydrogenocarbonate $(50 \mathrm{mg}, 0.60 \mathrm{mmol}, 1.2$ eq.), $N$-ethoxy-2-methylpyridinium tetrafluoroborate $(225 \mathrm{mg}, 1.0 \mathrm{mmol}$, 2 eq.), bis[4-methoxyphenyl]-phosphine oxide (262 mg, $1.0 \mathrm{mmol}, 2$ eq.), diphenylacetylene (89 mg, $0.50 \mathrm{mmol}, 1$ eq.) and eosin Y (13 mg, 0.02 mmol, 0.04 eq.) were dissolved in DMF and irradiated during 48h. benzophosphole oxide $\mathbf{3 v}$ (64 mg, 29\%) as a pale yellow solid.

${ }^{1} \mathrm{H} \mathrm{NMR}\left(400 \mathrm{MHz}, \mathrm{CDCl}_{3}\right): \delta=3.78$ (s, 3H), 3.79 (s, 3H), $6.73(\mathrm{t}, J=2.4 \mathrm{~Hz}, 1 \mathrm{H}), 6.84$ (dd, $J=2.8 \mathrm{~Hz}, J=8.0 \mathrm{~Hz}, 1 \mathrm{H}), 6.90(\mathrm{dd}, J=2.2 \mathrm{~Hz}, J=8.8 \mathrm{~Hz}, 2 \mathrm{H}), 7.07-7.10$ (m, 3H), 7.20-7.22 $(\mathrm{m}, 2 \mathrm{H}), 7.28-7.31(\mathrm{~m}, 2 \mathrm{H}), 7.38-7.42(\mathrm{~m}, 3 \mathrm{H}), 7.60-7.69(\mathrm{~m}, 3 \mathrm{H})$. 
${ }^{13} \mathrm{C}\left\{{ }^{1} \mathrm{H}\right\}$ NMR $\left(100 \mathrm{MHz}, \mathrm{CDCl}_{3}\right): \delta=55.4,55.7,111.6(\mathrm{~d}, J=11.8 \mathrm{~Hz}), 112.9(\mathrm{~d}, J=11.8$ Hz), $114.6(\mathrm{~d}, J=13.2 \mathrm{~Hz}), 121.1(\mathrm{~d}, J=106.8 \mathrm{~Hz}), 123.3(\mathrm{~d}, J=111.8 \mathrm{~Hz}), 127.9,128.3$, 128.7, 129.1, 129.1 (d, $J=5.6 \mathrm{~Hz}), 129.2,130.6(\mathrm{~d}, J=11.2 \mathrm{~Hz}), 133.0,133.1,134.4(\mathrm{~d}, J=$ $14.8 \mathrm{~Hz}), 136.3$ (d, $J=94.5 \mathrm{~Hz}), 146.3(\mathrm{~d}, J=28.2 \mathrm{~Hz}), 149.0$ (d, $J=21.2 \mathrm{~Hz}), 162.8$ (d, $J=$ $2.9 \mathrm{~Hz}), 163.8(\mathrm{~d}, J=1.9 \mathrm{~Hz})$.

${ }^{31} \mathrm{P}\left\{{ }^{1} \mathrm{H}\right\} \mathrm{NMR}\left(\mathrm{CDCl}_{3}, 162 \mathrm{MHz}\right): \delta=38.1$.

HRMS (ESI) m/z calcd for $\mathrm{C}_{28} \mathrm{H}_{24} \mathrm{O}_{3} \mathrm{P}(\mathrm{M}+\mathrm{H})^{+}:$439.1463, found : 439.1469

\section{6-Methoxy-1-(p-methoxyphenyl)-2,3-diphenyl-1H-phosphindole-1-oxide (3v')}

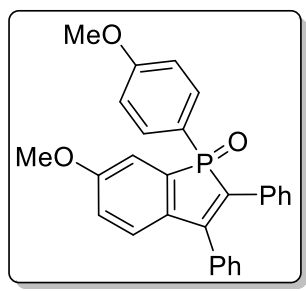

Purification by flash chromatography (silica gel, $n$-pentane/EtOAc : 7/3) afforded the benzophosphole oxide 3v' (59 mg, 27\%) as a colourless solid.

${ }^{1} \mathrm{H} \mathrm{NMR}\left(400 \mathrm{MHz}, \mathrm{CDCl}_{3}\right): \delta=3.797(\mathrm{~s}, 3 \mathrm{H}), 3.804(\mathrm{~s}, 3 \mathrm{H}), 6.91(\mathrm{dd}, J=2.0 \mathrm{~Hz}, J=8.8 \mathrm{~Hz}$, 3H), 7.07-7.13 (m, 4H), 7.18-7.20 (m, 2H), 7.25 (d, $J=10.8$ Hz, 1H), 7.30-7.32 (m, 2H), 7.39$7.42(\mathrm{~m}, 3 \mathrm{H}), 7.70(\mathrm{dd}, J=8.9 \mathrm{~Hz}, J=11.9 \mathrm{~Hz}, 2 \mathrm{H})$.

${ }^{13} \mathrm{C}\left\{{ }^{1} \mathrm{H}\right\} \operatorname{NMR}\left(100 \mathrm{MHz}, \mathrm{CDCl}_{3}\right): \delta=55.4,55.8,114.6(\mathrm{~d}, J=10.7 \mathrm{~Hz}), 114.7(\mathrm{~d}, J=13.3$ Hz), 118.0, 120.6 (d, $J=105.1 \mathrm{~Hz}), 125.3$ (d, $J=12.7 \mathrm{~Hz}), 127.6,128.3,128.7,129.0$ (d, $J=$ $8.0 \mathrm{~Hz}), 129.1$ (d, $J=8.9 \mathrm{~Hz}), 132.3$ (d, $J=97.4 \mathrm{~Hz}), 133.0$ (d, $J=11.9 \mathrm{~Hz}), 133.1$ (d, $J=10.1$ Hz), 134.5 (d, $J=104.4 \mathrm{~Hz}), 134.7$ (d, $J=15.3 \mathrm{~Hz}), 136.1$ (d, $J=26.7 \mathrm{~Hz}), 150.0$ (d, $J=21.3$ $\mathrm{Hz}), 160.8(\mathrm{~d}, J=13.4 \mathrm{~Hz}), 162.9(\mathrm{~d}, J=2.9 \mathrm{~Hz})$.

${ }^{31} \mathrm{P}\left\{{ }^{1} \mathrm{H}\right\} \mathrm{NMR}\left(\mathrm{CDCl}_{3}, 162 \mathrm{MHz}\right): \delta=39.0$.

HRMS (ESI) $\mathrm{m} / \mathrm{z}$ calcd for $\mathrm{C}_{28} \mathrm{H}_{24} \mathrm{O}_{3} \mathrm{P}(\mathrm{M}+\mathrm{H})^{+}:$: 439.1463, found : 439.1467.

\section{5-Fluoro-1-(p-fluorophenyl)-2,3-diphenyl-1H-phosphindole-1-oxide (3w)}




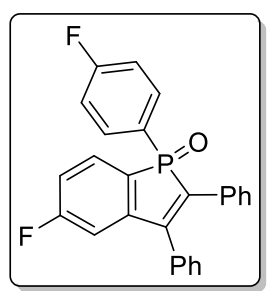

According to GP, sodium hydrogenocarbonate (50 mg, $0.60 \mathrm{mmol}, 1.2$ eq.), $N$-ethoxy-2-methylpyridinium tetrafluoroborate (225 mg, $1.0 \mathrm{mmol}, 2$ eq.), bis[4-fluorophenyl]-phosphine oxide (238 mg, $1.0 \mathrm{mmol}, 2$ eq.), diphenylacetylene ( $89 \mathrm{mg}, 0.50 \mathrm{mmol}, 1$ eq.) and eosin Y (13 mg, $0.02 \mathrm{mmol}$, 0.04 eq.) were dissolved in DMF and irradiated during 48h. Purification by flash chromatography (silica gel, $n$-pentane/EtOAc : 2/1) afforded the benzophosphole oxide 3w (108 mg, 52\%) as a white solid.

${ }^{1} \mathrm{H}$ NMR (400 MHz, $\left.\mathrm{CDCl}_{3}\right): \delta=6.89-6.95(\mathrm{~m}, 1 \mathrm{H}), 7.01-7.17(\mathrm{~m}, 6 \mathrm{H}), 7.20-7.25(\mathrm{~m}, 2 \mathrm{H})$, 7.29-7.34 (m, 2H), 7.40-7.48 (m, 3H), 7.63-7.82 (m, 3H).

${ }^{13} \mathrm{C}\left\{{ }^{1} \mathrm{H}\right\} \mathrm{NMR}\left(100 \mathrm{MHz}, \mathrm{CDCl}_{3}\right): \delta=112.3(\mathrm{dd}, J=24.1 \mathrm{~Hz}, J=11.9 \mathrm{~Hz}), 115.9(\mathrm{dd}, J=22.3$ $\mathrm{Hz}, J=11.9 \mathrm{~Hz}), 116.5(\mathrm{dd}, J=21.6 \mathrm{~Hz}, J=13.6 \mathrm{~Hz}), 125.3(\mathrm{dd}, J=3.3 \mathrm{~Hz}, J=104.2 \mathrm{~Hz})$, $127.2(\mathrm{dd}, J=3.2 \mathrm{~Hz}, J=108.9 \mathrm{~Hz}), 128.4,128.5,129.0(\mathrm{~d}, J=7.9 \mathrm{~Hz}), 129.1(\mathrm{~d}, J=16.1 \mathrm{~Hz})$, 129.3, $131.1(\mathrm{dd}, J=11.2 \mathrm{~Hz}, J=10.3 \mathrm{~Hz}), 132.2(\mathrm{~d}, J=9.3 \mathrm{~Hz}), 133.5(\mathrm{~d}, J=8.7 \mathrm{~Hz}), 133.6$ $(\mathrm{d}, J=7.9 \mathrm{~Hz}), 136.2(\mathrm{~d}, J=94.6 \mathrm{~Hz}), 147.1(\mathrm{dd}, J=8.7 \mathrm{~Hz}, J=29.4 \mathrm{~Hz}), 148.7(\mathrm{dd}, J=2.4$ $\mathrm{Hz}, J=20.6 \mathrm{~Hz}), 165.5(\mathrm{dd}, J=3.3 \mathrm{~Hz}, J=252.1 \mathrm{~Hz}), 166.4(\mathrm{dd}, J=2.1 \mathrm{~Hz}, J=251.7 \mathrm{~Hz})$. ${ }^{31} \mathrm{P}\left\{{ }^{1} \mathrm{H}\right\} \mathrm{NMR}\left(162 \mathrm{MHz}, \mathrm{CDCl}_{3}\right): \delta=35.3$.

${ }^{19} \mathrm{~F} \mathrm{NMR}\left(\mathrm{CDCl}_{3}, 376.5 \mathrm{MHz}\right): \delta=-105.8,-104.5$

HRMS (ESI) $\mathrm{m} / \mathrm{z}$ calcd for $\mathrm{C}_{26} \mathrm{H}_{18} \mathrm{~F}_{2} \mathrm{OP}(\mathrm{M}+\mathrm{H})^{+}:$415.1063, found : 415.1072 .

\section{6-Fluoro-1-(p-fluorophenyl)-2,3-diphenyl-1H-phosphindole-1-oxide (3w')}

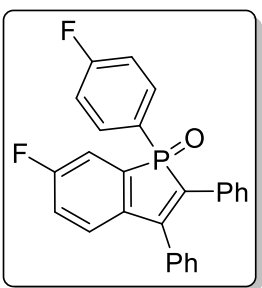

According to GP, sodium hydrogenocarbonate (50 mg, $0.60 \mathrm{mmol}, 1.2$ eq.), $\mathrm{N}$-ethoxy-2-methylpyridinium tetrafluoroborate (225 mg, $1.0 \mathrm{mmol}, 2 \mathrm{eq}$.), bis[4-fluorophenyl]-phosphine oxide (238 mg, $1.0 \mathrm{mmol}, 2$ eq.), diphenylacetylene (89 mg, $0.50 \mathrm{mmol}, 1$ eq.) and eosin Y (13 mg, 0.02 mmol, 0.04 eq.) were dissolved in DMF and irradiated for $48 \mathrm{~h}$. Purification by flash chromatography (silica gel, $n$-pentane/EtOAc : $2 / 1$ ) afforded the benzophosphole oxide 3w' (43 $\mathrm{mg}, 21 \%)$ as a white solid.

${ }^{1} \mathrm{H}$ NMR (400 MHz, $\left.\mathrm{CDCl}_{3}\right): \delta=7.06-7.15(\mathrm{~m}, 6 \mathrm{H}), 7.16-7.23(\mathrm{~m}, 3 \mathrm{H}), 7.32(\mathrm{~m}, 2 \mathrm{H}), 7.48-$ $7.36(\mathrm{~m}, 4 \mathrm{H}), 7.80-7.73(\mathrm{~m}, 2 \mathrm{H})$.

${ }^{13} \mathrm{C}\left\{{ }^{1} \mathrm{H}\right\} \mathrm{NMR}\left(100 \mathrm{MHz}, \mathrm{CDCl}_{3}\right): \delta=116.6(\mathrm{dd}, J=13.5 \mathrm{~Hz}, J=21.4 \mathrm{~Hz}), 116.8(\mathrm{dd}, J=10.5$ $\mathrm{Hz}, J=23.3 \mathrm{~Hz}), 119.7(\mathrm{~d}, J=22.8 \mathrm{~Hz}), 125.0(\mathrm{dd}, J=3.4 \mathrm{~Hz}, J=102.5 \mathrm{~Hz}), 125.9(\mathrm{dd}, J=$ $7.7 \mathrm{~Hz}, J=12.7 \mathrm{~Hz}), 128.2,128.5,129.0,129.0,129.1,129.2,132.4$ (d, $J=9.9 \mathrm{~Hz}), 133.6$ (dd, 
$J=8.9 \mathrm{~Hz}, J=12.1 \mathrm{~Hz}), 133.9(\mathrm{dd}, J=3.7 \mathrm{~Hz}, J=96.9 \mathrm{~Hz}), 134.0(\mathrm{~d}, J=15.4 \mathrm{~Hz}), 134.6(\mathrm{dd}$, $J=6.8 \mathrm{~Hz}, J=104.7 \mathrm{~Hz}), 139.5(\mathrm{dd}, J=3.0 \mathrm{~Hz}, J=27.0 \mathrm{~Hz}), 149.6(\mathrm{dd}, J=1.0 \mathrm{~Hz}, J=20.2$ $\mathrm{Hz}), 163.5(\mathrm{dd}, J=15.2 \mathrm{~Hz}, J=251.4 \mathrm{~Hz}), 165.6(\mathrm{dd}, J=3.4 \mathrm{~Hz}, J=252.4 \mathrm{~Hz})$.

${ }^{31} \mathrm{P}\left\{{ }^{1} \mathrm{H}\right\} \mathrm{NMR}\left(162 \mathrm{MHz}, \mathrm{CDCl}_{3}\right): \delta=37.0$.

${ }^{19} \mathrm{~F}$ NMR $\left(\mathrm{CDCl}_{3}, 376.5 \mathrm{MHz}\right): \delta=-110.8,-106.6$.

HRMS (ESI) m/z calcd for $\mathrm{C}_{26} \mathrm{H}_{18} \mathrm{~F}_{2} \mathrm{OP}(\mathrm{M}+\mathrm{H})^{+}$: 415.1063, found : 415.1072 .

\section{7-Methyl-2,3-diphenyl-1-(o-tolyl)-1H-phosphindole-1-oxide (3x)}

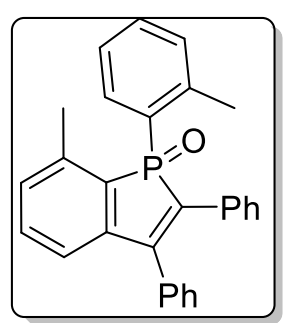

According to GP, sodium hydrogenocarbonate ( $50 \mathrm{mg}, 0.60 \mathrm{mmol}, 1.2 \mathrm{eq}$.$) ,$ $\mathrm{N}$-ethoxy-2-methylpyridinium tetrafluoroborate (225 mg, $1.0 \mathrm{mmol}, 2 \mathrm{eq}$.$) ,$ bis(2-tolyl)phosphine oxide (230 mg, $1.0 \mathrm{mmol}, 2$ eq.), diphenylacetylene (89 mg, $0.50 \mathrm{mmol}, 1$ eq.) and eosin Y (13 mg, $0.02 \mathrm{mmol}, 0.04$ eq.) were dissolved in DMF and irradiated during 48h. Purification by flash chromatography (silica gel, $n$-pentane/EtOAc : 6/4) afforded the benzophosphole oxide $\mathbf{3 w}$ (49 $\mathrm{mg}, 24 \%$ ) as a pale yellow solid.

${ }^{1} \mathrm{H}$ NMR (400 MHz, $\left.\mathrm{CDCl}_{3}\right): \delta=2.18$ (s, 3H), 2.31 (s, 3H), 7.02 (dd, $J=2.9 \mathrm{~Hz}, J=7.6 \mathrm{~Hz}$, 1H), 7.03-7.16 (m, 7H), 7.27-7.43 (m, 8H), $8.35(\mathrm{dd}, J=7.6 \mathrm{~Hz}, J=13.2 \mathrm{~Hz}, 1 \mathrm{H})$.

${ }^{13} \mathrm{C}\left\{{ }^{1} \mathrm{H}\right\} \mathrm{NMR}\left(100 \mathrm{MHz}, \mathrm{CDCl}_{3}\right): \delta=19.4(\mathrm{~d}, J=4.8 \mathrm{~Hz}), 20.0(\mathrm{~d}, J=3.8 \mathrm{~Hz}), 122.0(\mathrm{~d}, J=$ $10.5 \mathrm{~Hz}), 126.4(\mathrm{~d}, J=12.4 \mathrm{~Hz}), 128.2(\mathrm{~d}, J=84.5 \mathrm{~Hz}), .127 .9(\mathrm{~d}, J=62.3 \mathrm{~Hz}) 128.3,129.0$ $(\mathrm{d}, J=2.7 \mathrm{~Hz}), 129.0,130.8(\mathrm{~d}, J=9.5 \mathrm{~Hz}), 131.5(\mathrm{~d}, J=11.1 \mathrm{~Hz}), 132.3(\mathrm{~d}, J=2.8 \mathrm{~Hz}), 133.0$, $133.1(\mathrm{~d}, J=10.2 \mathrm{~Hz}), 133.7(\mathrm{~d}, J=104.3 \mathrm{~Hz}), 134.8(\mathrm{~d}, J=14.3 \mathrm{~Hz}), 135.0(\mathrm{~d}, J=8.5 \mathrm{~Hz})$, $135.0(\mathrm{~d}, J=8.0 \mathrm{~Hz}), 140.6(\mathrm{~d}, J=8.0 \mathrm{~Hz}), 141.2(\mathrm{~d}, J=9.5 \mathrm{~Hz}), 144.6,144.9,150.3(\mathrm{~d}, J=$ $20.6 \mathrm{~Hz})$.

${ }^{31} \mathrm{P}\left\{{ }^{1} \mathrm{H}\right\} \mathrm{NMR}\left(\mathrm{CDCl}_{3}, 162 \mathrm{MHz}\right): \delta=37.3$.

HRMS (ESI) m/z calcd for $\mathrm{C}_{28} \mathrm{H}_{24} \mathrm{OP}(\mathrm{M}+\mathrm{H})^{+}: 407.1565$, found : 407.1572 .

\section{4-Methyl-2,3-diphenyl-1-(o-tolyl)-1H-phosphindole-1-oxide (3x')}

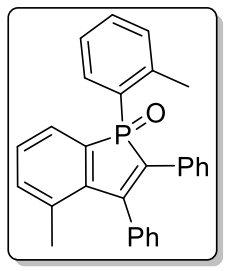

According to GP, sodium hydrogenocarbonate (50 mg, $0.60 \mathrm{mmol}, 1.2 \mathrm{eq}.), \mathrm{N}$ ethoxy-2-methylpyridinium tetrafluoroborate (225 mg, $1.0 \mathrm{mmol}, 2$ eq.), bis(2tolyl)phosphine oxide (230 mg, $1.0 \mathrm{mmol}, 2$ eq.), diphenylacetylene (89 mg, $0.50 \mathrm{mmol}, 1$ eq.) and eosin Y (13 mg, $0.020 \mathrm{mmol}, 0.04$ eq.) were dissolved in 
DMF and irradiated during 48h. Purification by flash chromatography (silica gel, $n$ pentane/EtOAc : 6/4) afforded the benzophosphole oxide 3. $\mathbf{x}^{\prime}$ (90 $\mathrm{mg}, 44 \%$ ) as a colourless solid.

${ }^{1} \mathrm{H}$ NMR (400 MHz, $\mathrm{CDCl}_{3}$ ): $\delta=1.76$ (s, 3H), 2.27 (s, 3H), 7.00-7.04 (m, 3H), 7.08-7.15 (m, 3H), 7.18- 7.26 (m, 4H), 7.30-7.42 (m, 5H), 7.50 (dd, $J=7.2 \mathrm{~Hz}, J=9.6 \mathrm{~Hz}, 1 \mathrm{H}), 8.19$ (dd, $J$ $=7.6 \mathrm{~Hz}, J=13.2 \mathrm{~Hz}, 1 \mathrm{H})$.

${ }^{13} \mathrm{C}\left\{{ }^{1} \mathrm{H}\right\} \mathrm{NMR}\left(100 \mathrm{MHz}, \mathrm{CDCl}_{3}\right): \delta=20.3(\mathrm{~d}, J=4.6 \mathrm{~Hz}), 21.5,126.2(\mathrm{~d}, J=12.1 \mathrm{~Hz}), 127.0$ $(\mathrm{d}, J=9.8 \mathrm{~Hz}), 127.6,127.7(\mathrm{~d}, J=90.2 \mathrm{~Hz}), 128.2(\mathrm{~d}, J=5.7 \mathrm{~Hz}), 128.7(\mathrm{~d}, J=3.6 \mathrm{~Hz}), 128.8$ $(\mathrm{d}, J=97.0 \mathrm{~Hz}), 128.9(\mathrm{~d}, J=5.2 \mathrm{~Hz}), 129.2(\mathrm{~d}, J=11.7 \mathrm{~Hz}), 131.5(\mathrm{~d}, J=11.2 \mathrm{~Hz}), 132.4$ (d, $J=2.8 \mathrm{~Hz}), 132.6(\mathrm{~d}, J=81.4 \mathrm{~Hz}), 133.1,134.4(\mathrm{~d}, J=9.5 \mathrm{~Hz}), 135.4(\mathrm{~d}, J=90.6 \mathrm{~Hz}), 135.8$ (d, $J=9.4 \mathrm{~Hz}), 137.4$ (d, $J=1.8 \mathrm{~Hz}), 137.7$ (d, $J=15.0 \mathrm{~Hz}), 140.9$ (d, $J=25.7 \mathrm{~Hz}), 141.3$ (d, $J=10.5 \mathrm{~Hz}), 152.5(\mathrm{~d}, J=21.3 \mathrm{~Hz})$.

${ }^{31} \mathrm{P}\left\{{ }^{1} \mathrm{H}\right\} \mathrm{NMR}\left(\mathrm{CDCl}_{3}, 162 \mathrm{MHz}\right): \delta=37.2$;

HRMS (ESI) $\mathrm{m} / \mathrm{z}$ calcd for $\mathrm{C}_{28} \mathrm{H}_{24} \mathrm{OP}(\mathrm{M}+\mathrm{H})^{+}: 407.1565$, found : 407.1555 .

\section{Mechanistic investigations}

\section{IV.1. Determination of the quantum yield of the photoreaction}

Actinometry measurements were performed with potassium Reineckate, following a method described by von Wangelin et al. ${ }^{[10]}$ Quantum-yield (QY) measurements were performed in the regime of typical organophotocatalyzed reaction (Figure S3).

The reaction was stirred for 20 minutes. During this period, the photon flux received by the reaction mixture was determined to be $3.8 \cdot 10^{-4}$ mol. ${ }^{31} \mathrm{P}$ NMR spectroscopy has been used to determine the conversion of the photocatalytic reaction of $1 \mathbf{a}$ with $\mathbf{2 a}$ by using trioctylphosphine oxide as internal standard. After 20 min of irradiation, the conversion has been estimated to be $14 \%$, which corresponds to $7.3 \cdot 10^{-5}$ mol. Based on this, the QY was calculated to be 0.19 . QY was determined as an average of at least three subsequent measurements.

\section{IV.2. EPR Spin Trapping}

\section{IV.2.1. Characterization of the ethoxy radical}


The ethoxy radical was generated upon mixing eosin $\mathrm{Y}(\mathbf{9 c})$ and $N$-ethoxy-2methylpyridinium tetrafluoroborate $\mathbf{( 8 b )}$ in tert-butylbenzene under green LEDs irradiation ( $\lambda$ $=530 \mathrm{~nm})$, and in the presence of $\alpha$-phenyl- $N$-tert-butylnitrone $(\mathrm{PBN})$ according to a procedure described elsewhere in details. ${ }^{[11]}$
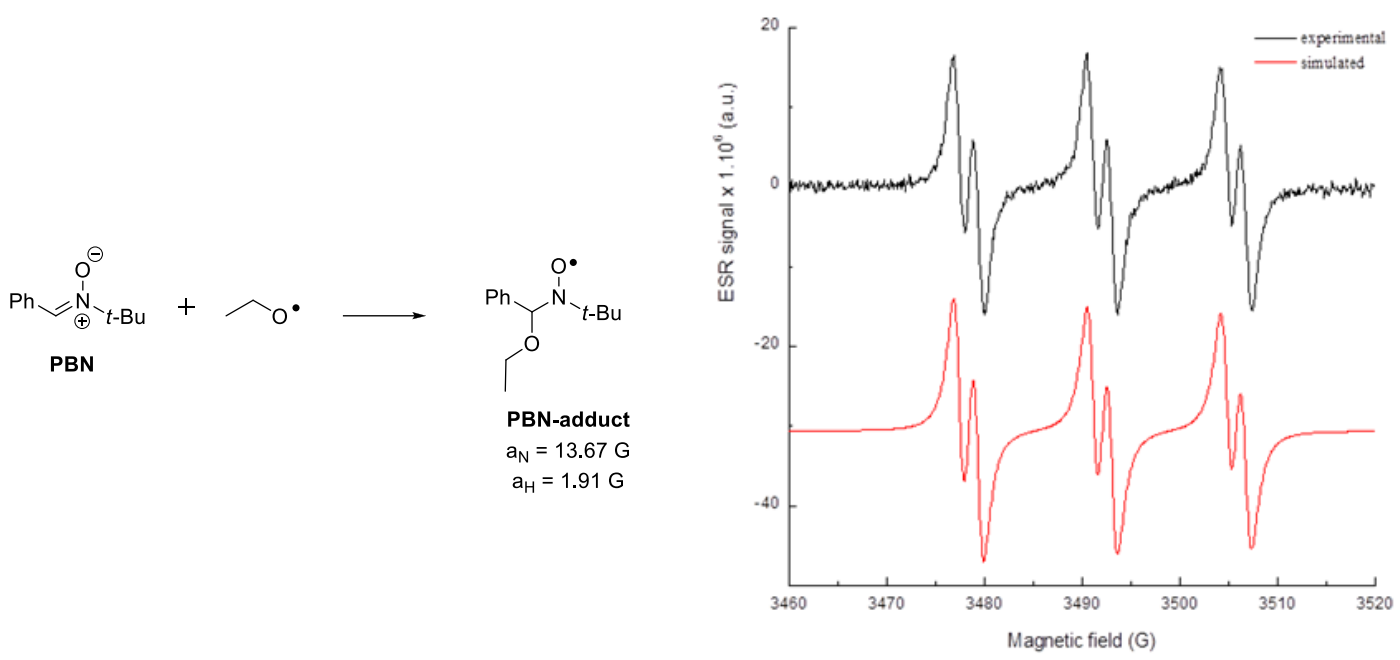

Figure S5. EPR spectra of spin adduct 11 generated in tert-butylbenzene in the presence of eosin Y $(\mathbf{9 c}) \quad\left(5 \times 10^{-5} \mathrm{M}\right), N$-ethoxy-2-methylpyridinium $8 \mathbf{b}\left(5 \times 10^{-2} \mathrm{M}\right)$ and PBN $\mathbf{1 0}$ $\left(2 \times 10^{-2} \mathrm{M}\right)$ at room temperature.

The hyperfine coupling constants determined are: $\mathrm{a}_{\mathrm{N}}=13.67 \mathrm{G}$ and $\mathrm{a}_{\mathrm{H}}=1.91 \mathrm{G}$. These values are in agreements with previously reported PBN-ethoxy adducts. ${ }^{[12]}$

\section{IV.2.2. Characterization of the phosphinoyl radical}

The title radical was generated at room temperature by mixing Eosin $\mathrm{Y}(\mathbf{9 c})$ and $\mathrm{N}$ ethoxy-2-methylpyridinium tetrafluoroborate (8b), and diphenylphosphine oxide (1a) in tertbutylbenzene under green LEDs irradiation $(\lambda=530 \mathrm{~nm})$ in the presence of $\alpha$-phenyl- $N$-tertbutylnitrone (PBN). 

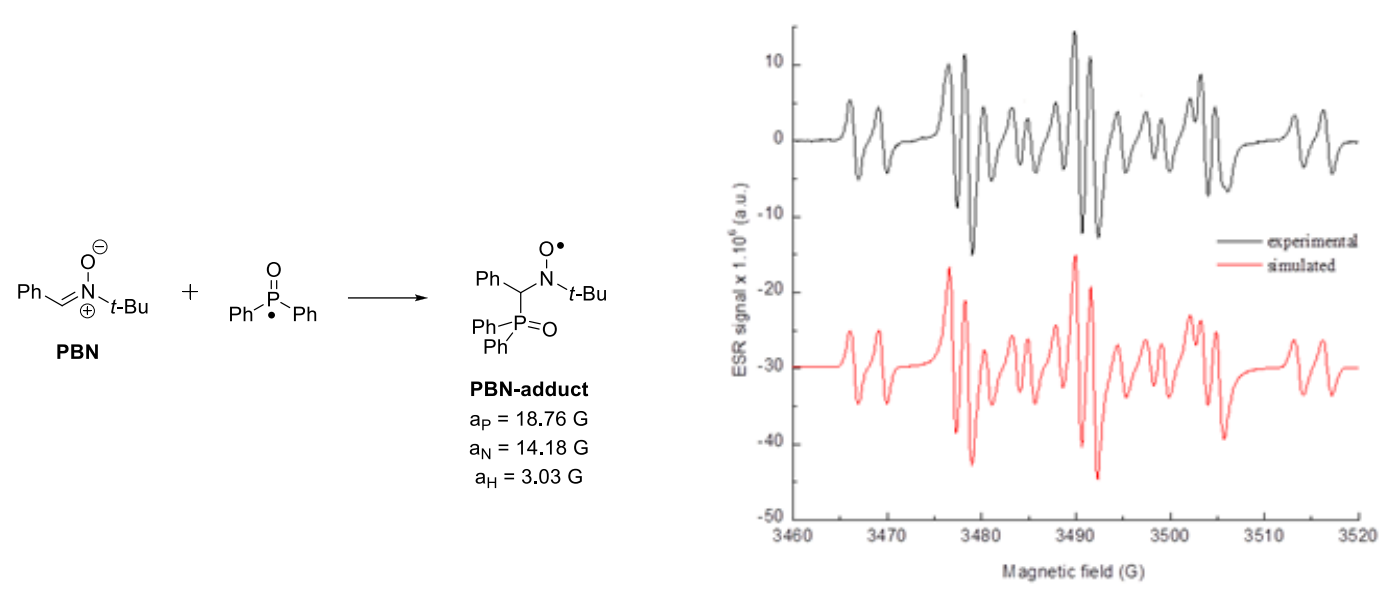

Figure S6. EPR spectra of spin adduct 12 generated in tert-butylbenzene in the presence of eosin Y $(\mathbf{9 c})\left(5 \times 10^{-5} \mathrm{M}\right), N$-ethoxy-2-methylpyridinium $\mathbf{8 b}\left(5 \times 10^{-2} \mathrm{M}\right)$, diphenylphosphine oxide $\left(2 \times 10^{-2} \mathrm{M}\right)$ and PBN $10\left(2 \times 10^{-2} \mathrm{M}\right)$ at room temperature.

The hyperfine coupling constants determined are in agreement with previously reported PBNphosphinoyl adduct. ${ }^{[13]}$

\section{IV.3. Fluorescence quenching}

The interaction rate constant $k_{\mathrm{q}}$ between Eosin $\mathrm{Y}(\mathbf{9 b})$ and the $N$-ethoxy-2methylpyridinium tetrafluoroborate $\mathbf{( 8 b}$ ), which act here as a quencher, were extracted from classical Stern-Volmer plots $\left(\mathrm{I}_{0} / \mathrm{I}=1+k q \times \tau_{0}\right.$. [quencher], where $\mathrm{I}_{0}$ and I stand for the fluorescence intensity of eosin $\mathrm{Y}$ in the absence and in the presence of the quencher, respectively; $\tau_{0}$ stands for the lifetime of the excited Eosin in the absence of quencher). 


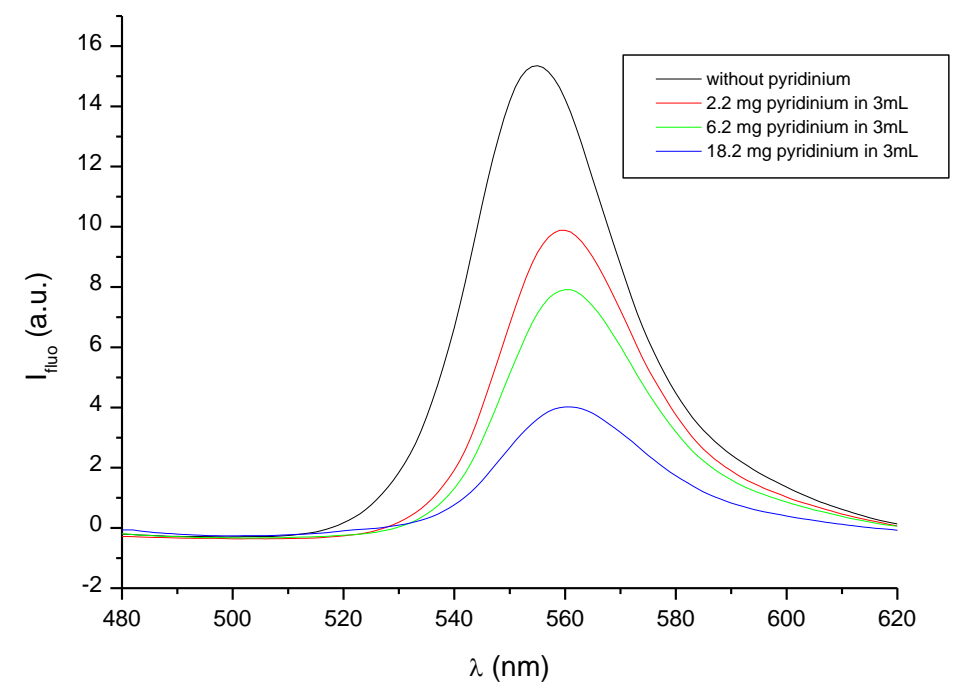

Figure S7. Fluorescence spectra of a $1.0 \times 10^{-5} \mathrm{M}$ solution of eosin $\mathrm{Y}(\mathbf{9 c})$ in DMF containing 0 (black), $3.48 \times 10^{-3} \mathrm{M}$ (red), $9.80 \times 10^{-3} \mathrm{M}$ (green), $2.88 \times 10^{-2} \mathrm{M}$ (blue) of $N$-ethoxy-2methylpyridinium tetrafluoroborate $\mathbf{( 8 b )}$.

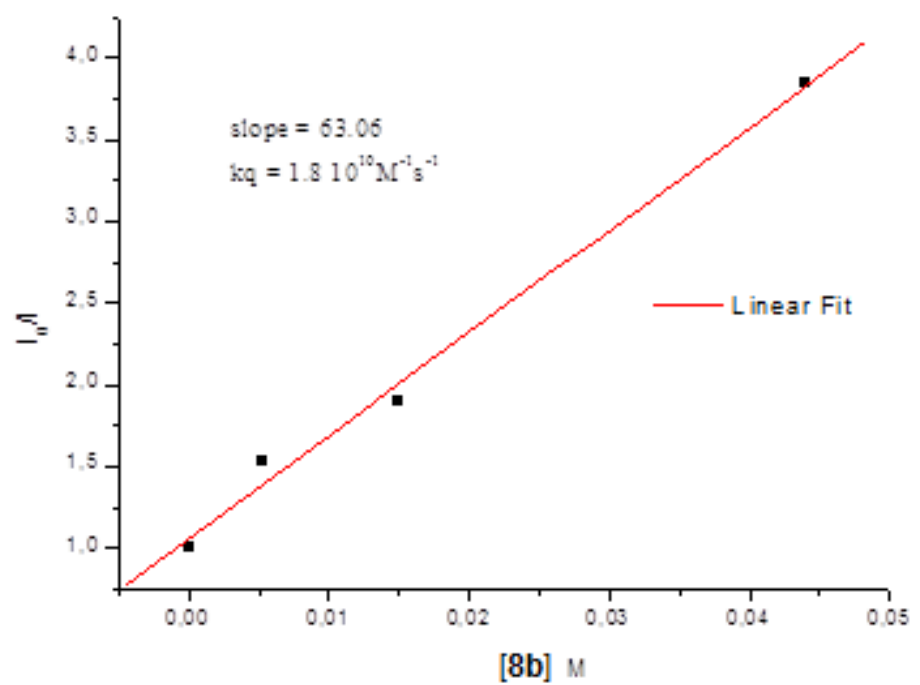

Figure S8. Stern-Volmer plots of quenching of eosin Y (9c) with N-ethoxy-2methylpyridinium tetrafluoroborate $(\mathbf{8 b})$ in DMF at $20{ }^{\circ} \mathrm{C}$.

\section{IV.4. Photolysis study}




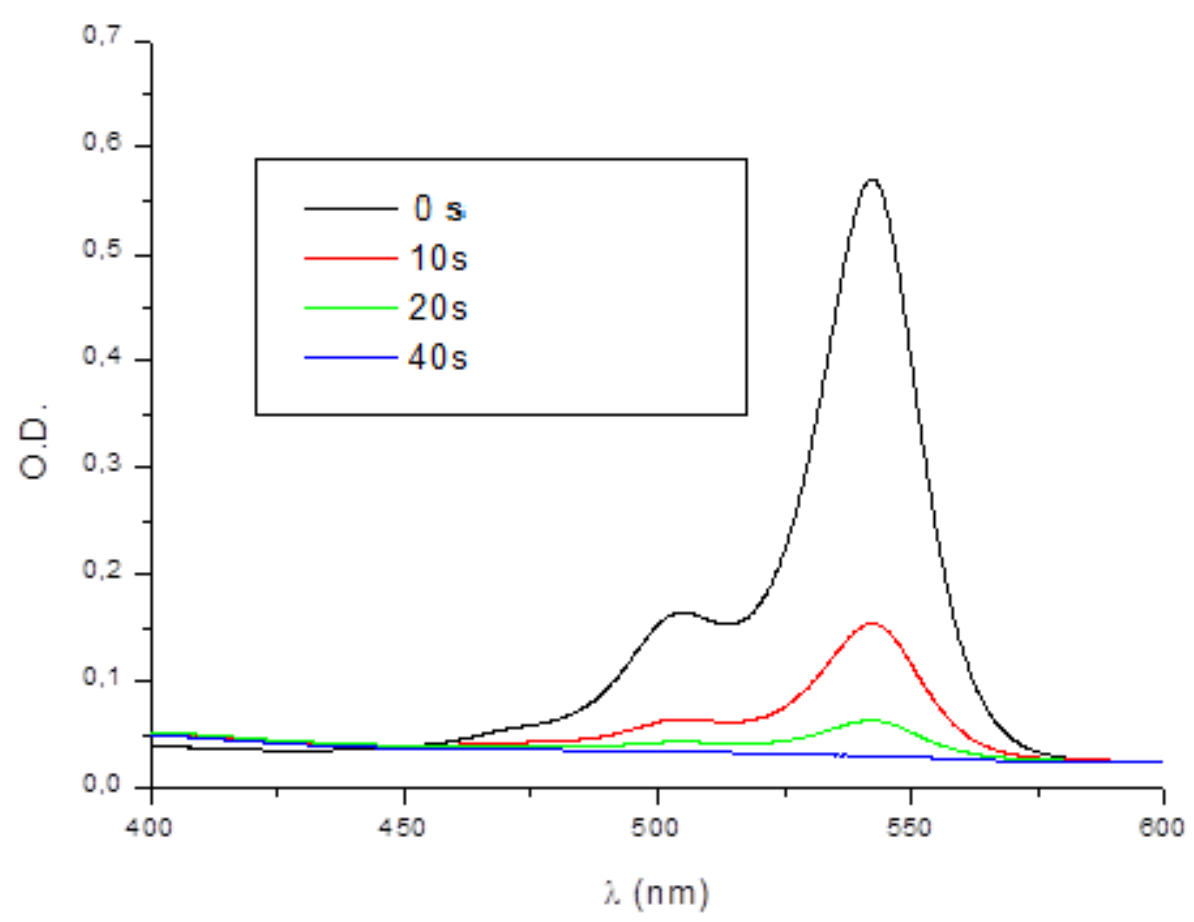

Figure S9. Changes in the UV-visible absorption spectrum of a DMF solution of EY-8b under light irradiation $\left(\lambda_{\max }=530 \mathrm{~nm}\right)$-aerated solution.

\section{X-Ray Data}

Single crystals of complex EY-8b suitable for X-ray crystallographic analysis were obtained by slow evaporation of methanol solution. X-ray diffraction experiments for monocrystal of EY-8b were performed at $150 \mathrm{~K}$ with graphite-monochromatized Mo $\mathrm{K}_{\alpha}$ radiation $(\lambda=$ $0.71073 \AA$ ) on a Bruker-Nonius Kappa CCD area detector diffractometer. Formula $\mathrm{C}_{28} \mathrm{H}_{19} \mathrm{Br}_{4} \mathrm{NO}_{6}$, formula weight 785.08, crystal system monoclinic, space group $C 2 / \mathrm{c}, a=$ 26.4265(7) $\AA, b=11.8821(3) \AA, c=19.8835(5) \AA, \beta=115.180(1)^{\circ}, \mathrm{V}=5650.2(3) \AA^{3}, \mathrm{Z}=8$, calculated density $=1.846 \mathrm{~g} / \mathrm{cm}^{3}, \mu=5.74 \mathrm{~mm}^{-1}, 38255$ measured reflections, 6477 independent reflections, $\mathrm{R}_{\text {int }}=0.0399, \mathrm{R}\left[F^{2}>2 \sigma\left(F^{2}\right)\right]=0.0556, \mathrm{w} \mathrm{R}\left(F^{2}\right)=0.1523, \mathrm{GOF}=$ 1.031, $2 \theta_{\max }=55.10^{\circ}, 320$ parameters, final difference map within 2.43 and $-2.64 \mathrm{e}^{-3}$. Program(s) used to solve structure: SHELXS-97. Program(s) used to refine structure: SHELXL-2014. Program(s) used for multi-scan absorption correction: SADABS 2012/1. CCDC 1473111 contains the supplementary crystallographic data for this paper. These data can 
be obtained free of charge from The Cambridge Crystallographic Data Center via www.ccdc.cam.ac.uk/data_request/cif.

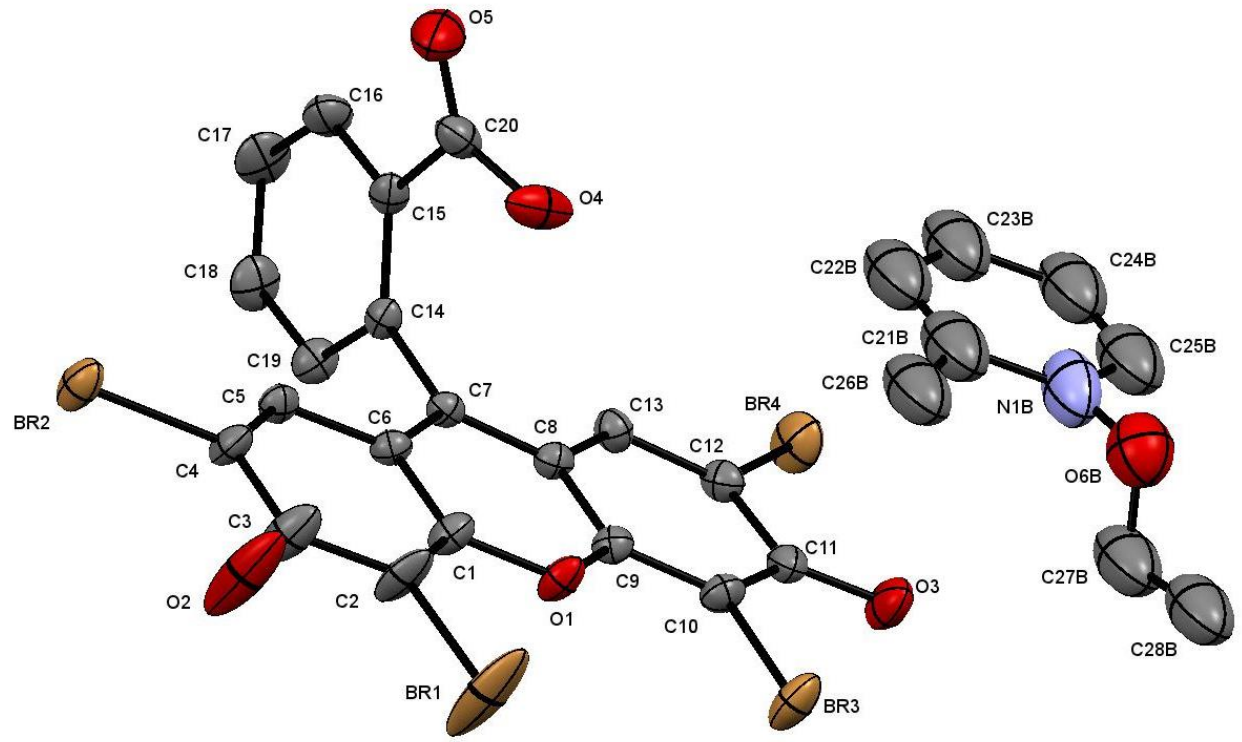

Figure S10. Asymmetric unit of EY-8b complex. Displacement ellipsoids are drawn at the 50\% probability level. For the sake of clarity, $\mathrm{H}$ atoms and disordered pyridinium moiety have been omitted.

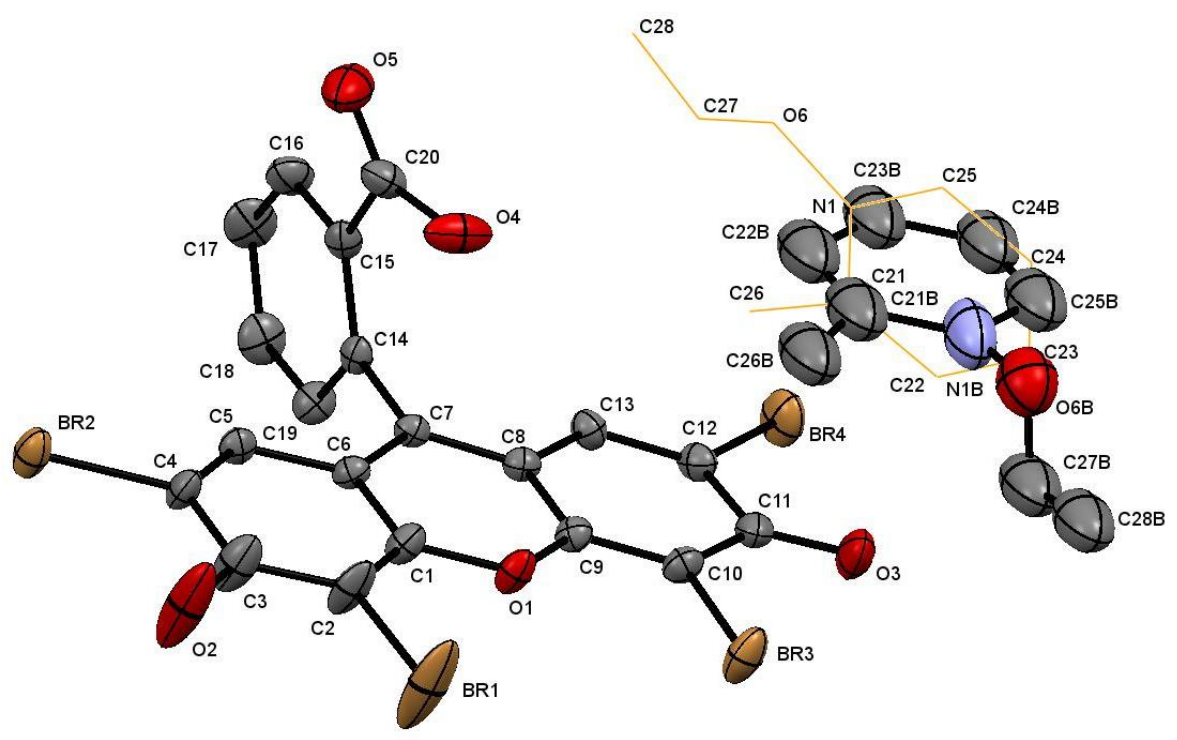

Figure S11. As indicated by the orange wireframe fragment in Figure S10, the pyridinium moiety is disordered over two positions, with an 50:50 occupancy ratio of these two 
orientations. These disordered components are allowed to refine anisotropically with implementation of rigid group and EADP constraints.

\section{References}

[1] Bielawski, M.; Olofsson, B. Org. Synth 2009, 86, 308-314.

[2] Mio, M.; Kopel, L.; Braun, J.; Gadzikwa, T.; Hull, K.; Brisbois, R.; Markworth, C.; Grieco, P. Org Lett. 2002, 4, 3199-3202

[3] Riddell, N.; Tam, W. J. Org. Chem. 2006, 71, 1934-1937.

[4] Lecercle, D. ; Sawicki, M. Org. Lett. 2006, 8, 4283-4285.

[5] Achard, T.; Giordano, L.; Tenaglia, A.; Gimbert, Y.; Buono G. Organometallics 2010, 29, $3936-3950$.

[6] Busacca, C. A.; Lorenz, J. C.; Grinberg, N.; Haddad, N.; Hrapchak, M.; Latli, B.; Lee, H.; Sabila, P.; Saha, A.; Sarvestani, M.; Shen, S.; Varsolona, R.; Wei, X.; Senanayake, C. H. Org. Lett. 2005, 7, 4277-4280.

[7] Semenzin, D.; Etemad-Moghadam, G.; Albouy, D.; Diallo, O.; Koenig, M. J. Org. Chem. 1997, 62, 2414-2422.

[8] Imazeki, S.; Sumino, M.; Fukazawa, K.; Ishihara, M.; Akiyami, T. Synthesis 2004, 10, $1648-1654$.

[9] Reichardt, C. Chem. Ber. 1966, 99, 1769-1770.

[10] Majek, M.; Filace, F.; von Wangelin, A. J. Beilstein J. Org. Chem. 2014, 10, 981-989.

[11] Lalevée, J.; Blanchard, N.; Tehfe, M.-A.; Peter, M.; Morlet-Savary, F.; Gigmes, D.; Fouassier, J.-P. Polymer Chem. 2011, 2, 1986-1991.

[12] Zubarev, V. E.; Belevskii, V. N.; Yarkov, S. P. Dokl. Akad. Nauk SSSR 1979, 244, 13921396. 
[13] a) Morlet-Savary, F. ; Klee, J. E. ; Pfefferkon, F. ; Fouassier, J. P. ; Lalevée, J. Macromol. Chem. Phys. 2015, 216, 2161-2170; b) Lalevée, J. ; Morlet-Savary, F.; Tehfe, M. A.; Graff, B.; Fouassier, J. P. Macromolecules 2012, 45, 5032-5039. 


\section{NMR Spectra}

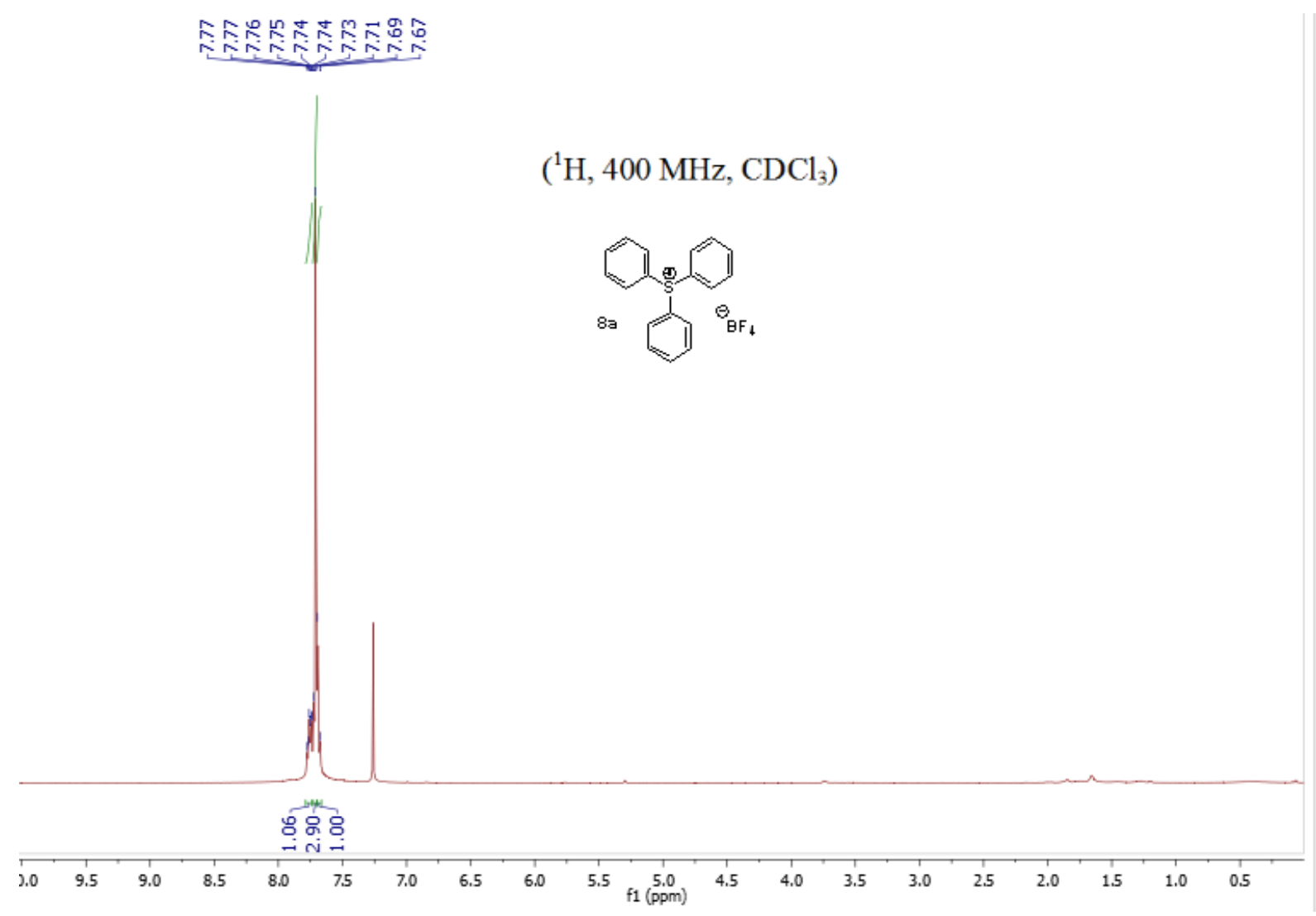

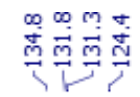

$\left({ }^{13} \mathrm{C}, 100 \mathrm{MHz}, \mathrm{CDCl}_{3}\right)$

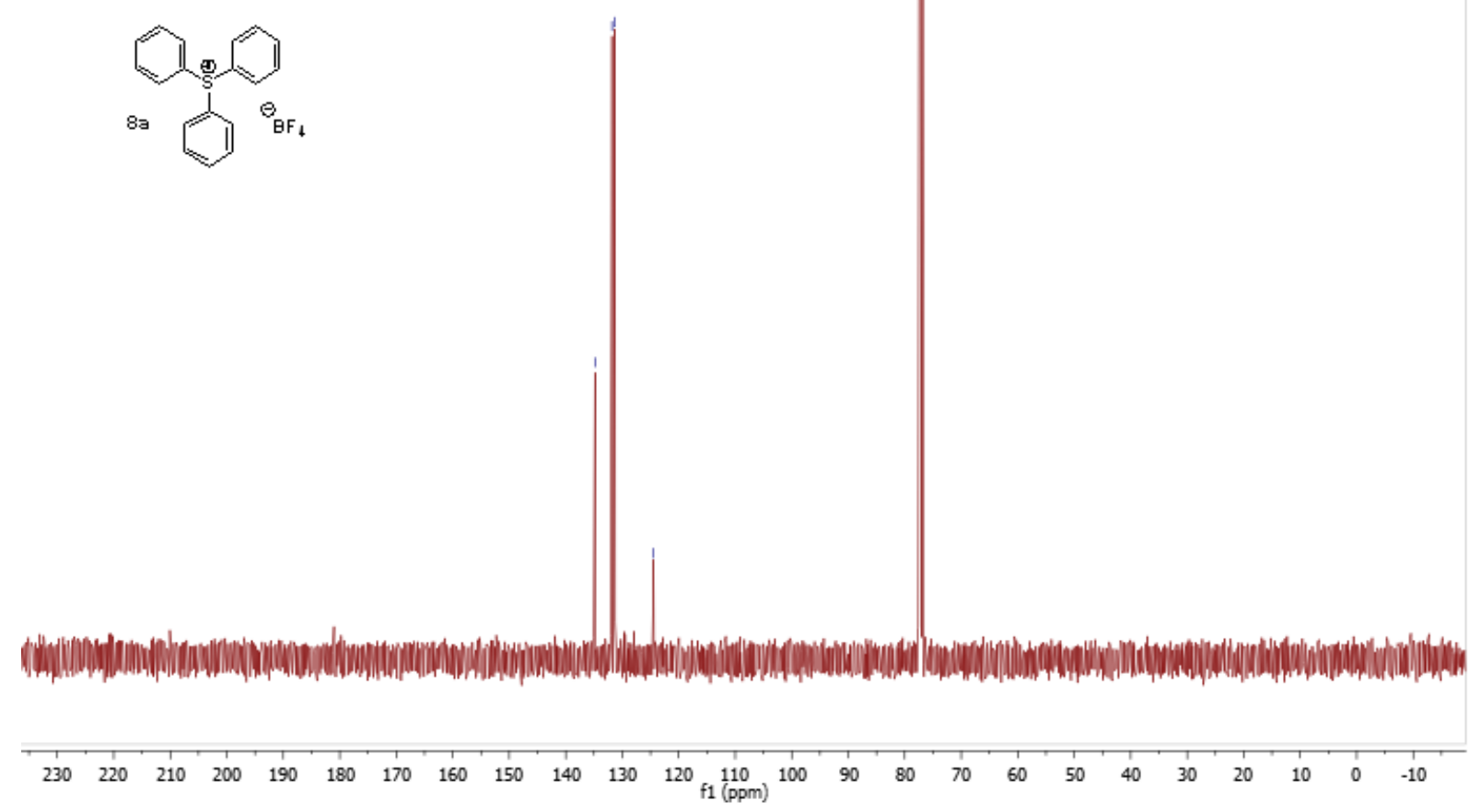


$\left({ }^{11} \mathrm{~B}, 128 \mathrm{MHz}, \mathrm{CDCl}_{3}\right)$

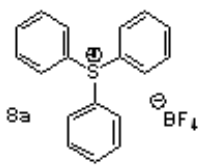

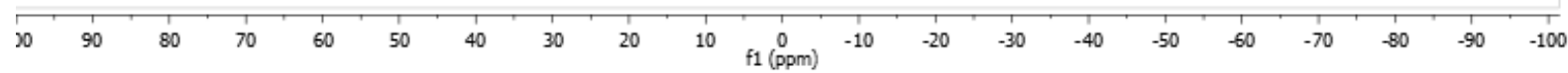

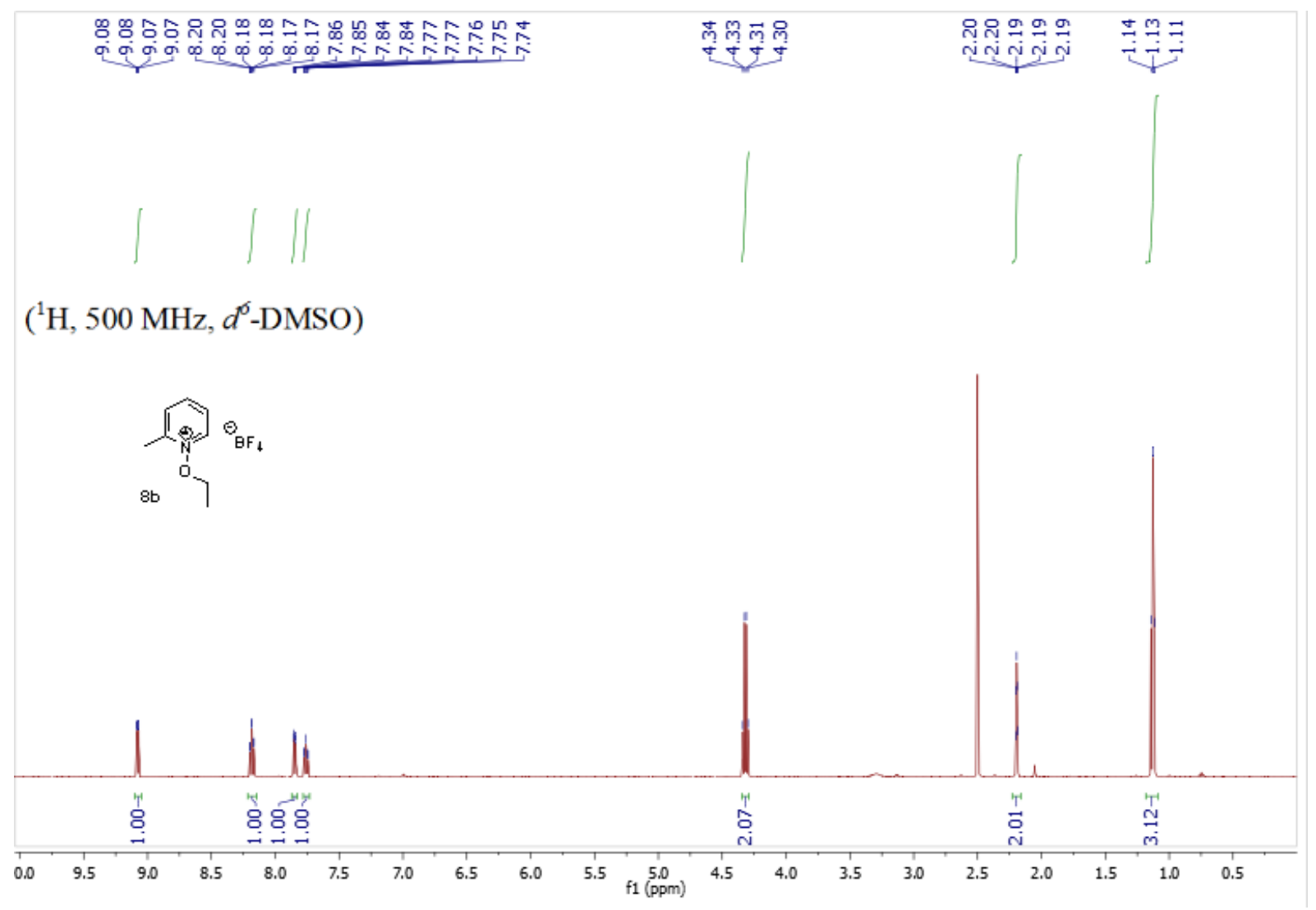


$\left({ }^{1} \mathrm{C}, 125 \mathrm{MHz}, d^{6}\right.$-DMSO)
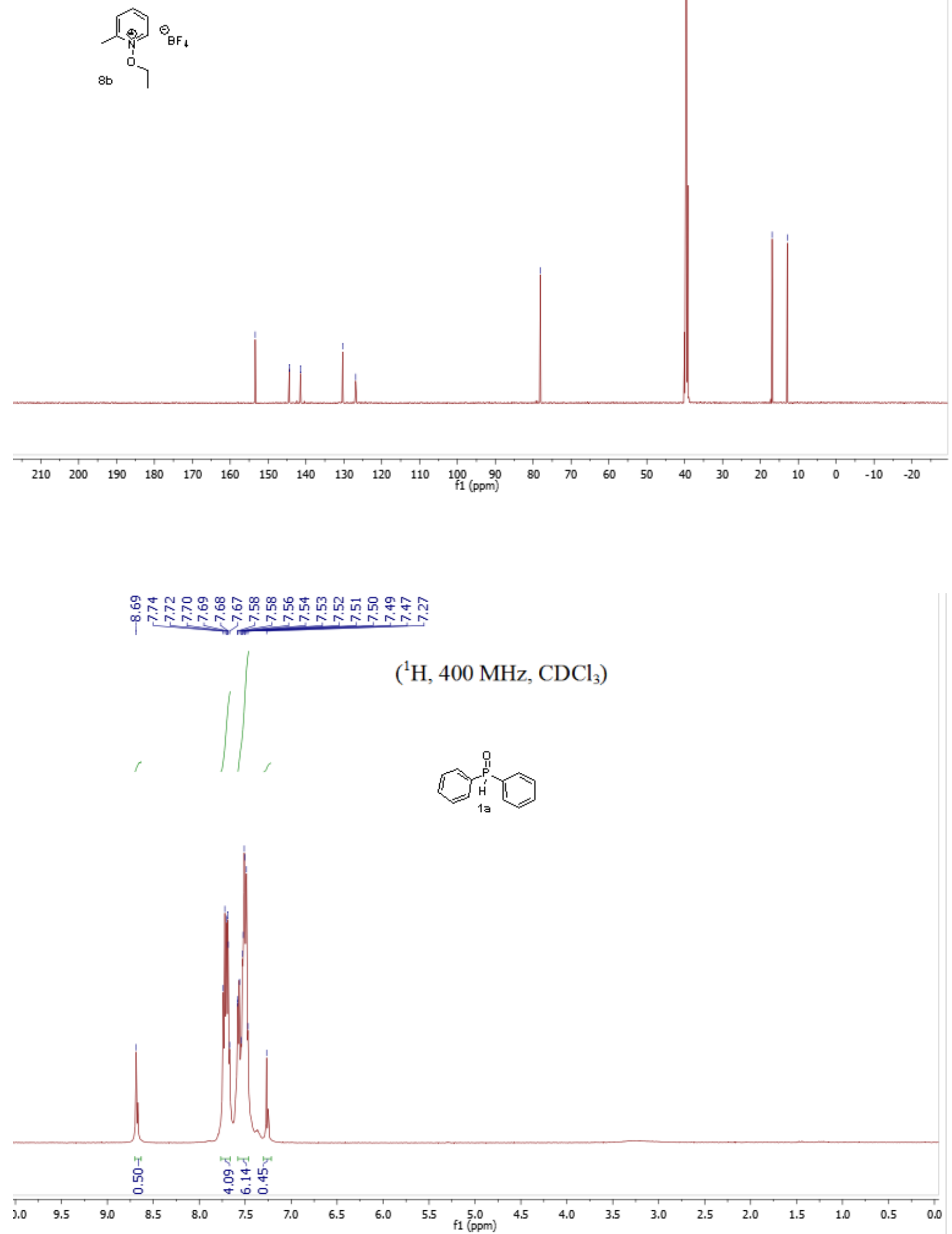


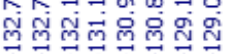

$\left({ }^{13} \mathrm{C}, 100 \mathrm{MHz}, \mathrm{CDCl}_{3}\right)$<smiles></smiles>

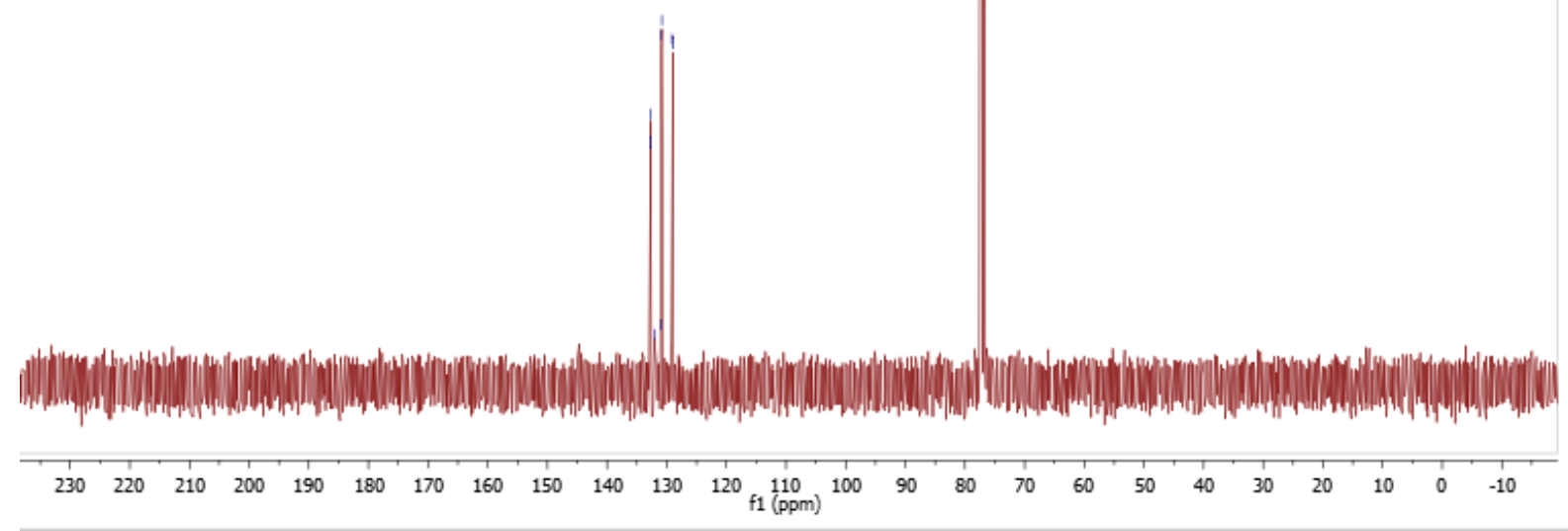

ฟู่

$\left({ }^{31} \mathrm{P}, 162 \mathrm{MHz}, \mathrm{CDCl}_{3}\right)$

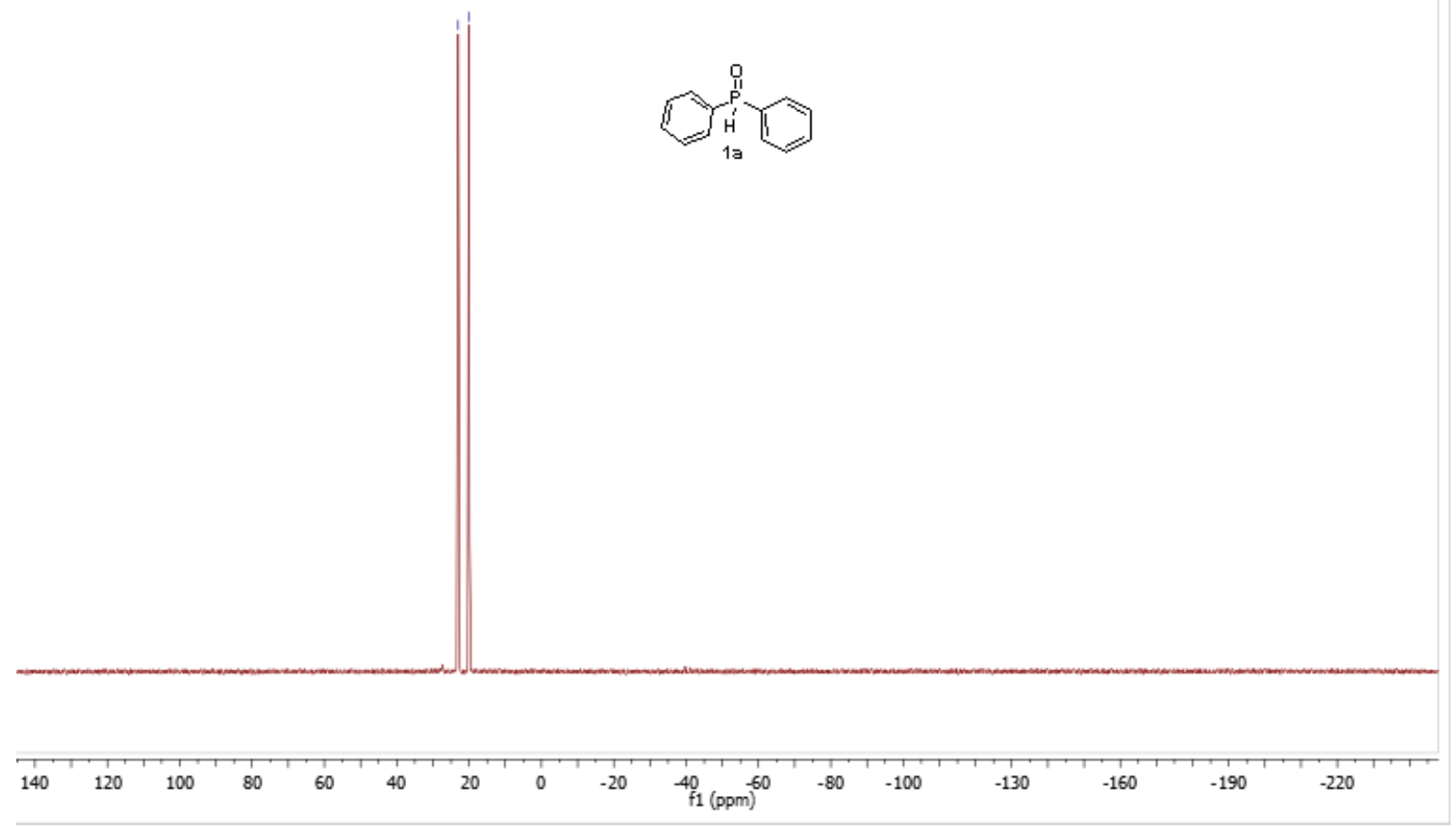



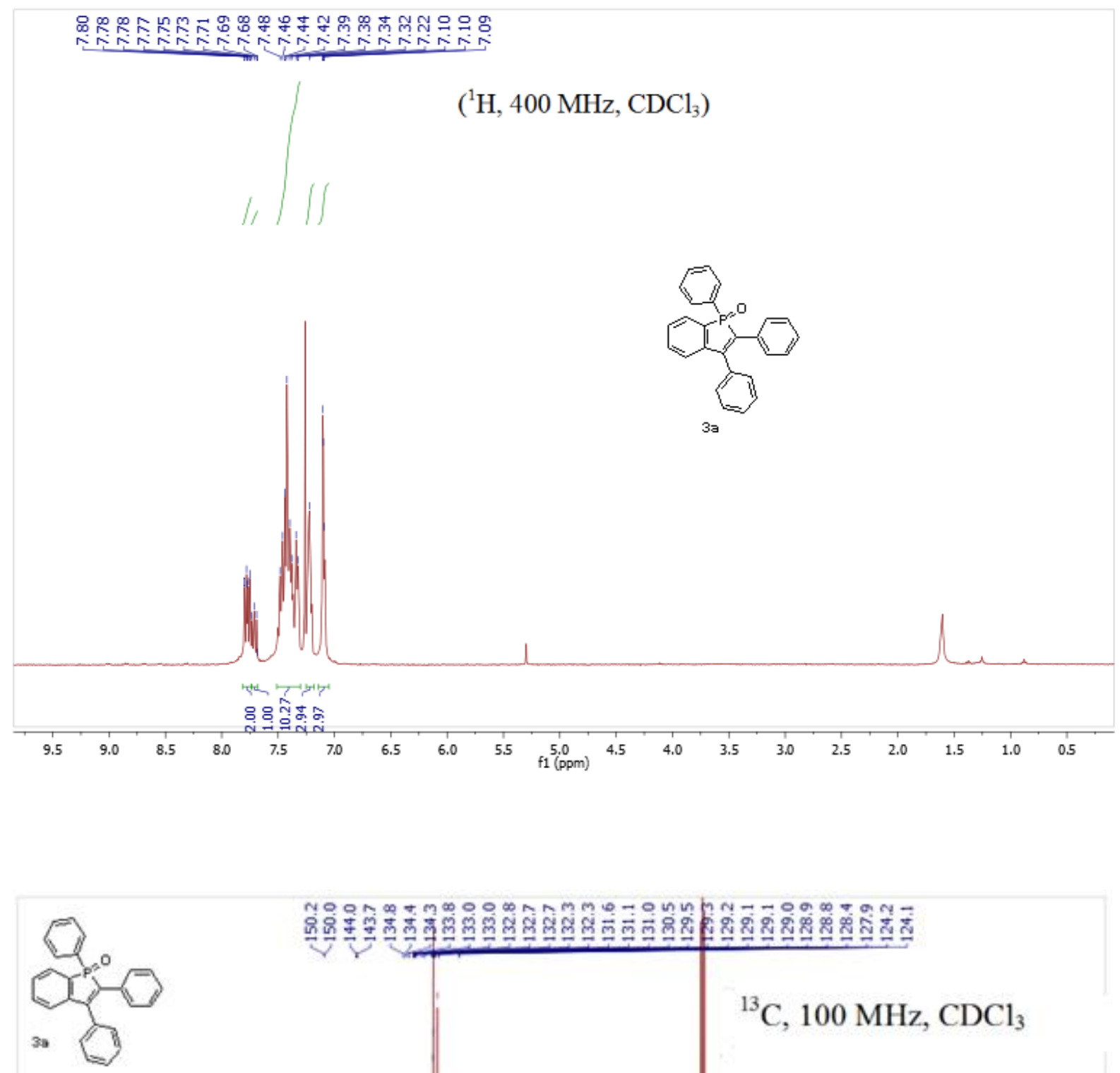

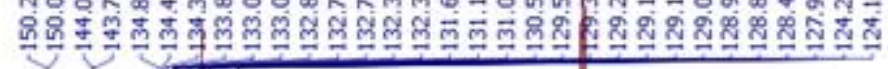

${ }^{13} \mathrm{C}, 100 \mathrm{MHz}, \mathrm{CDCl}_{3}$
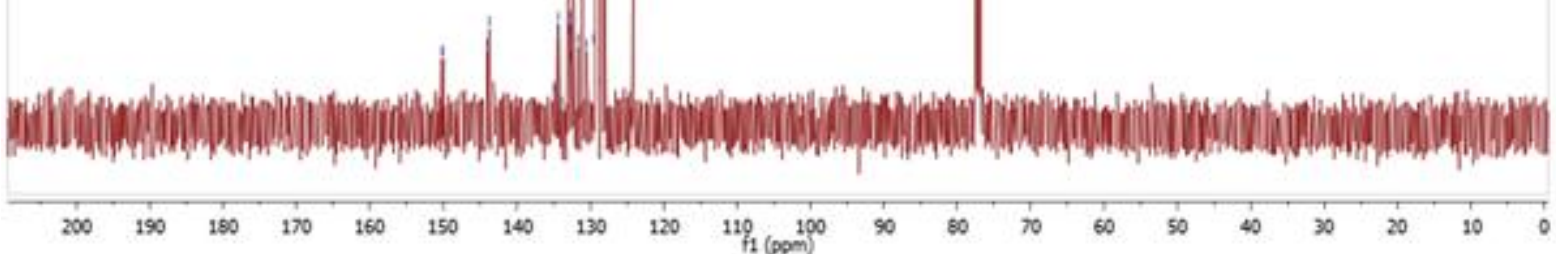


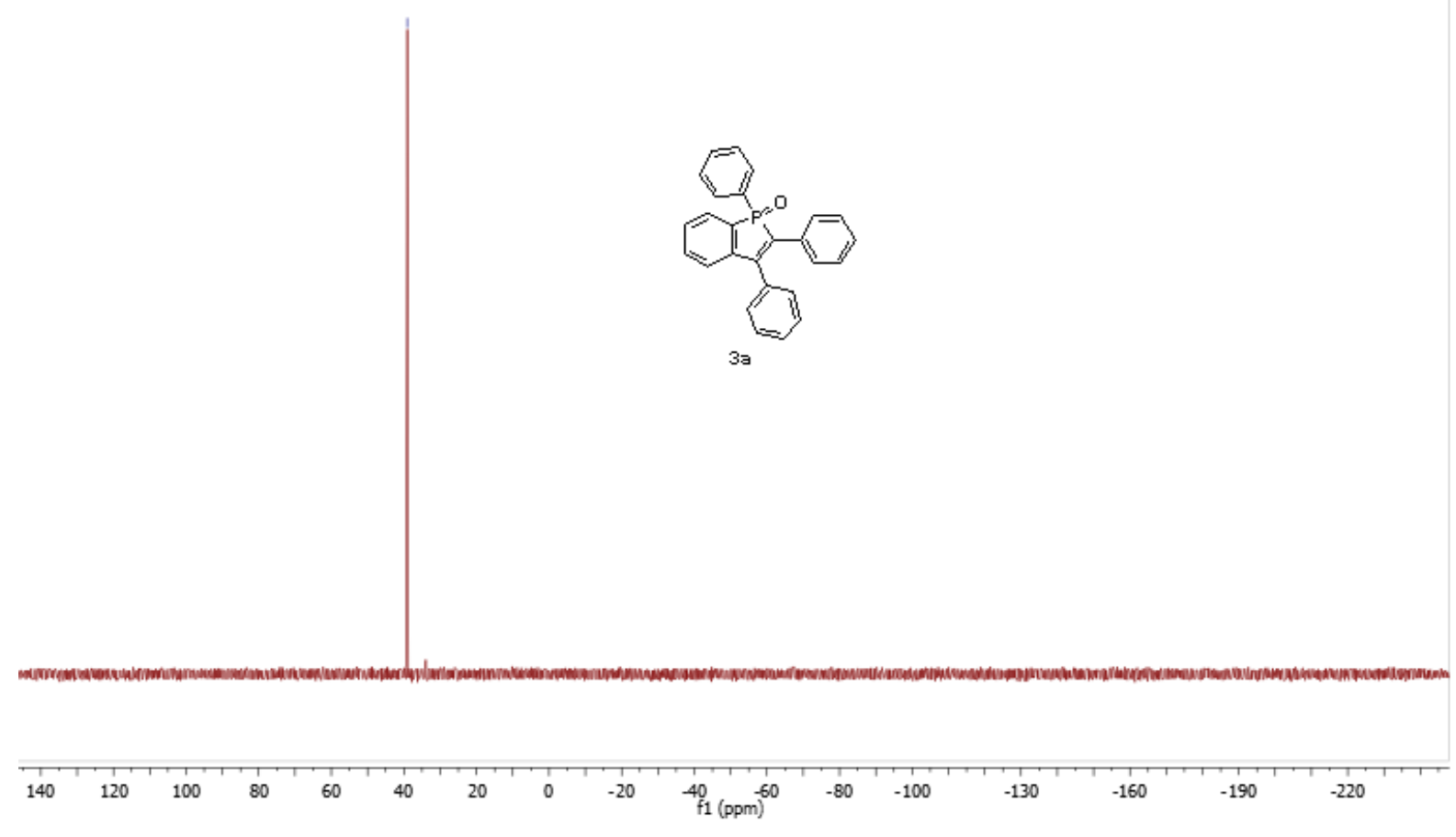

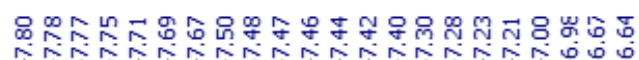

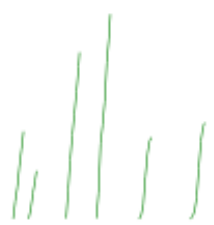

$\left({ }^{1} \mathrm{H}, 400 \mathrm{MHz}, \mathrm{CDCl}_{3}\right)$

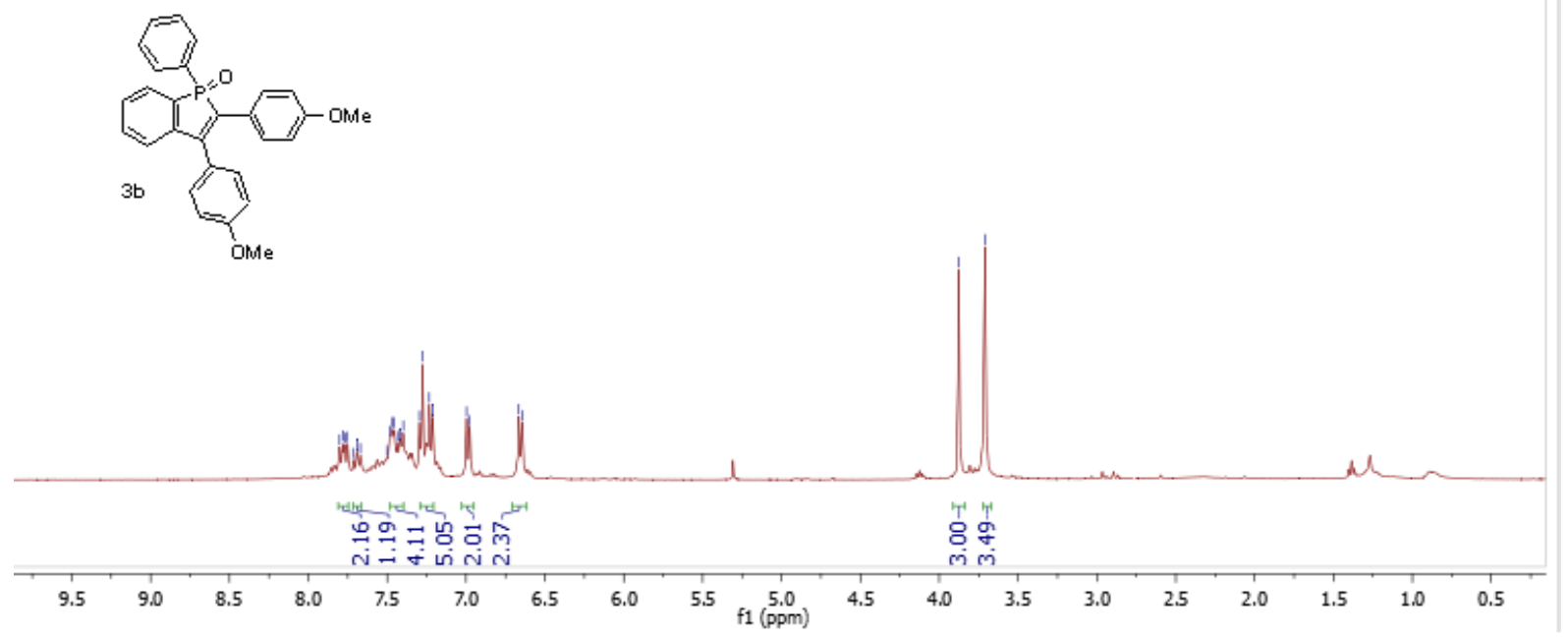



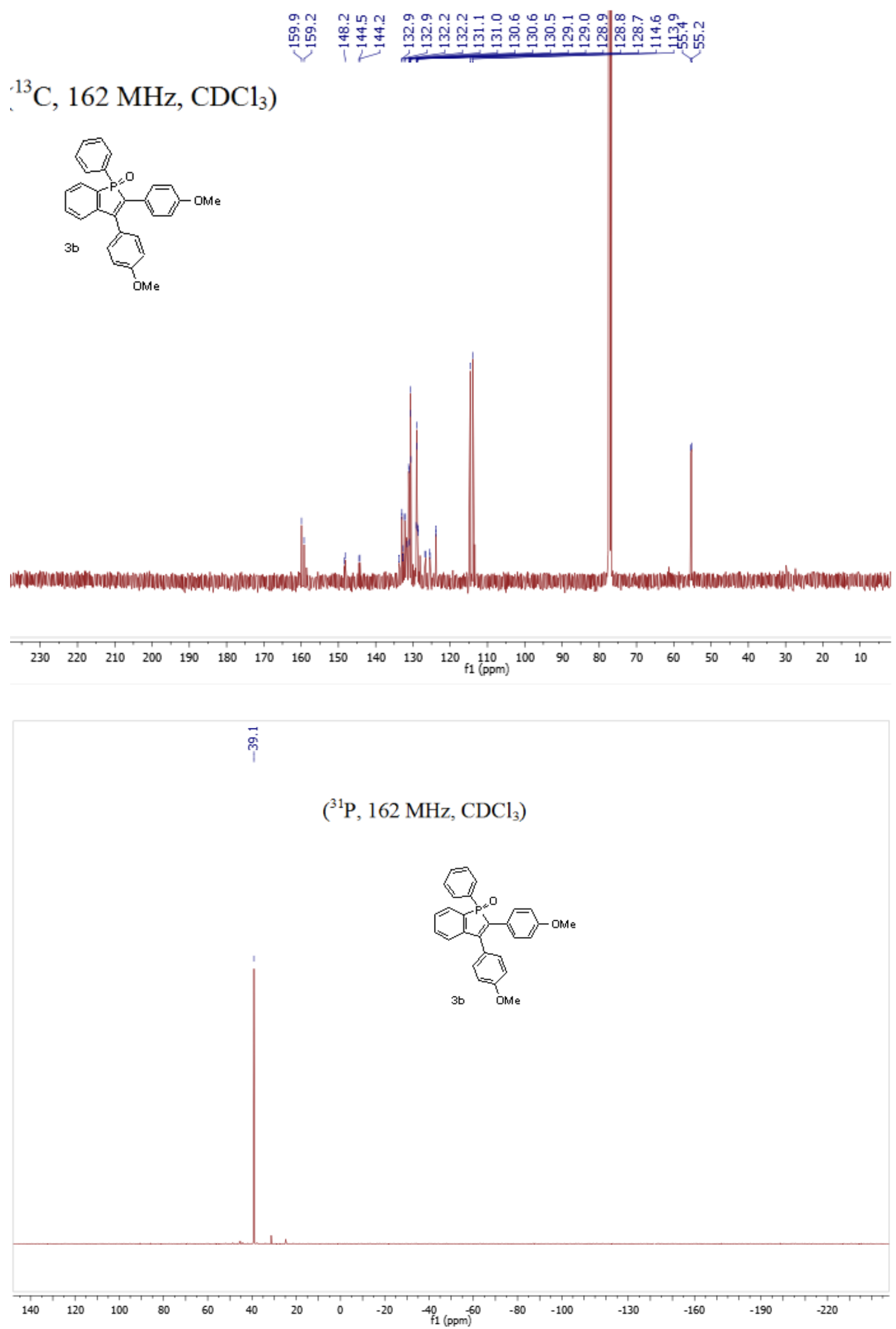

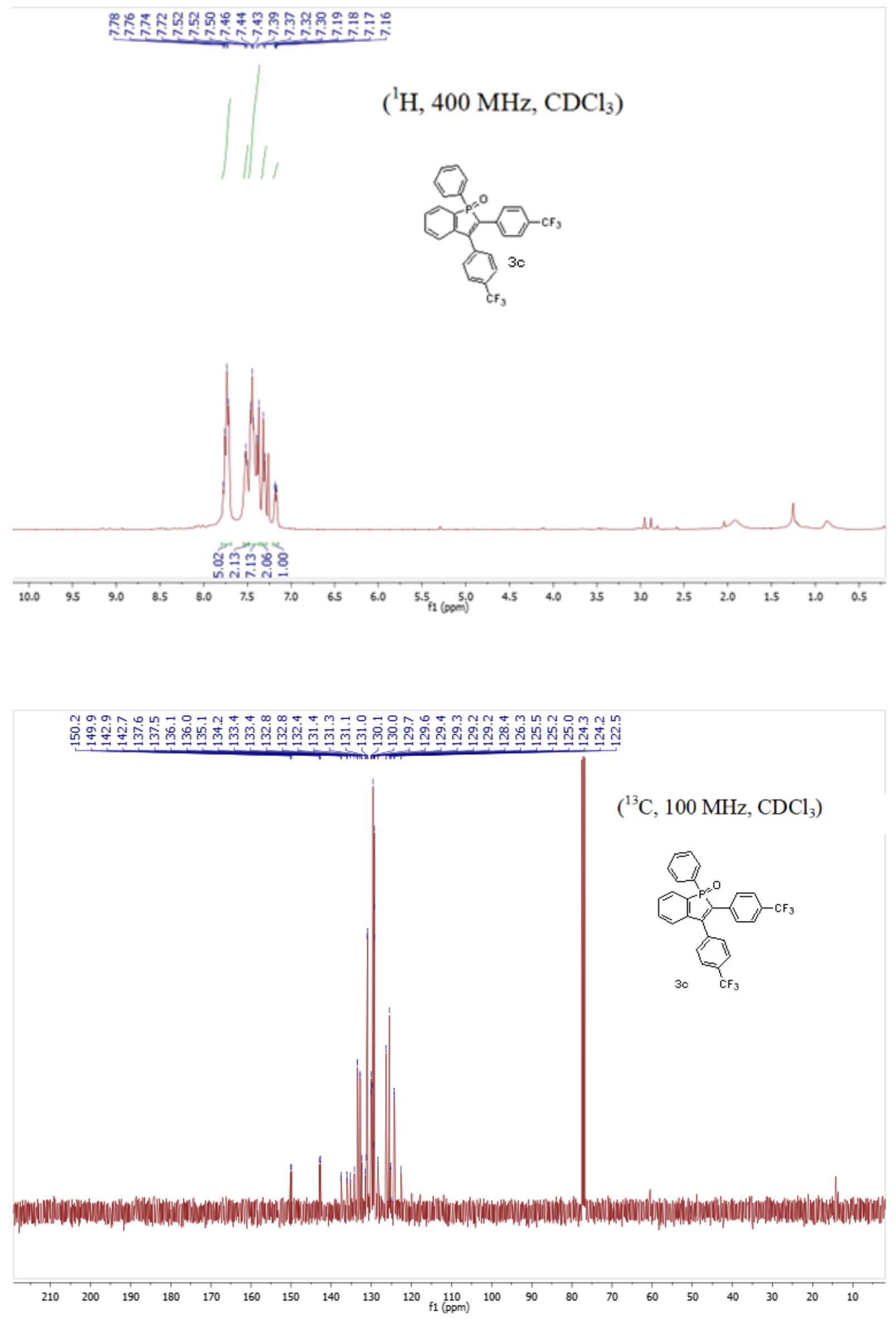
Quint, Morlet-Savary, Lohier, Lalevée, Gaumont, and Lakhdar
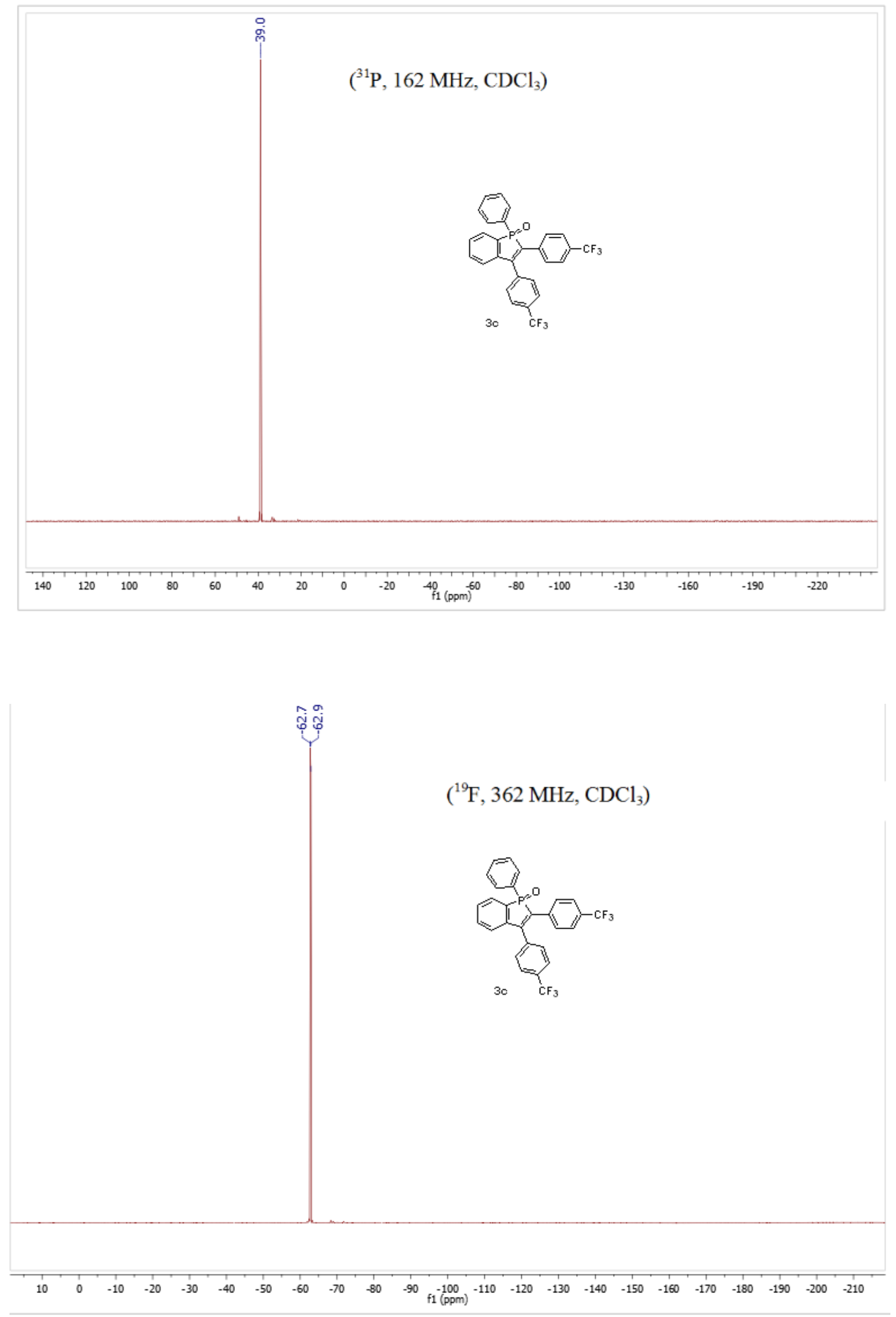

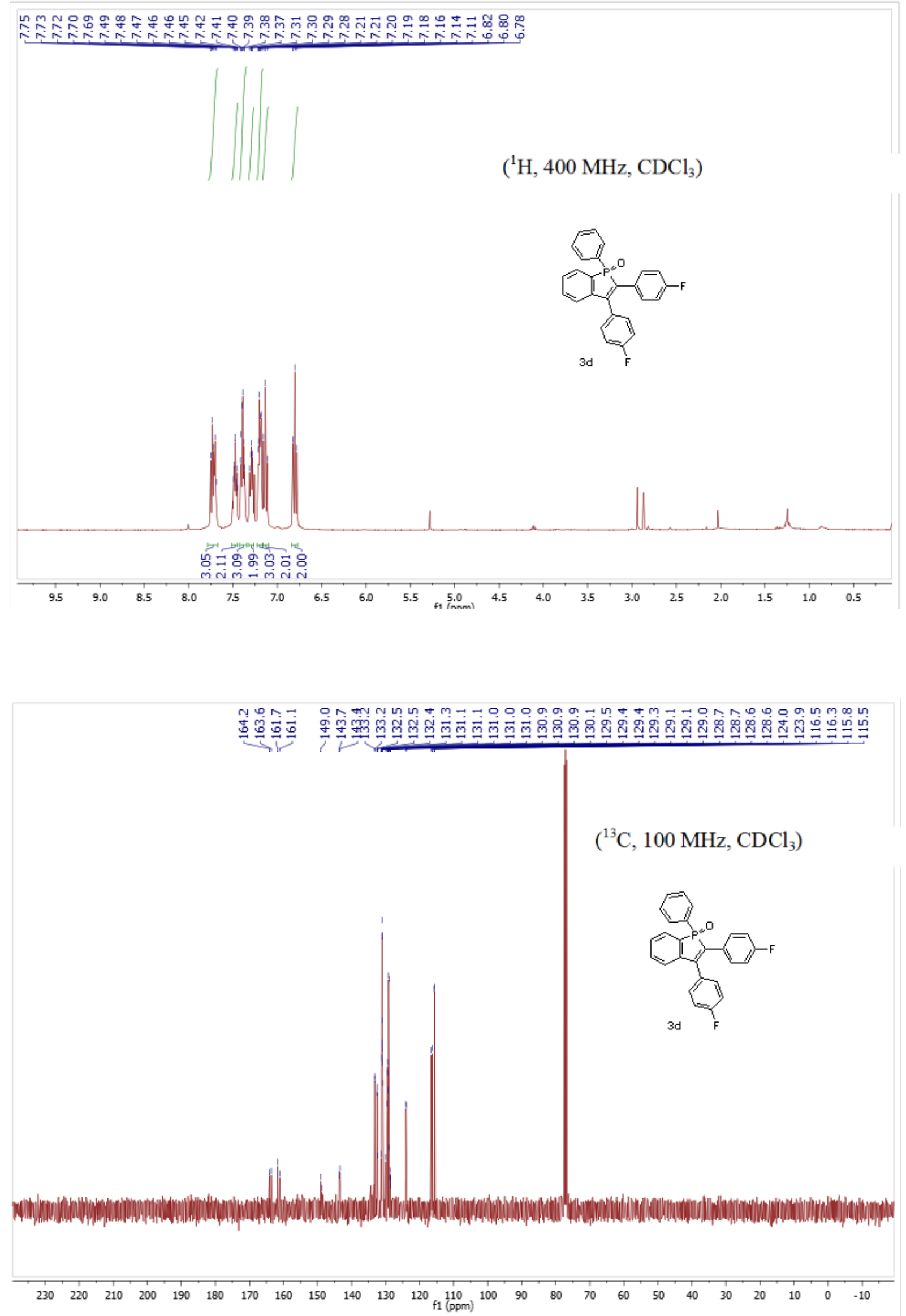
$\left({ }^{31} \mathrm{P}, 162 \mathrm{MHz}, \mathrm{CDCl}_{3}\right)$
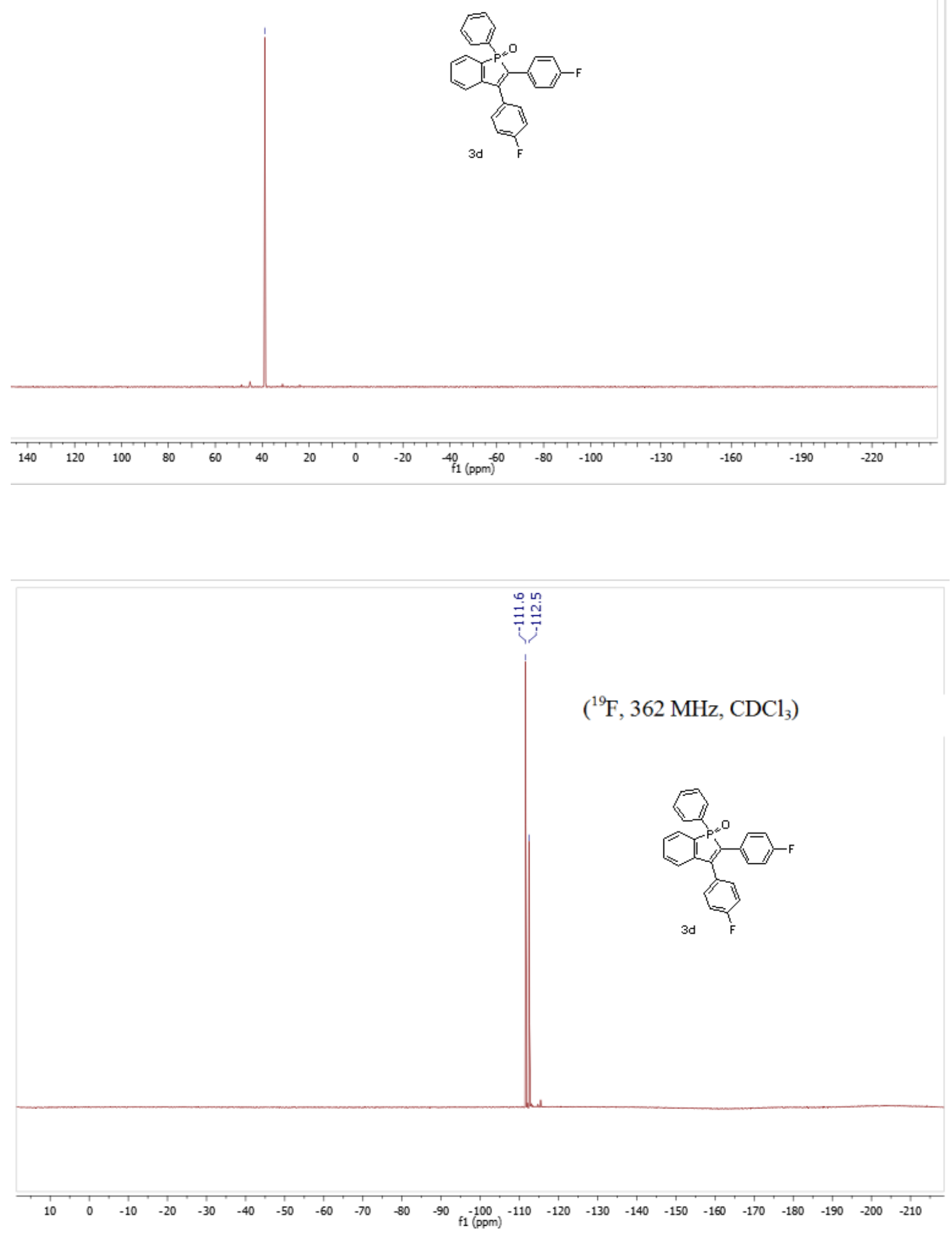


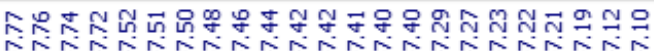

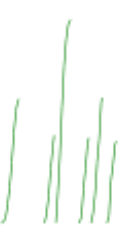

$\left({ }^{1} \mathrm{H}, 400 \mathrm{MHz}, \mathrm{CDCl}_{3}\right)$

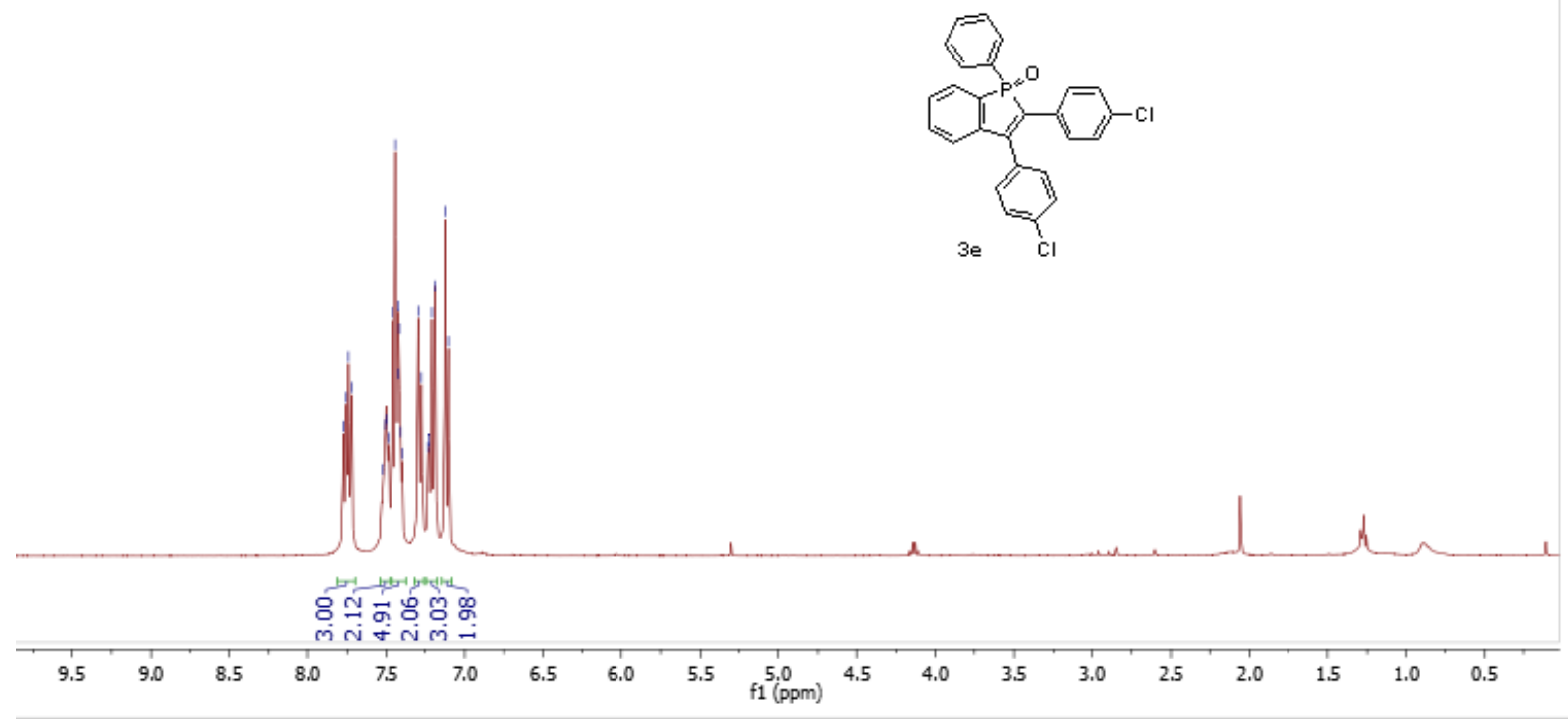

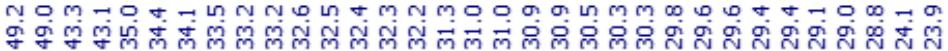

$\left({ }^{13} \mathrm{C}, 100 \mathrm{MHz}, \mathrm{CDCl}_{3}\right)$

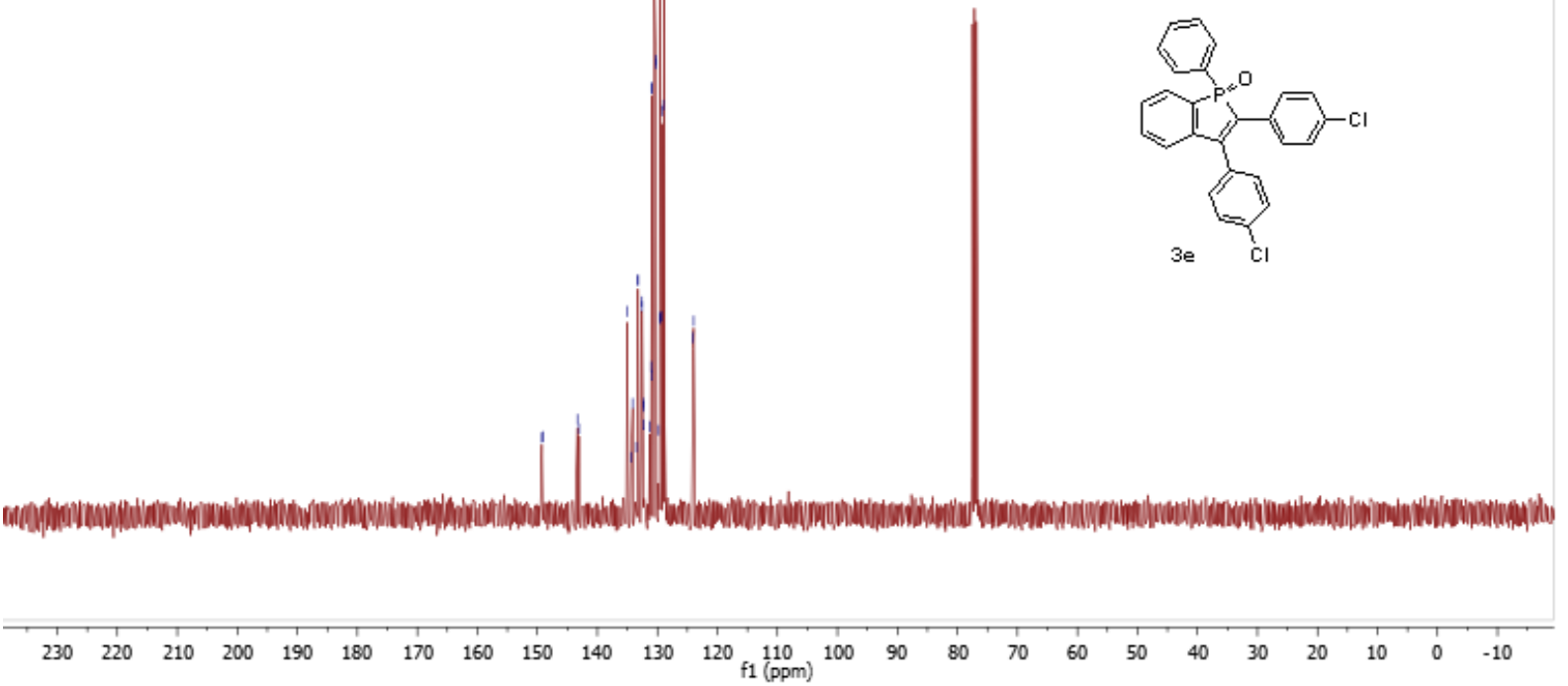




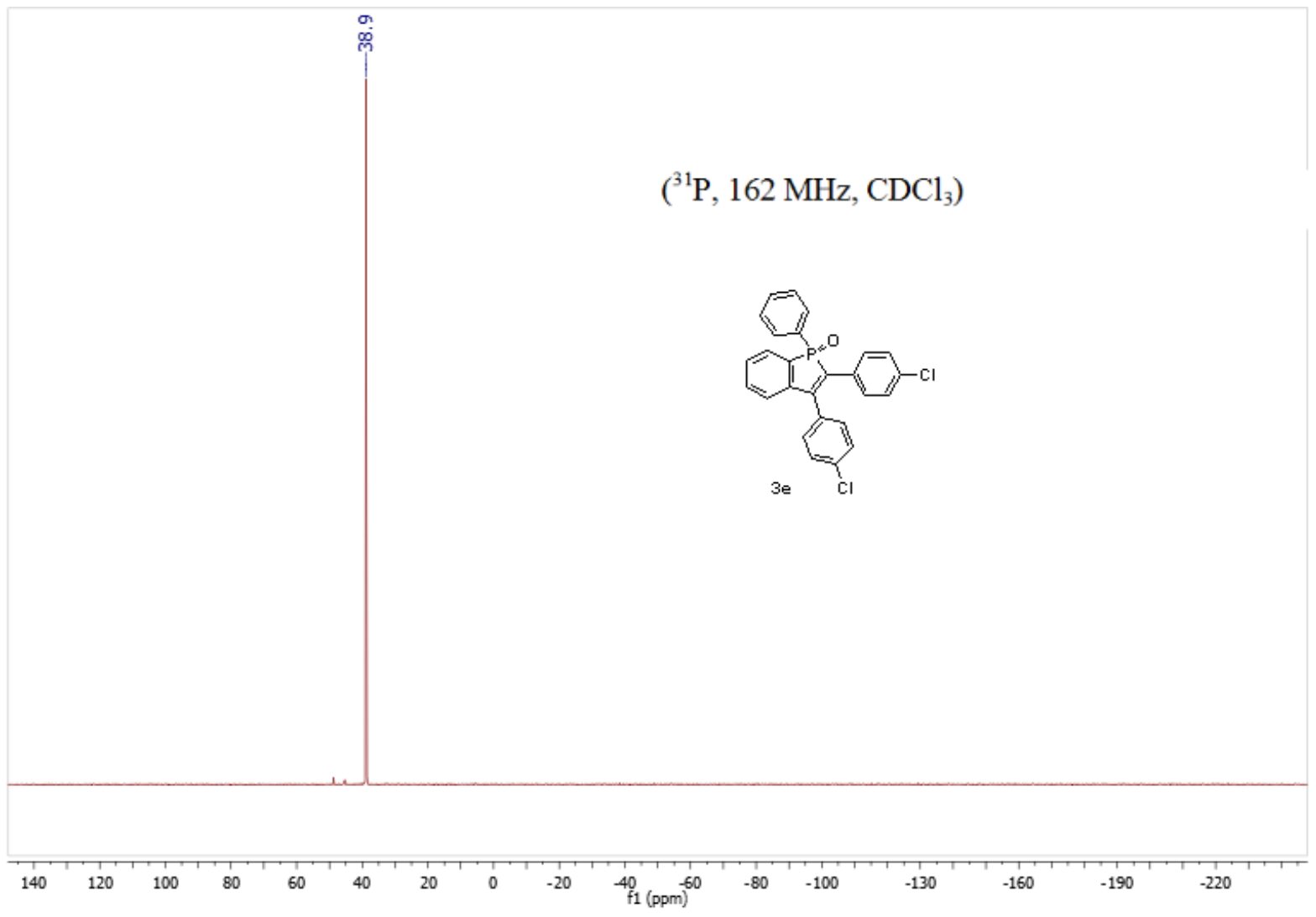

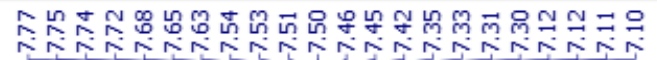

$$
\stackrel{\vec{\sigma}}{i} \underset{\substack{\infty \\ i}}{\infty}
$$

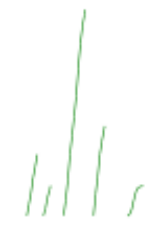

$\left({ }^{1} \mathrm{H}, 400 \mathrm{MHz}, \mathrm{CDCl}_{3}\right)$

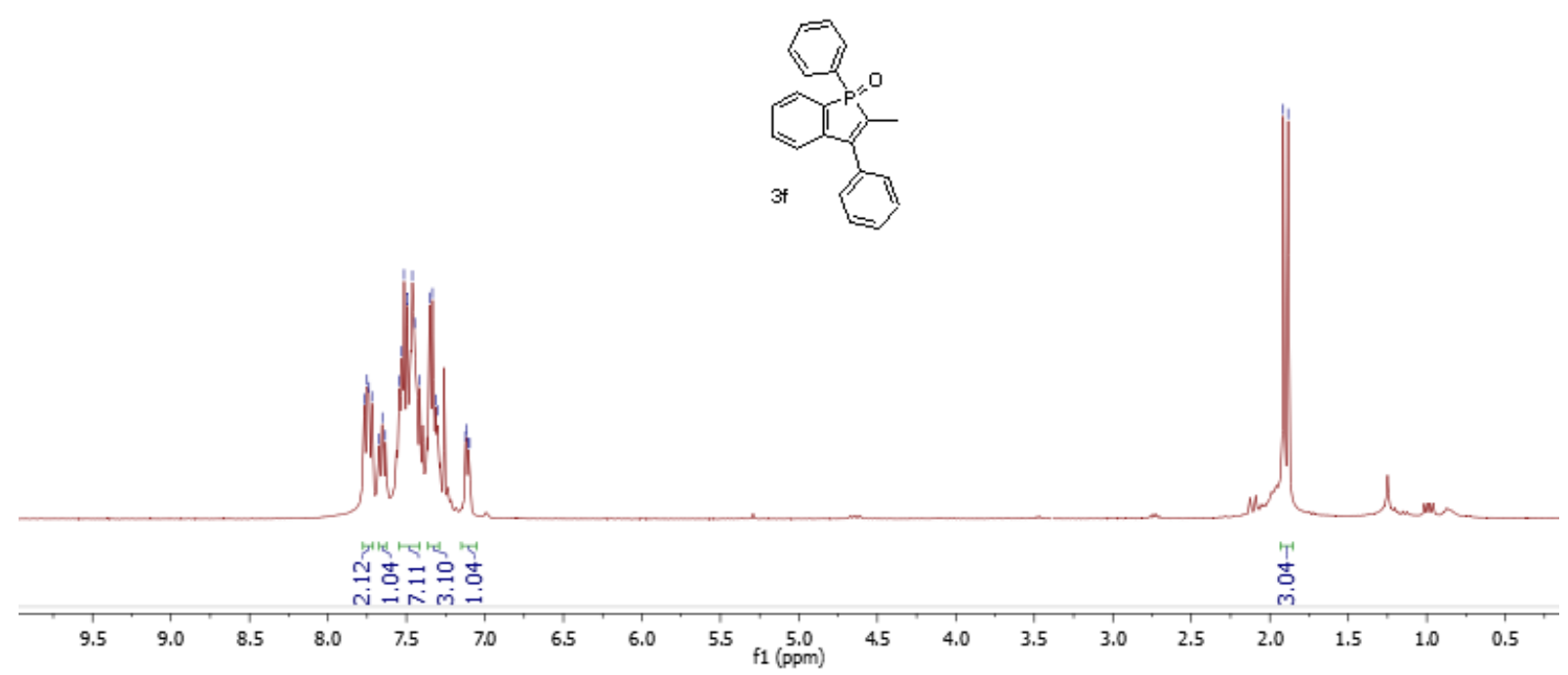




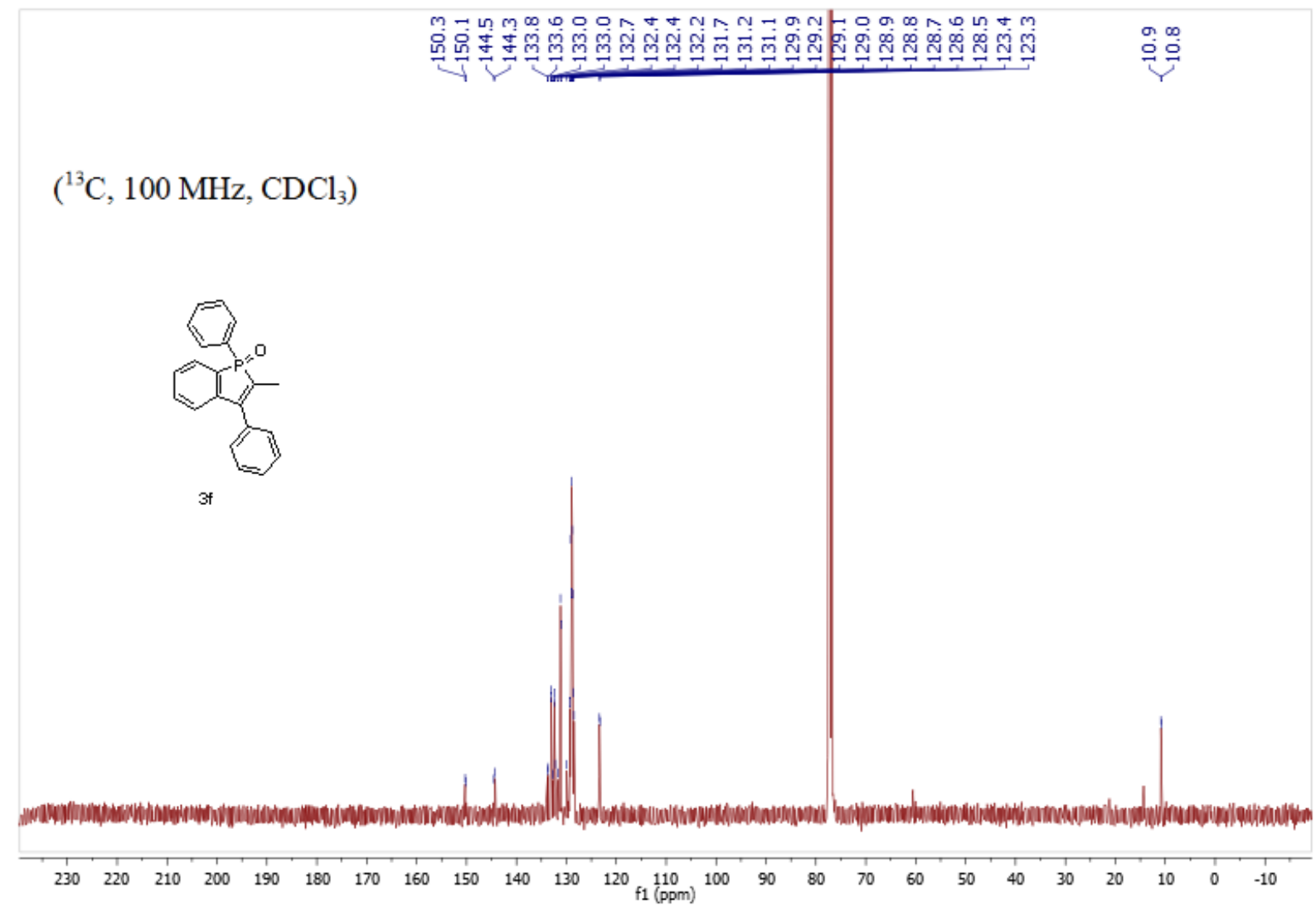

$\left({ }^{31} \mathrm{P}, 162 \mathrm{MHz}, \mathrm{CDCl}_{3}\right)$
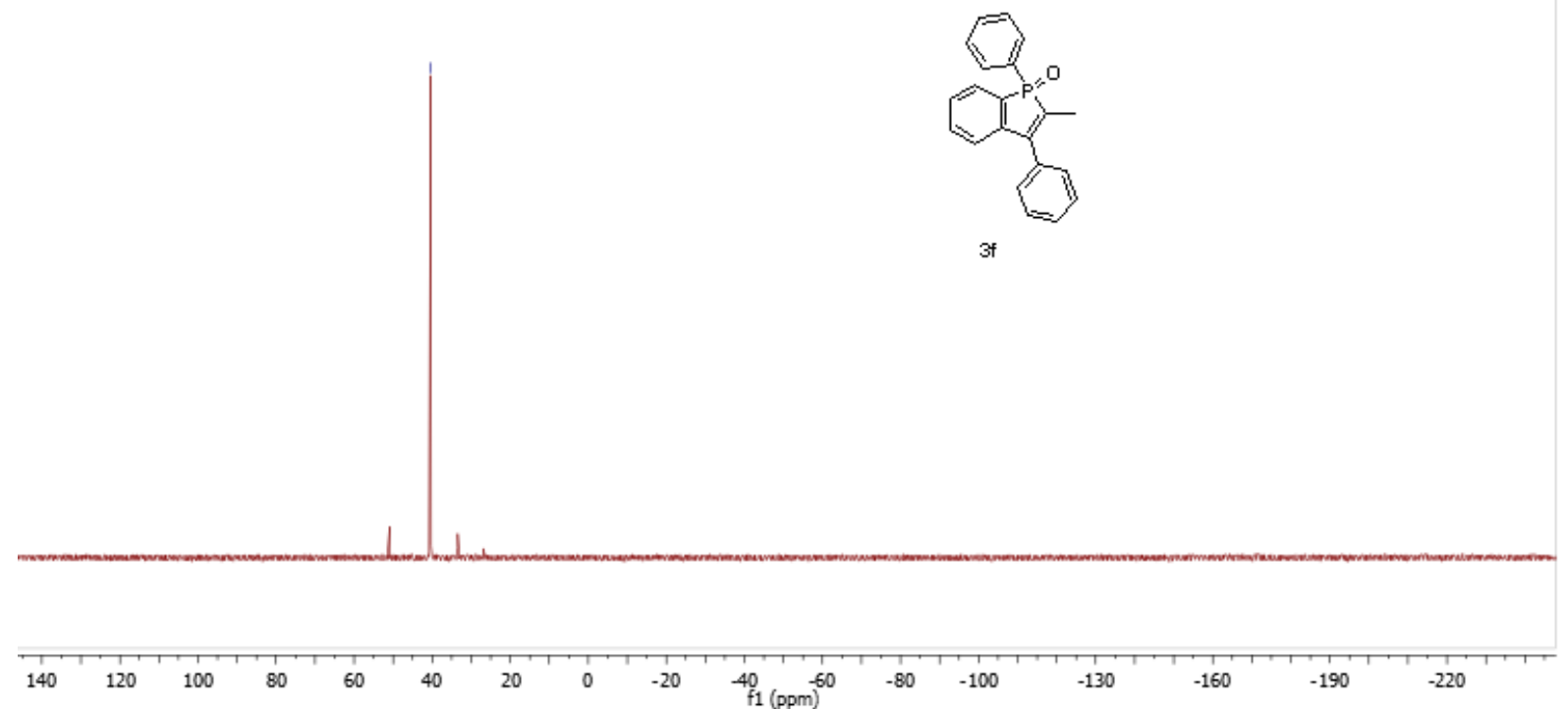


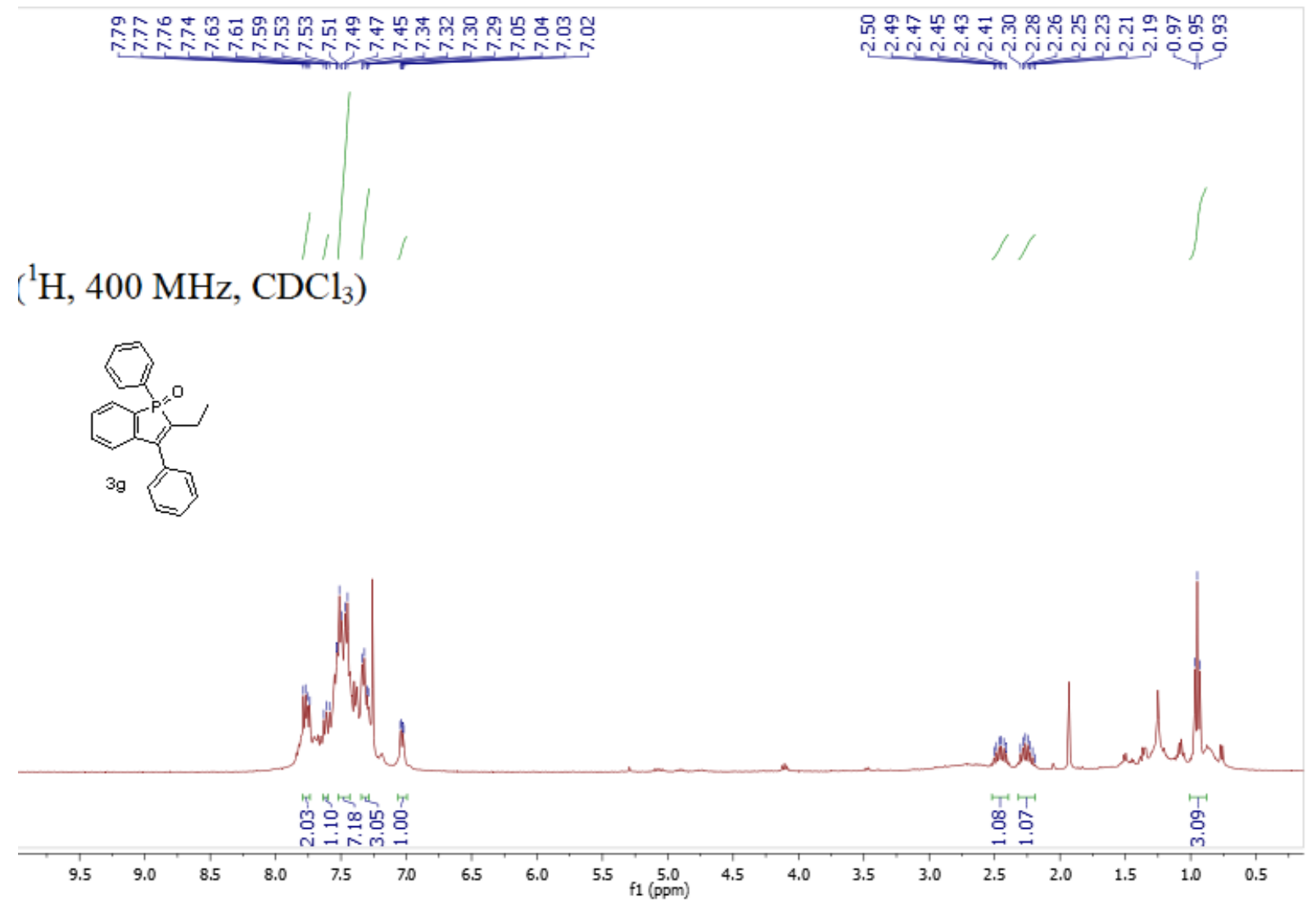

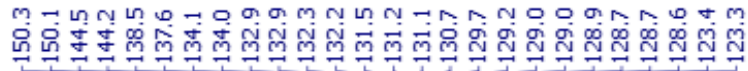

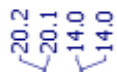

$\left({ }^{13} \mathrm{C}, 100 \mathrm{MHz}, \mathrm{CDCl}_{3}\right)$
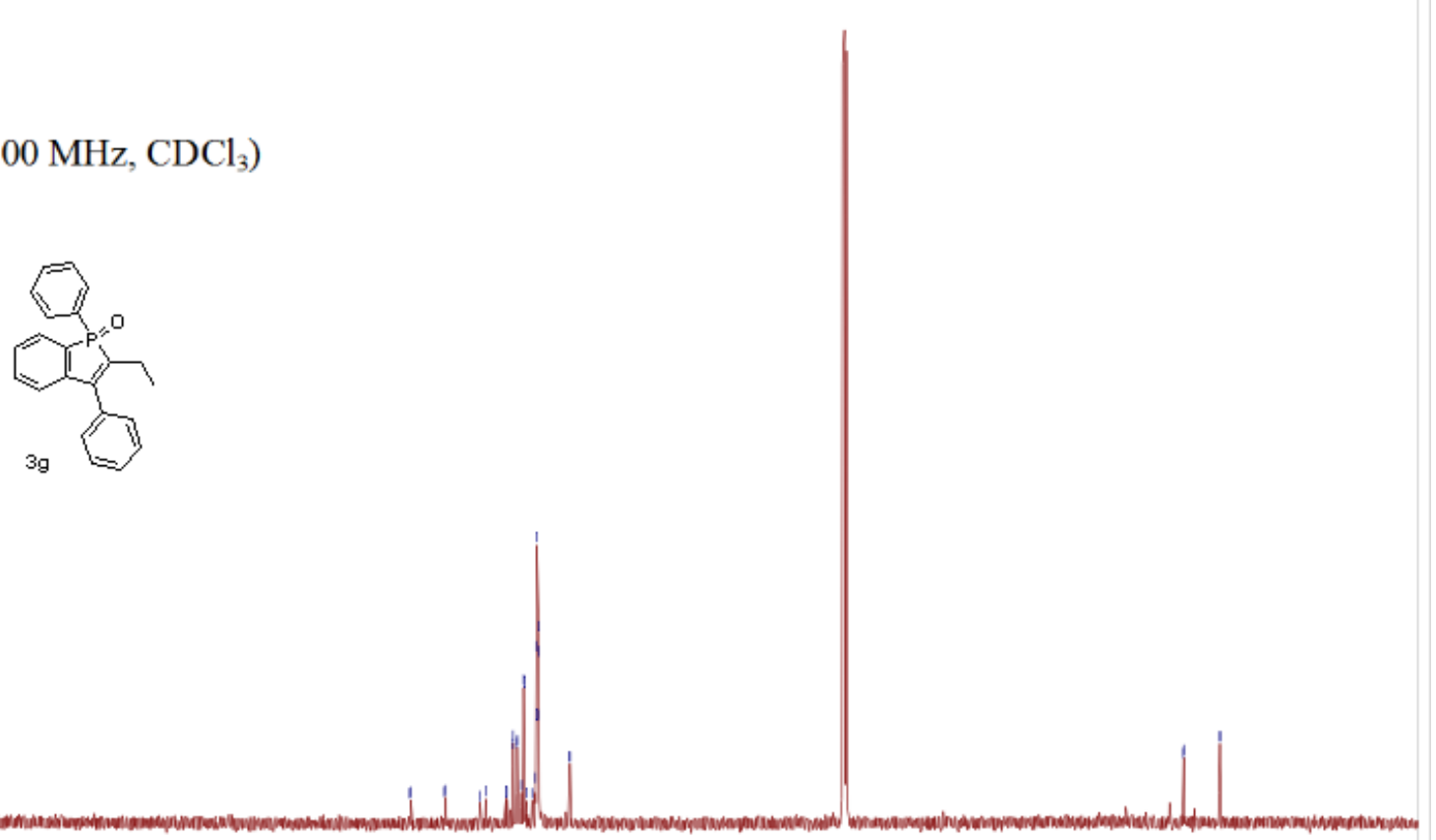

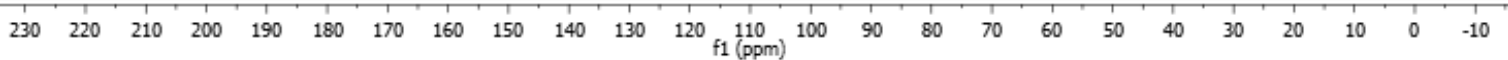


$\left({ }^{31} \mathrm{P}, 162 \mathrm{MHz}, \mathrm{CDCl}_{3}\right)$

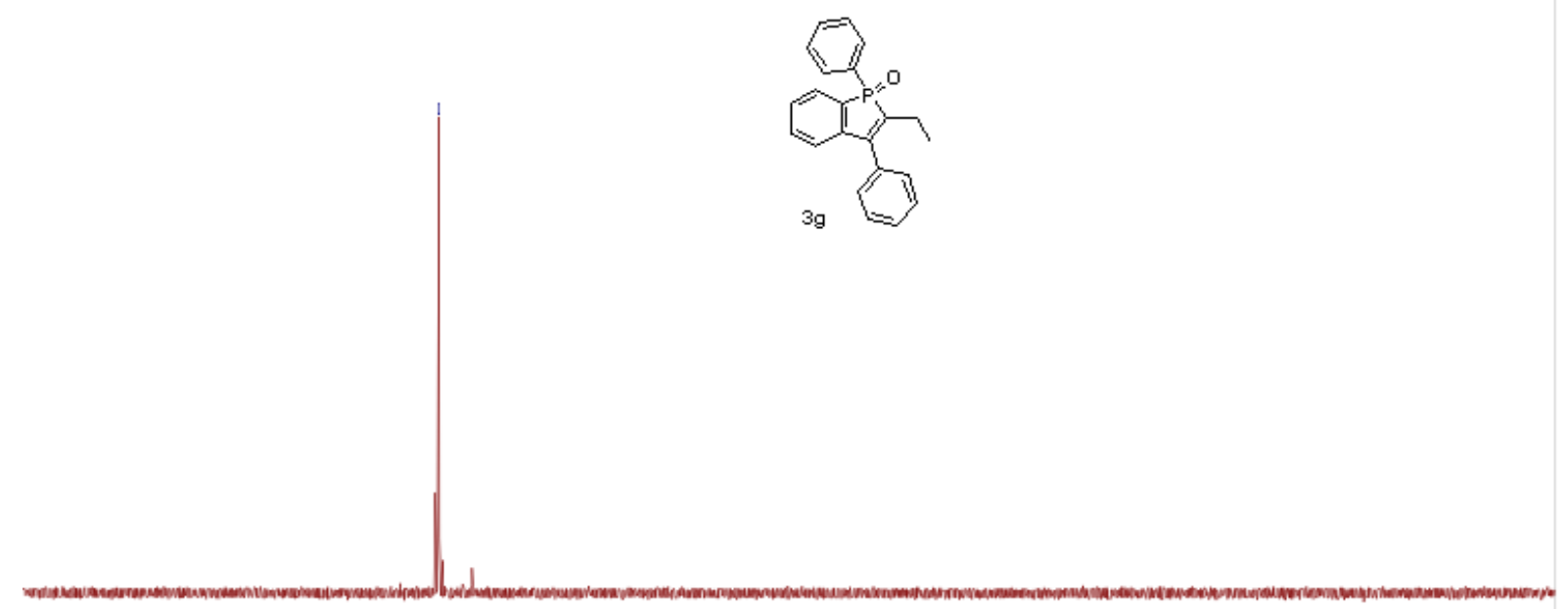

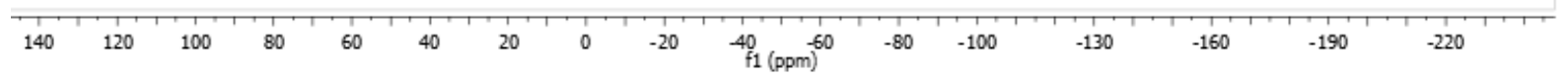

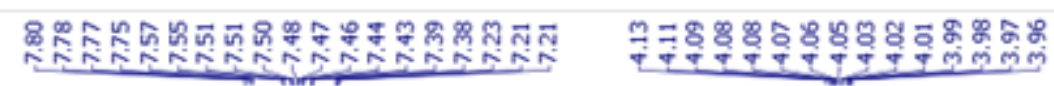

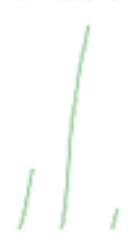

$\left({ }^{1} \mathrm{H}, 400 \mathrm{MHz}, \mathrm{CDCl}_{3}\right)$<smiles></smiles>
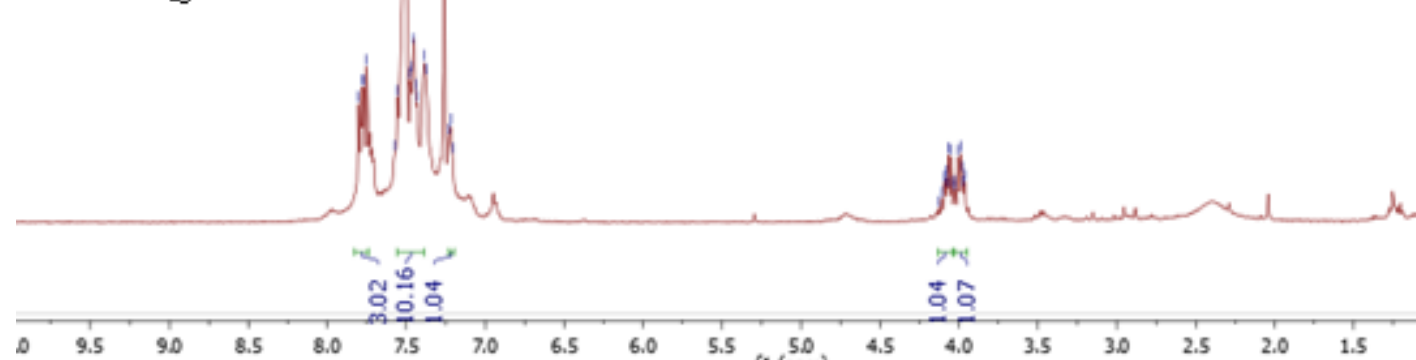

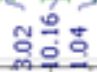

int $\underline{\underline{a}} \underline{\underline{0}}$

'丁'

$\begin{array}{lllllllllll}10 & 9.5 & 9.0 & 8.5 & 8.0 & 7.5 & 7.0 & 6.5 & 6.0 & 5.5 & \left.\begin{array}{lll}5.0 \\ \mathrm{f} 1(\mathrm{pom})\end{array}\right)\end{array}$ 


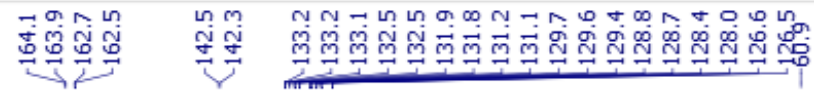

$\left({ }^{13} \mathrm{C}, 100 \mathrm{MHz}, \mathrm{CDCl}_{3}\right)$
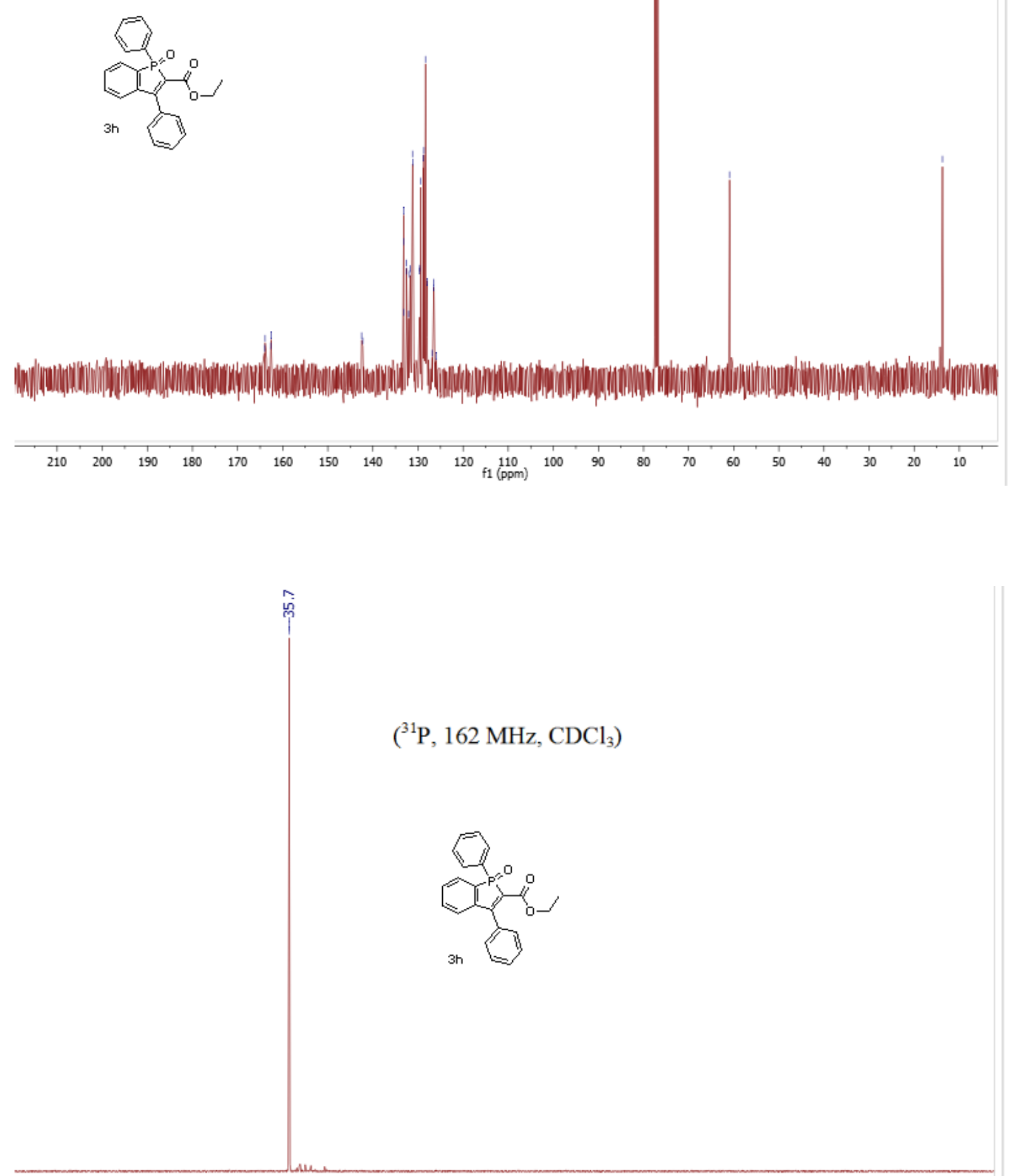

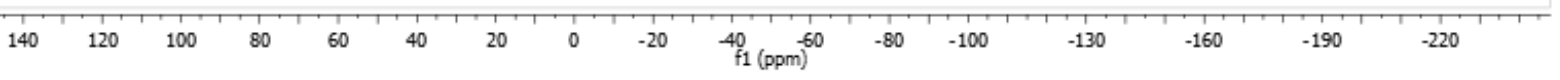




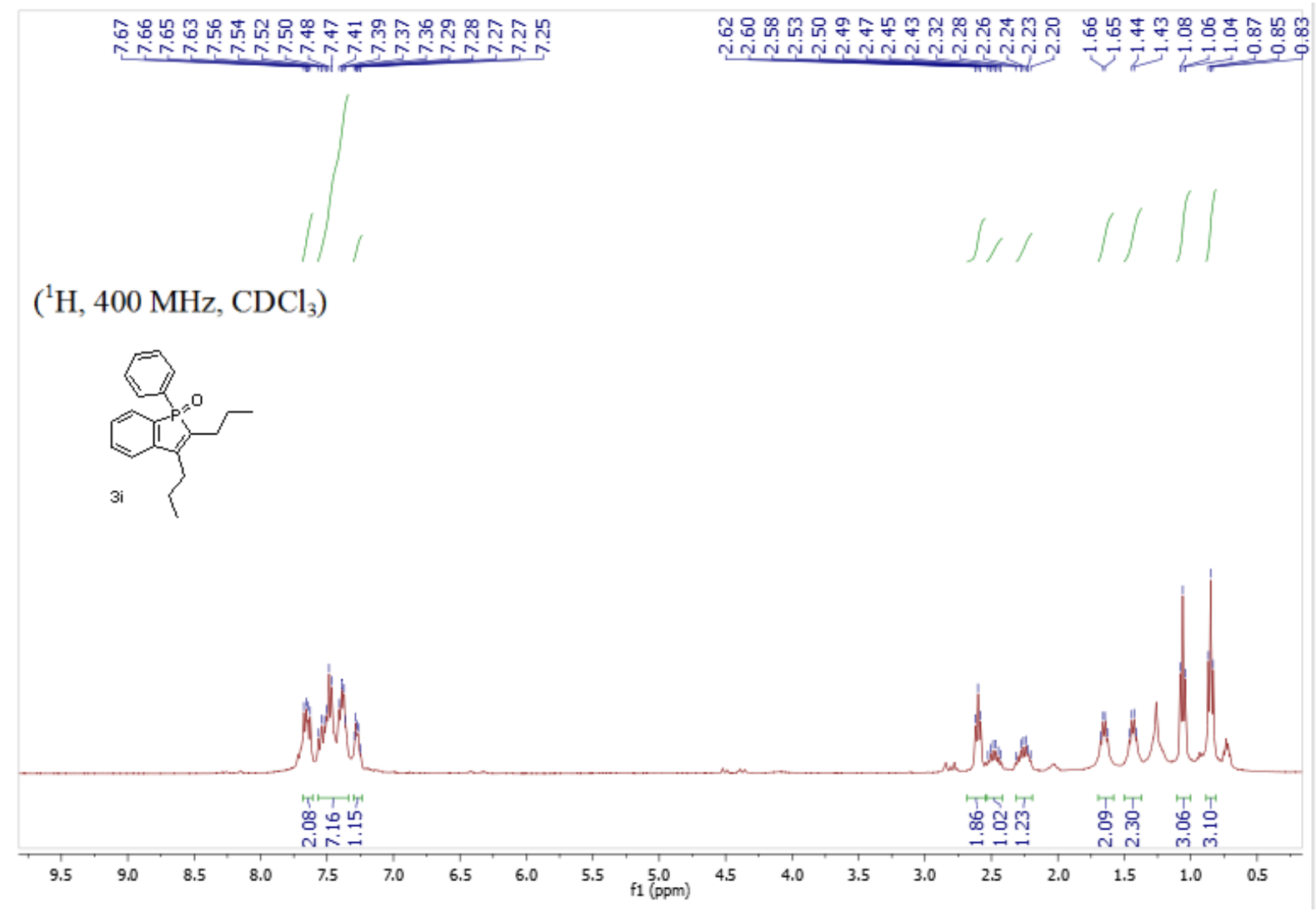

$\left({ }^{13} \mathrm{C}, 100 \mathrm{MHz}, \mathrm{CDCl}_{3}\right)$
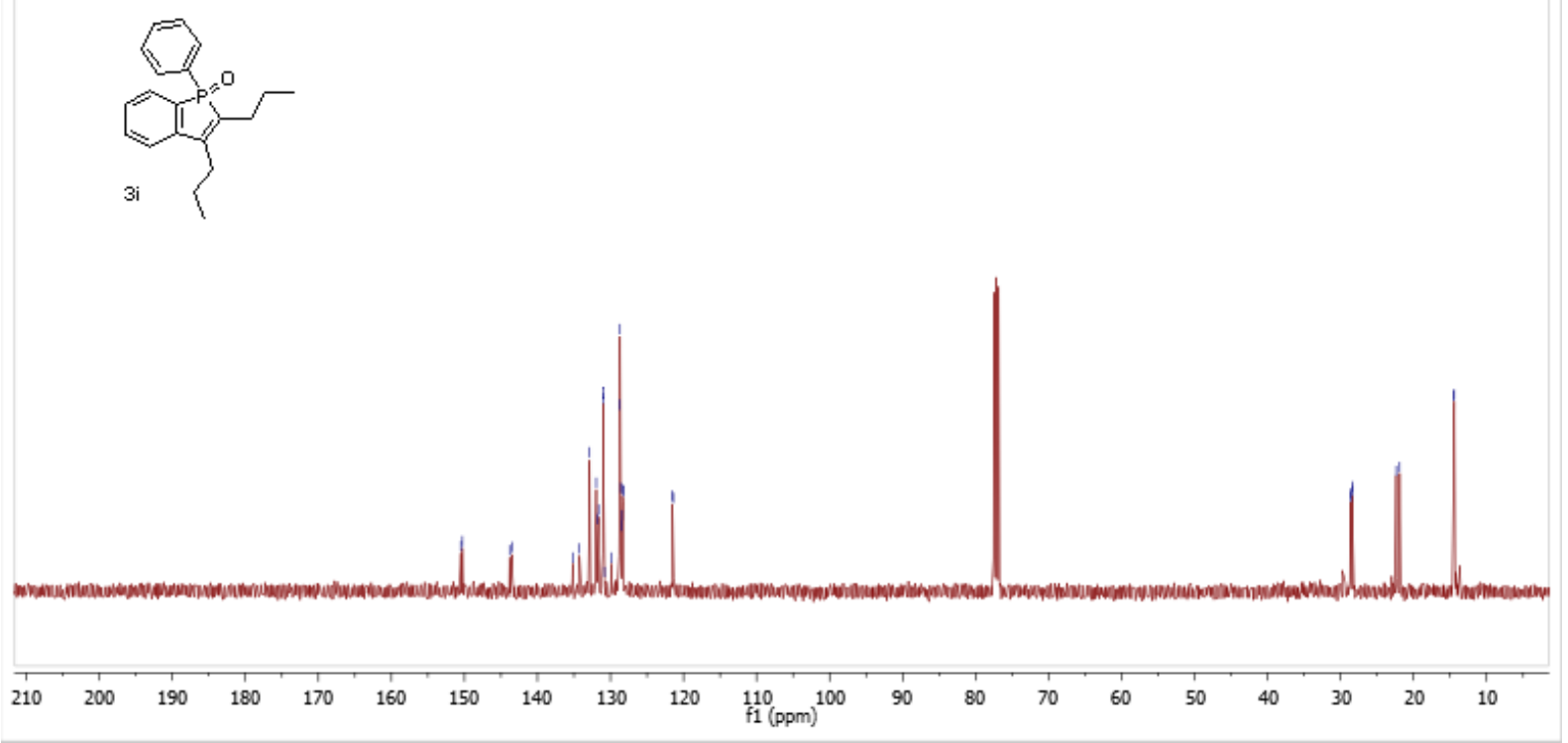
$\left({ }^{31} \mathrm{P}, 162 \mathrm{MHz}, \mathrm{CDCl}_{3}\right)$
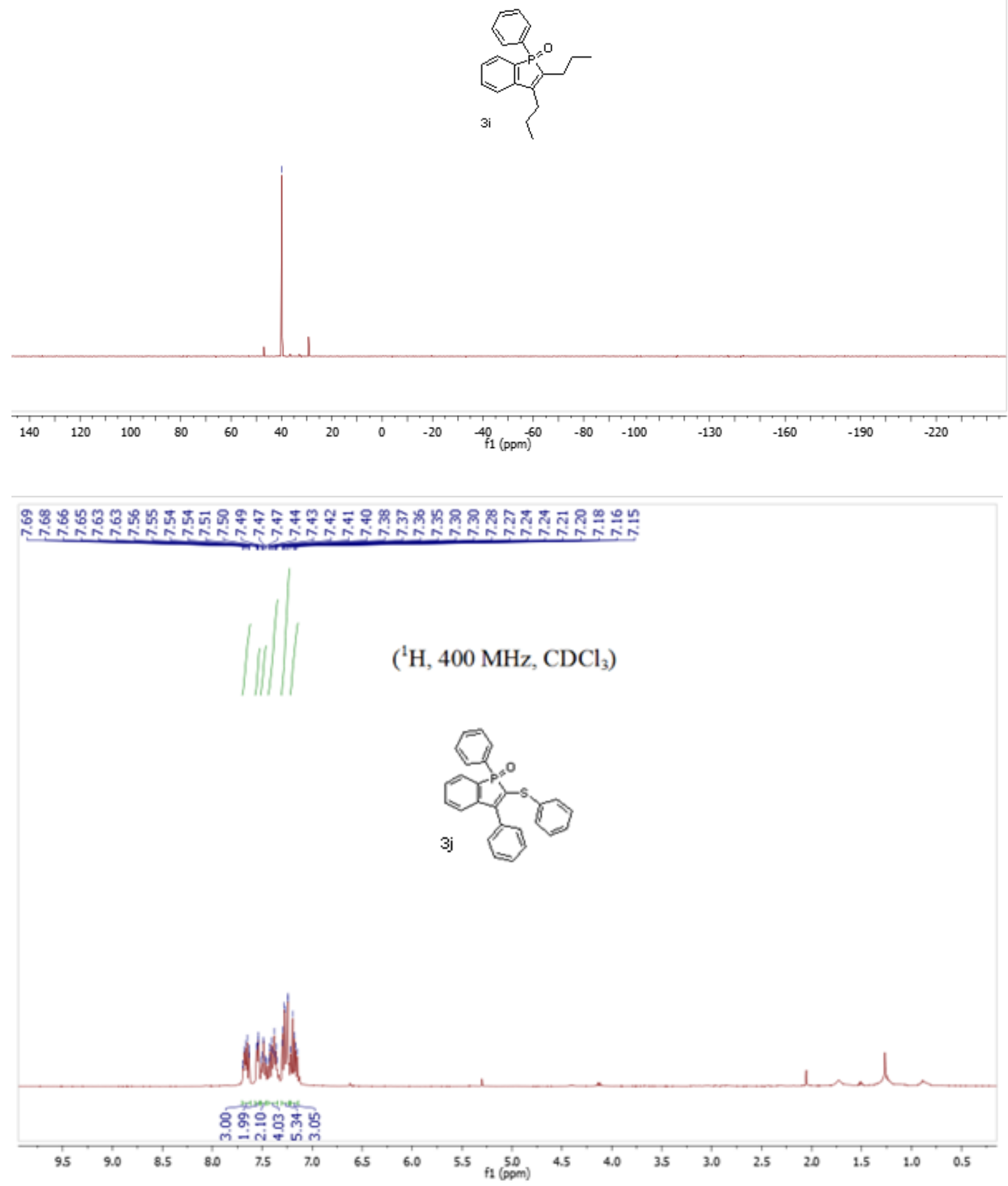
$\left({ }^{13} \mathrm{C}, 100 \mathrm{MHz}, \mathrm{CDCl}_{3}\right)$

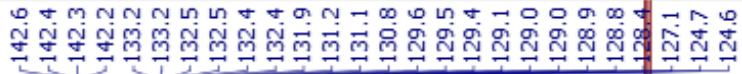
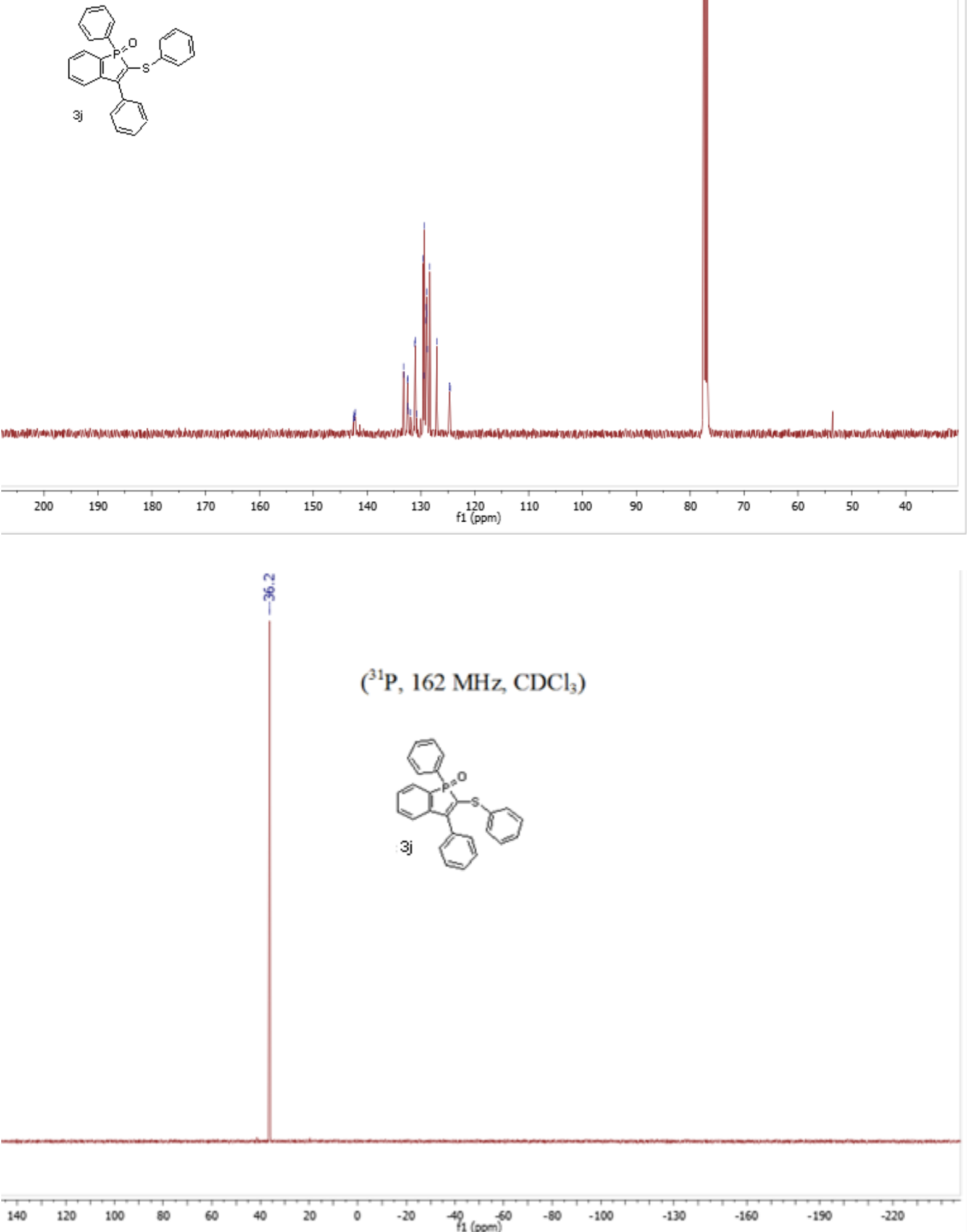

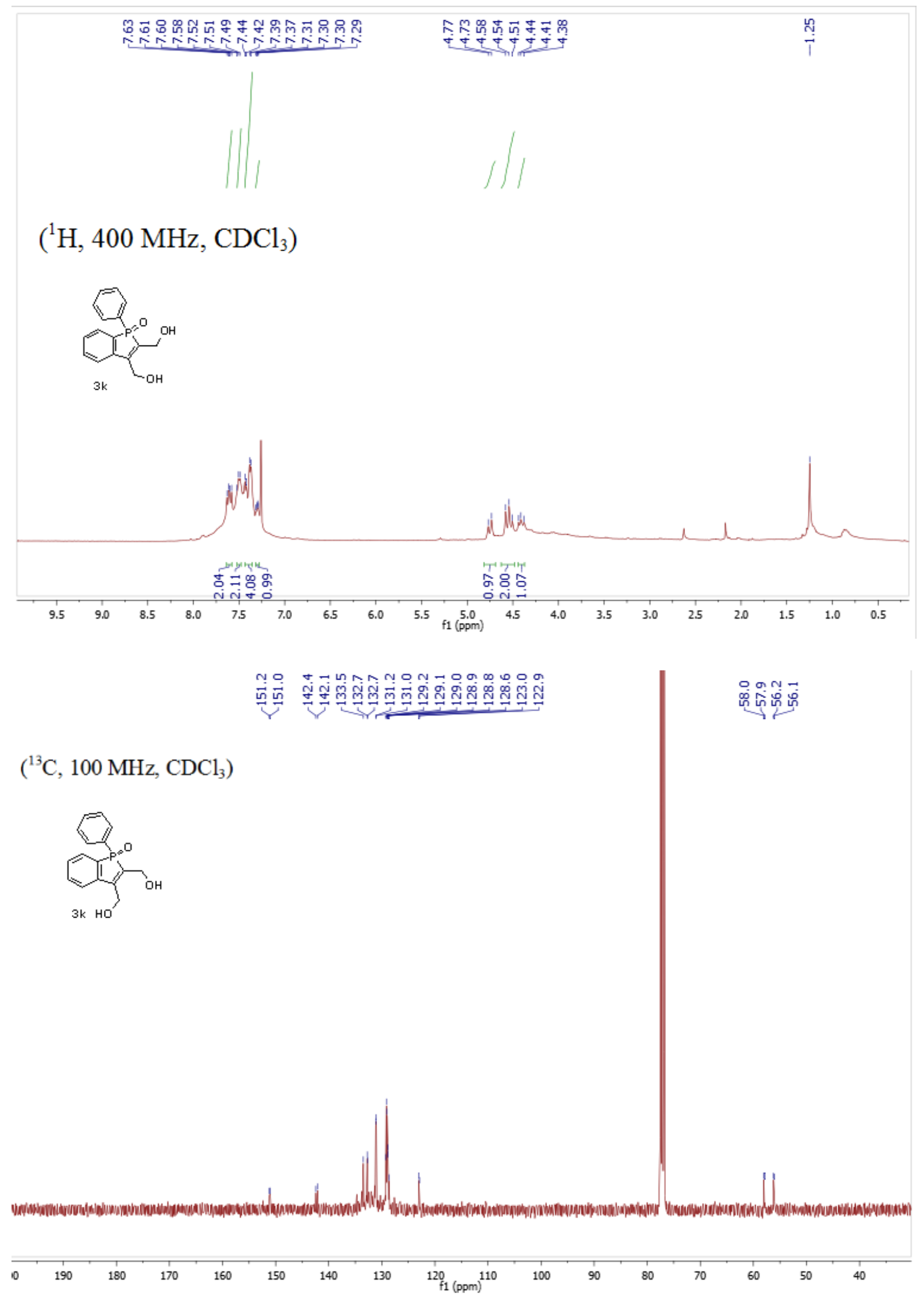
$\left({ }^{31} \mathrm{P}, 162 \mathrm{MHz}, \mathrm{CDCl}_{3}\right)$

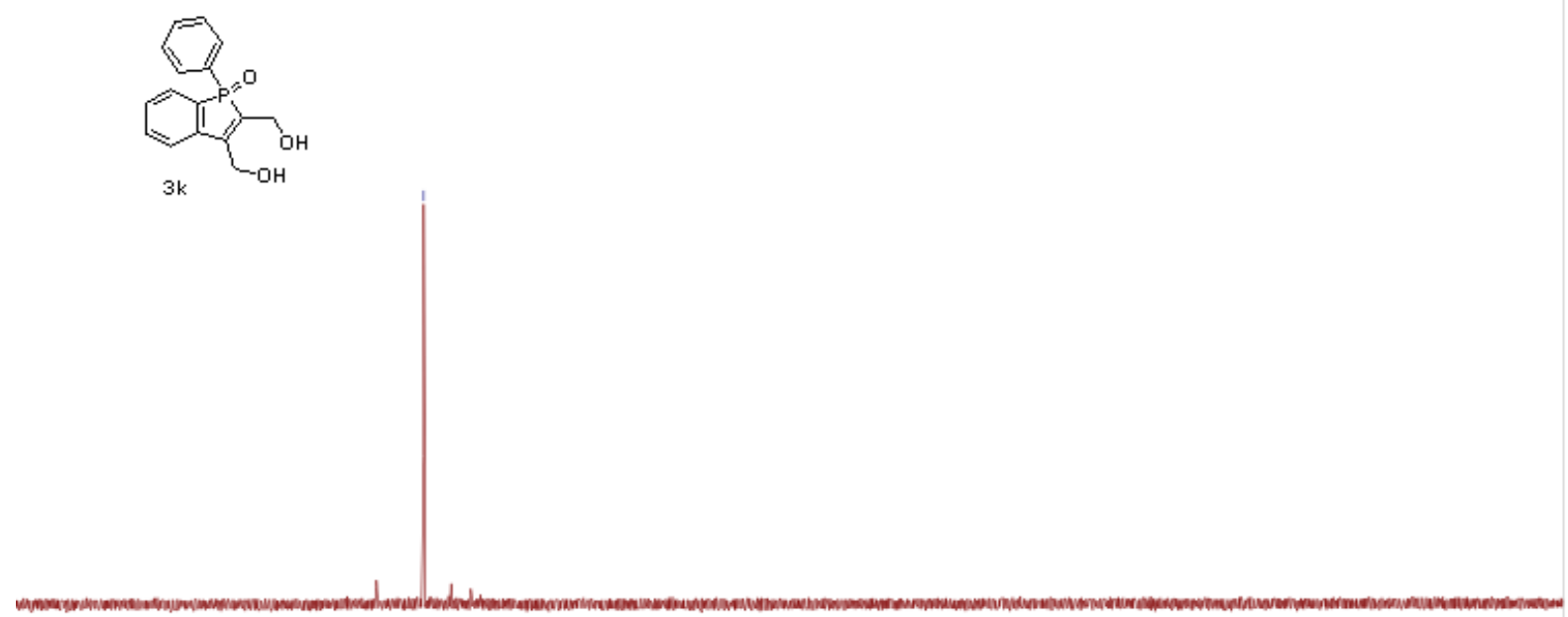

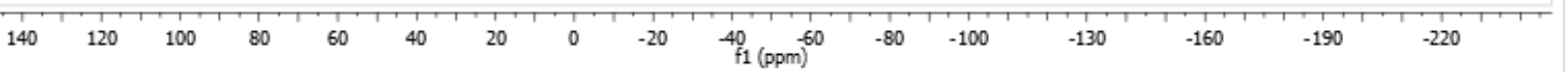

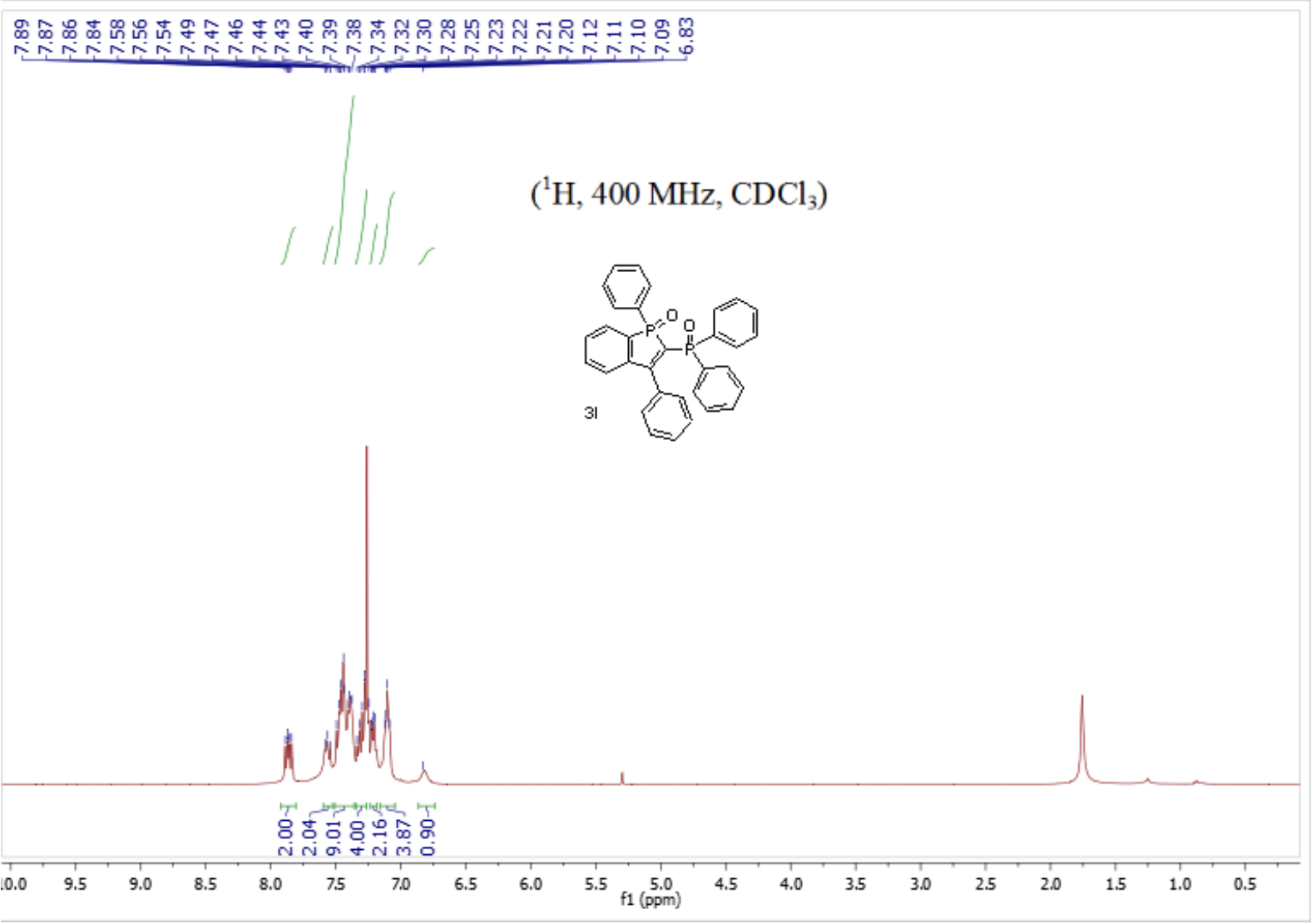




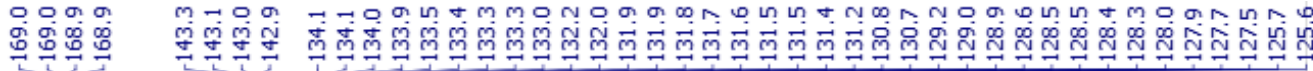

$\left({ }^{13} \mathrm{C}, 100 \mathrm{MHz}, \mathrm{CDCl}_{3}\right)$
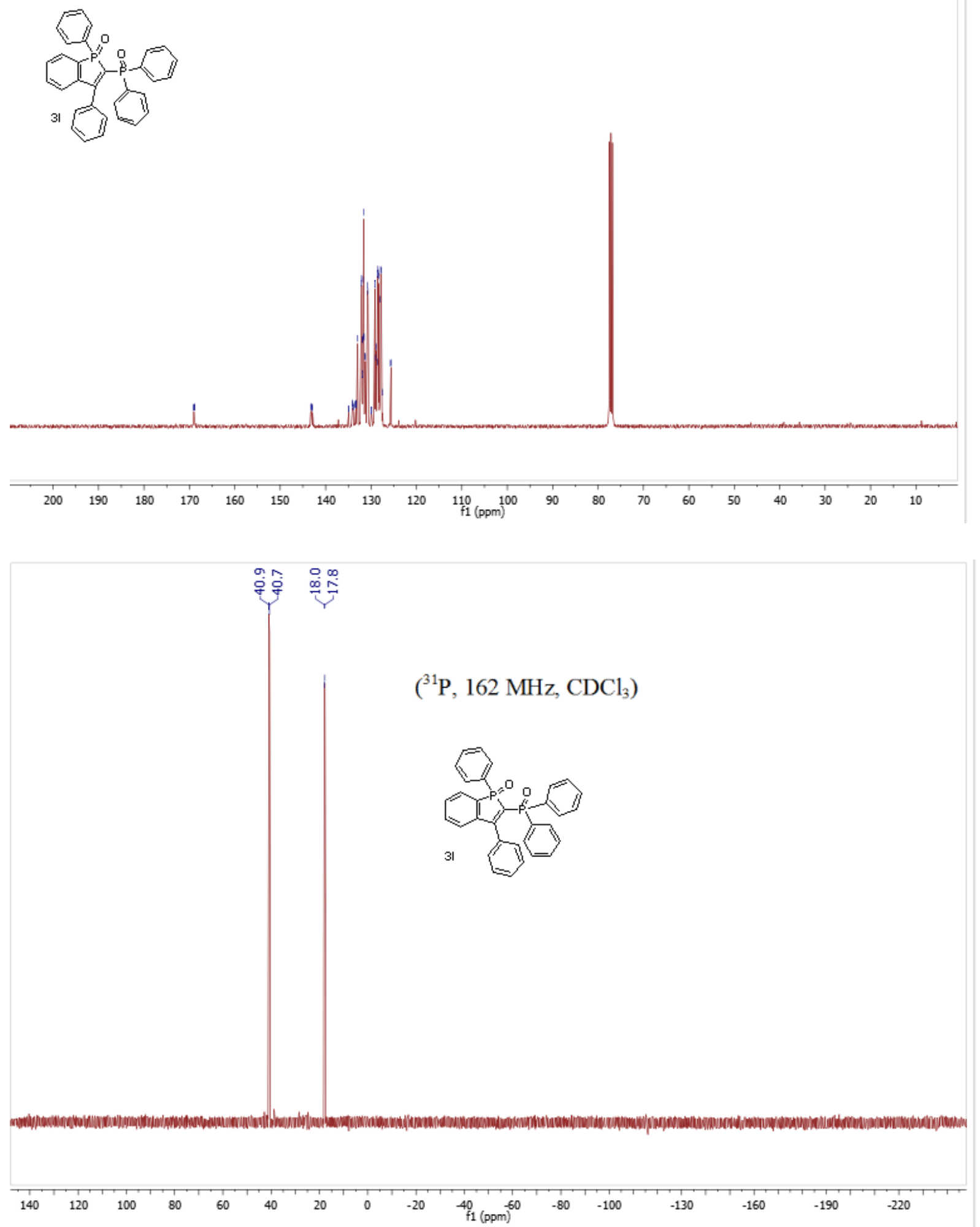


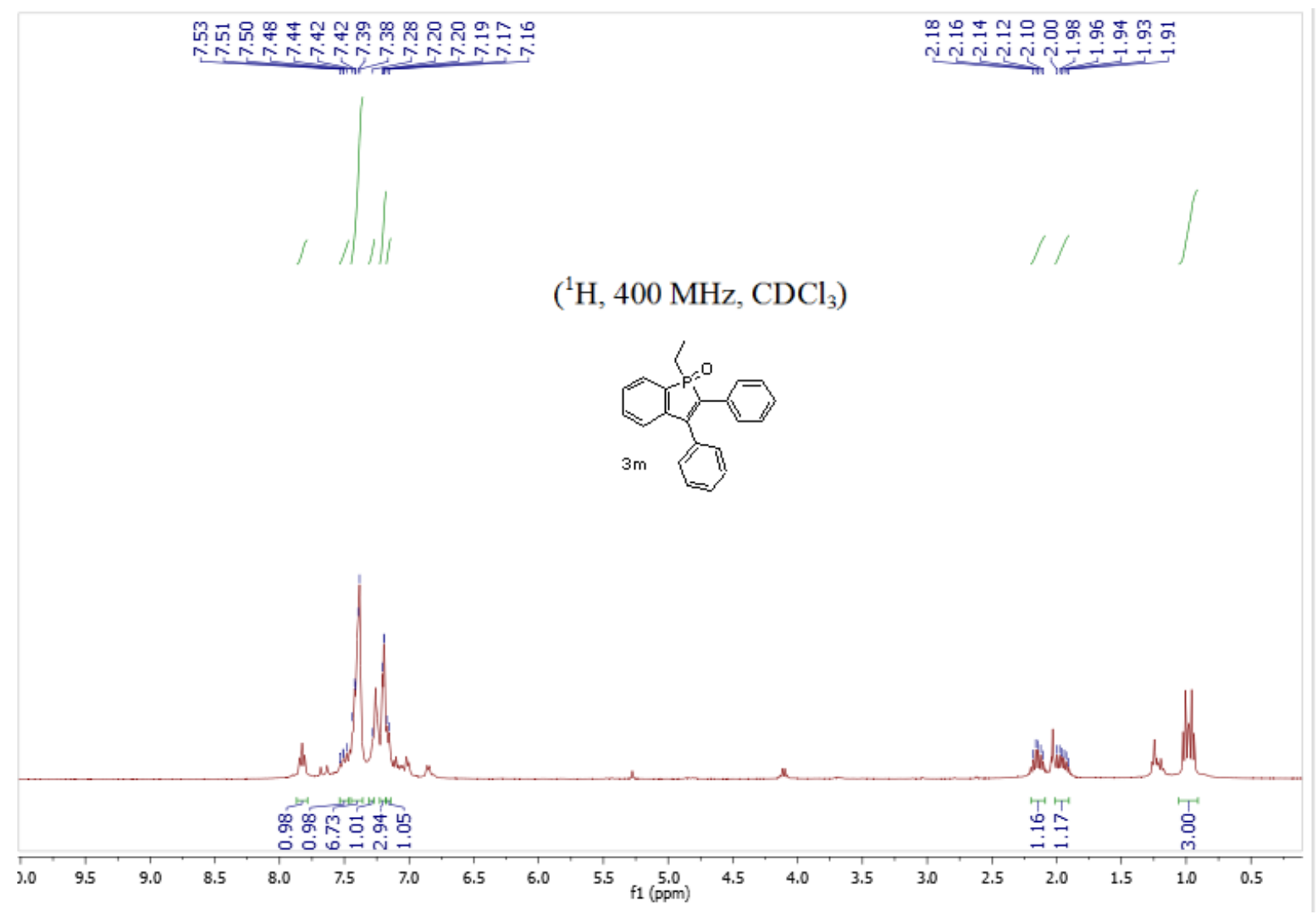

$\left({ }^{13} \mathrm{C}, 100 \mathrm{MHz}, \mathrm{CDCl}_{3}\right)$
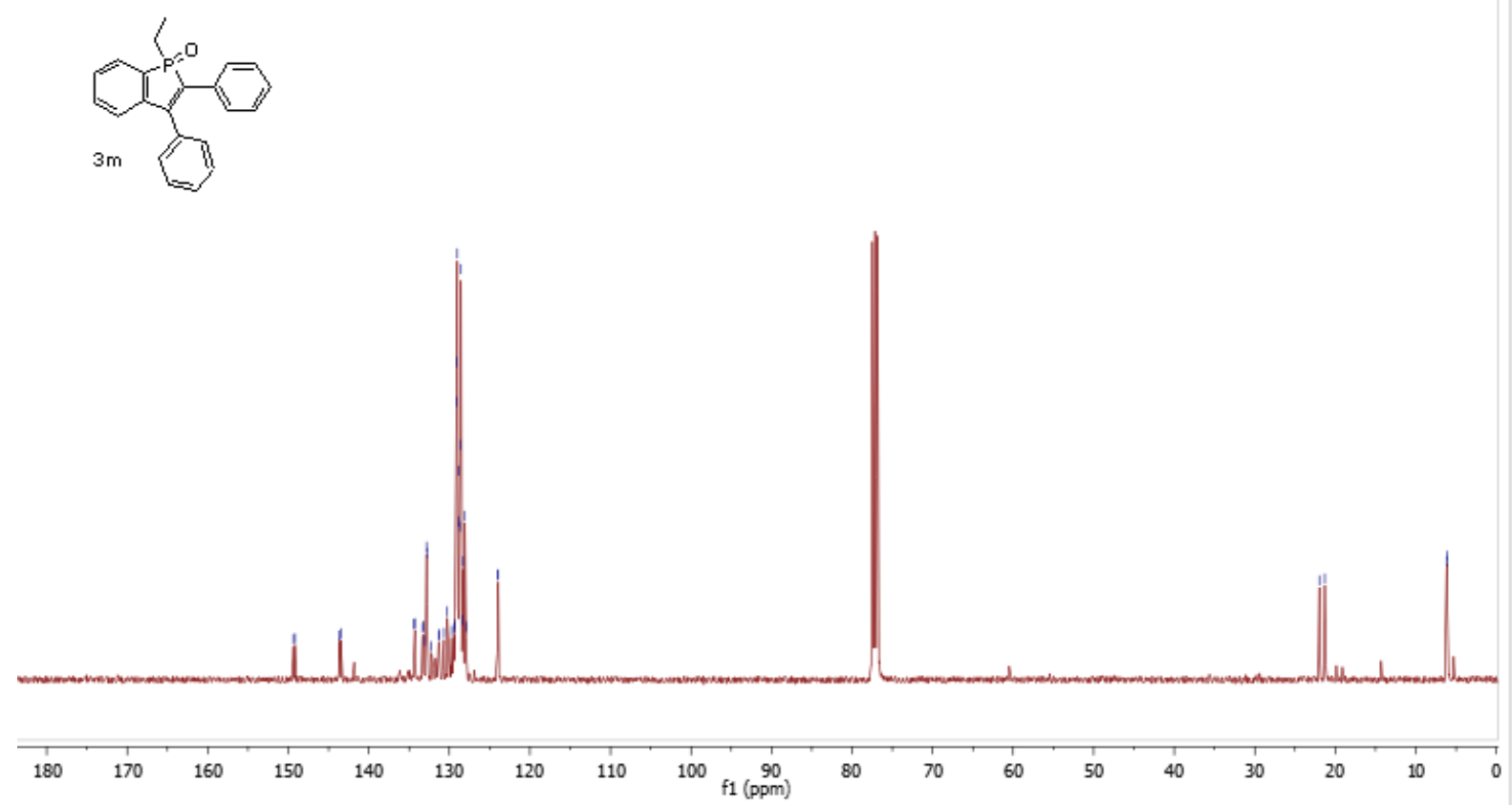
$\left({ }^{31} \mathrm{P}, 162 \mathrm{MHz}, \mathrm{CDCl}_{3}\right)$

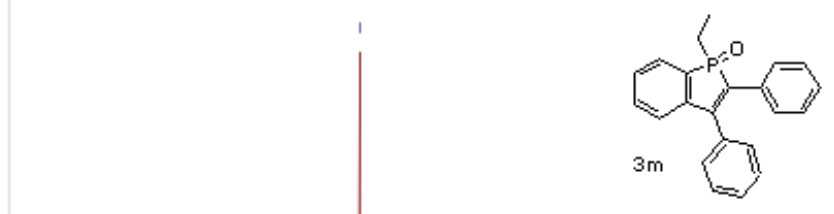

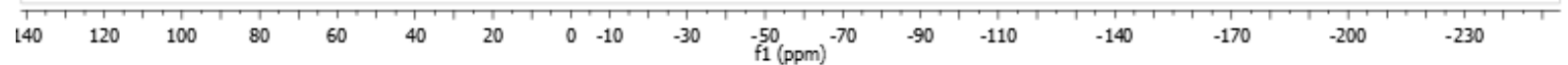

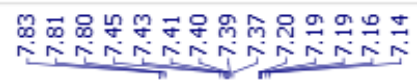

$\left({ }^{1} \mathrm{H}, 400 \mathrm{MHz}, \mathrm{CDCl}_{3}\right)$
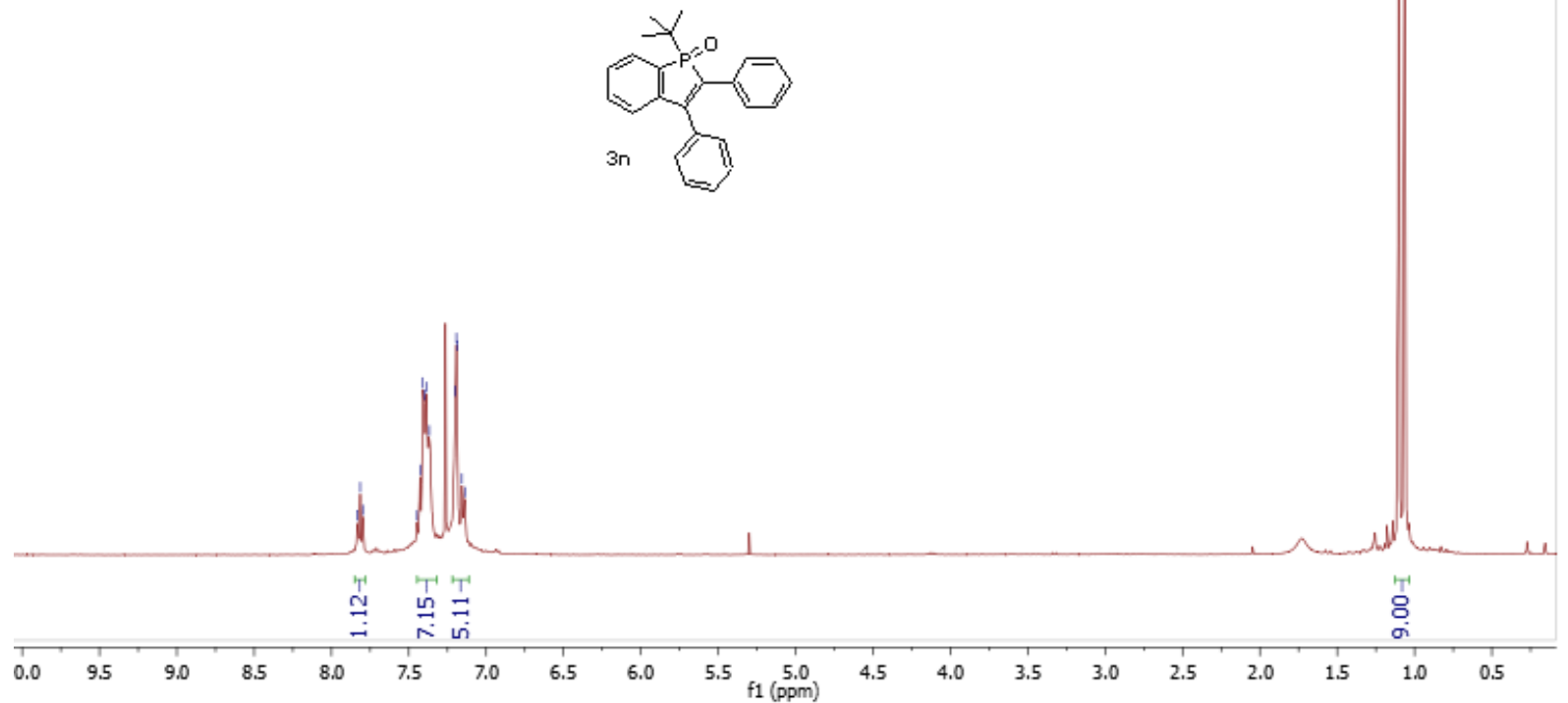

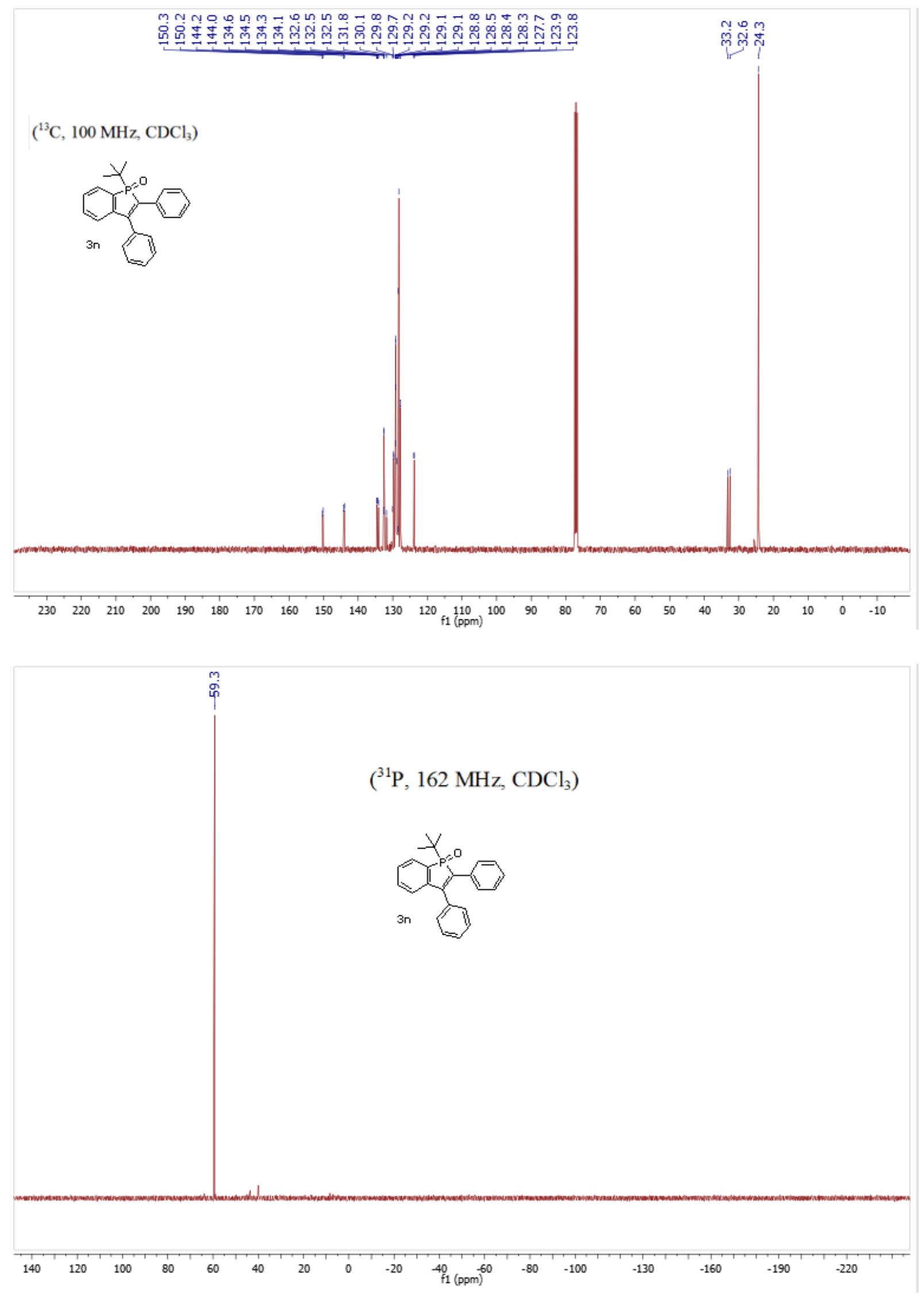


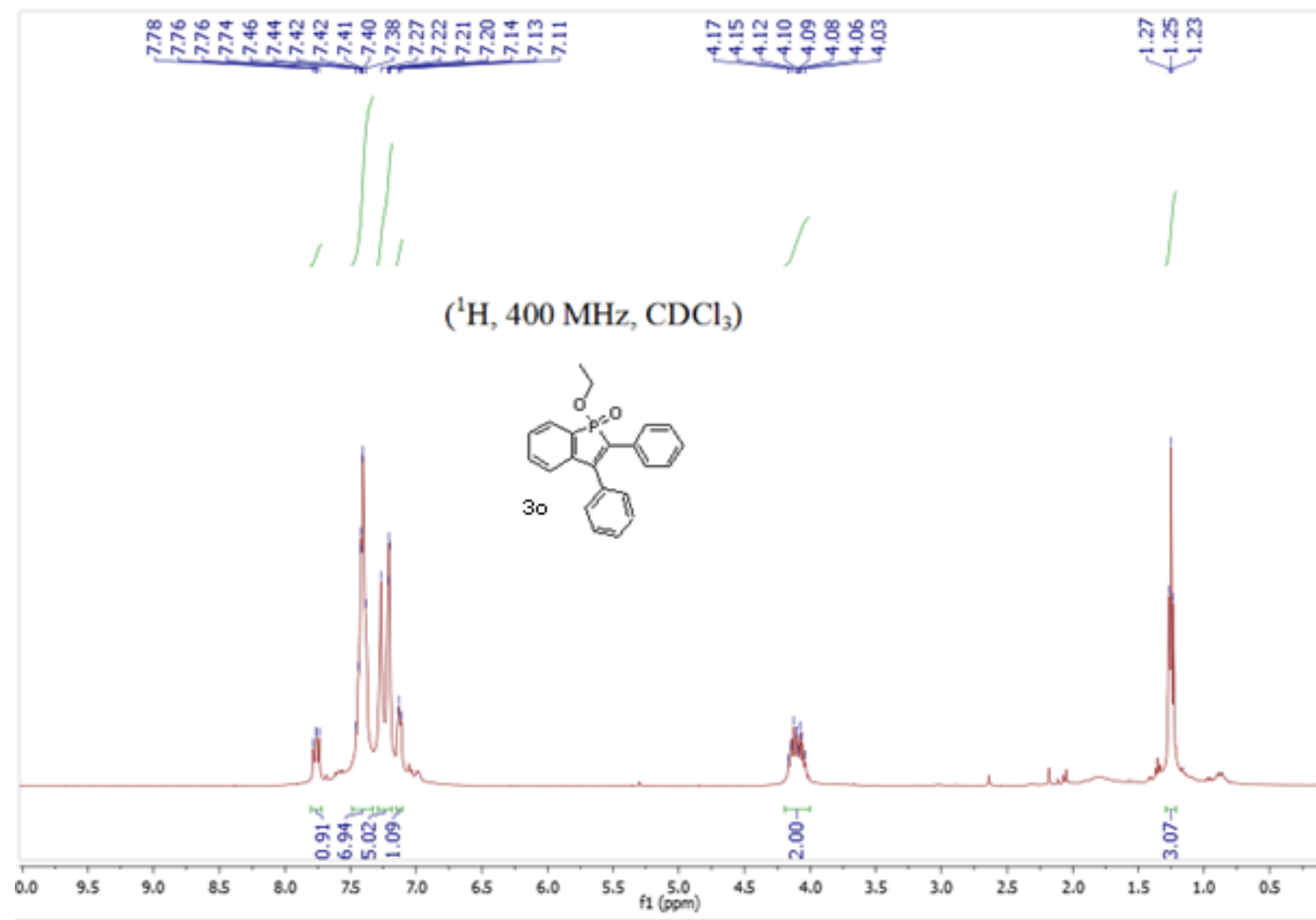

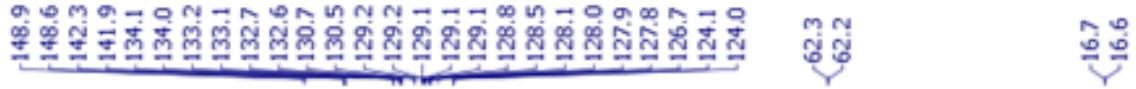

$\left({ }^{13} \mathrm{C}, 100 \mathrm{MHz}, \mathrm{CDCl}_{3}\right)$

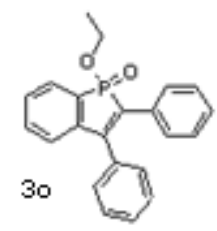

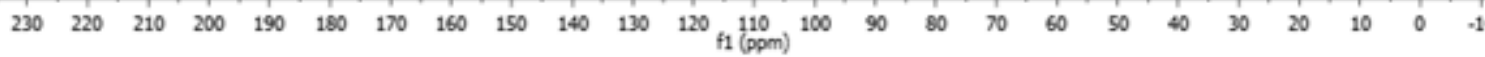


$\stackrel{9}{\text { ? }}$

$\left({ }^{31} \mathrm{P}, 162 \mathrm{MHz}, \mathrm{CDCl}_{3}\right)$

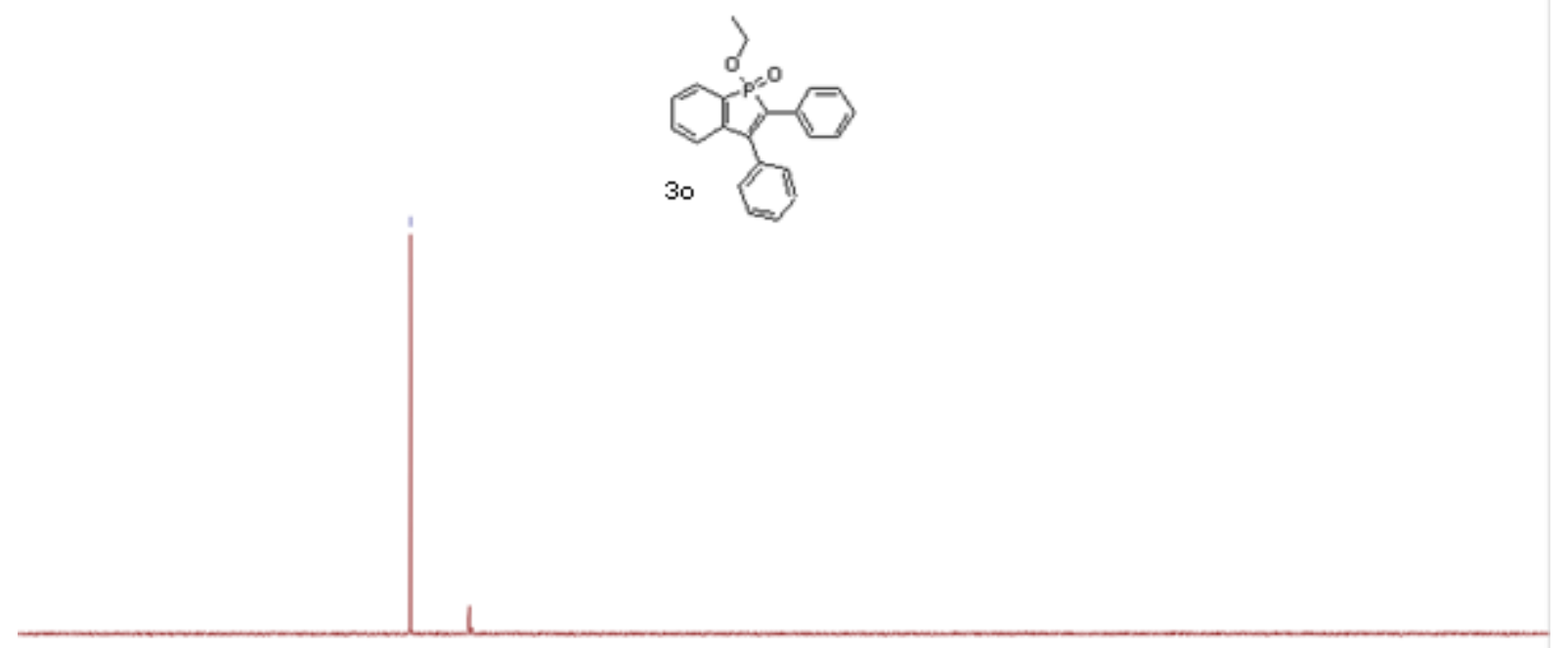

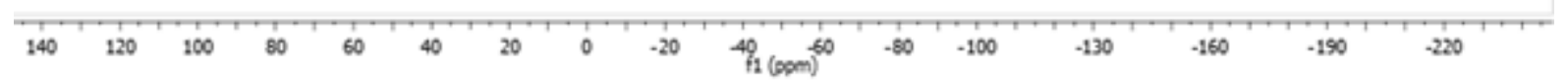

$\left({ }^{\mathrm{H}} \mathrm{H}, 400 \mathrm{MHz}, \mathrm{CDCl}_{3}\right)$

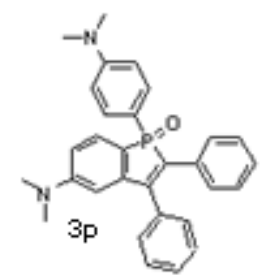

child k

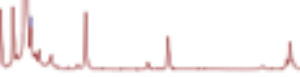

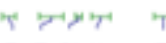

'T'

ริํํำ 욤

$\stackrel{8}{\mathrm{i}}$

$\begin{array}{llll}9.5 & 9.0 & 8.5 & 8.0\end{array}$

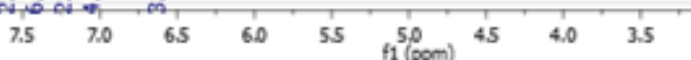

i 
$\left({ }^{13} \mathrm{C}, 100 \mathrm{MHz}, \mathrm{CDCl}_{3}\right)$

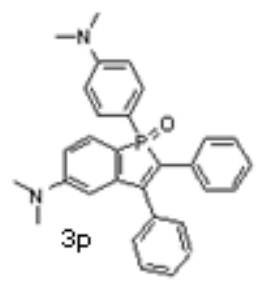

$\bar{i}$

$\left({ }^{31} \mathrm{P}, 162 \mathrm{MHz}, \mathrm{CDCl}_{3}\right)$
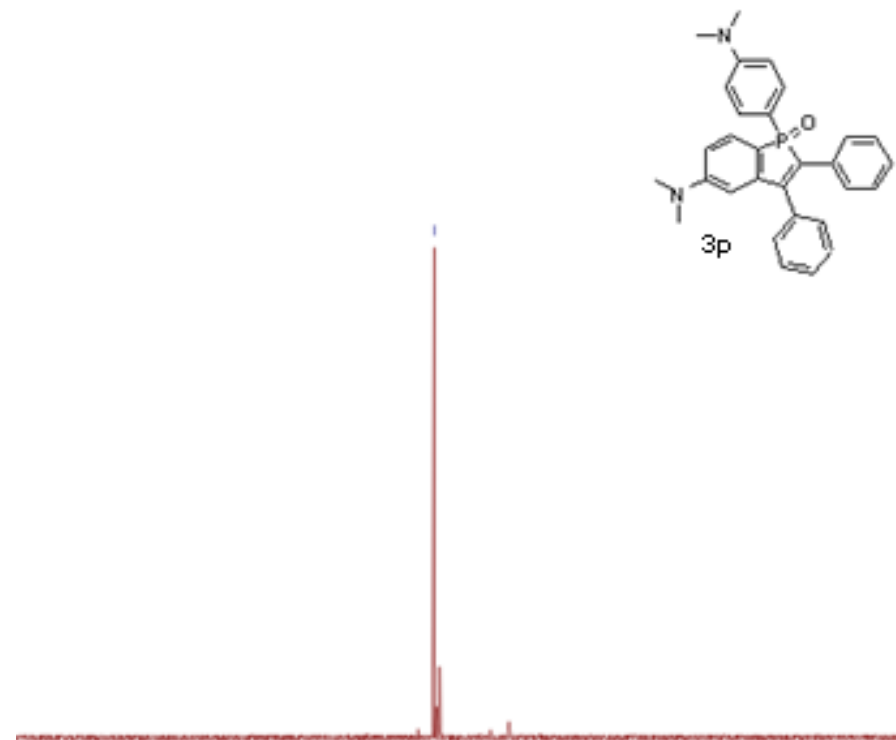

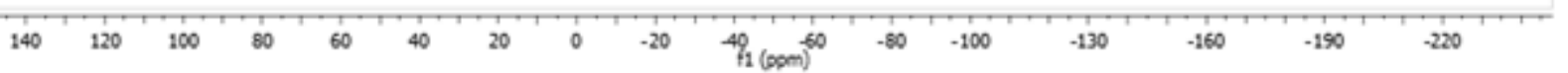




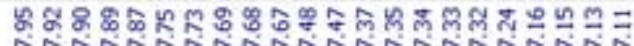

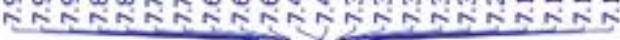

$\left({ }^{1} \mathrm{H}, 400 \mathrm{MHz}, \mathrm{CDCl}_{3}\right)$
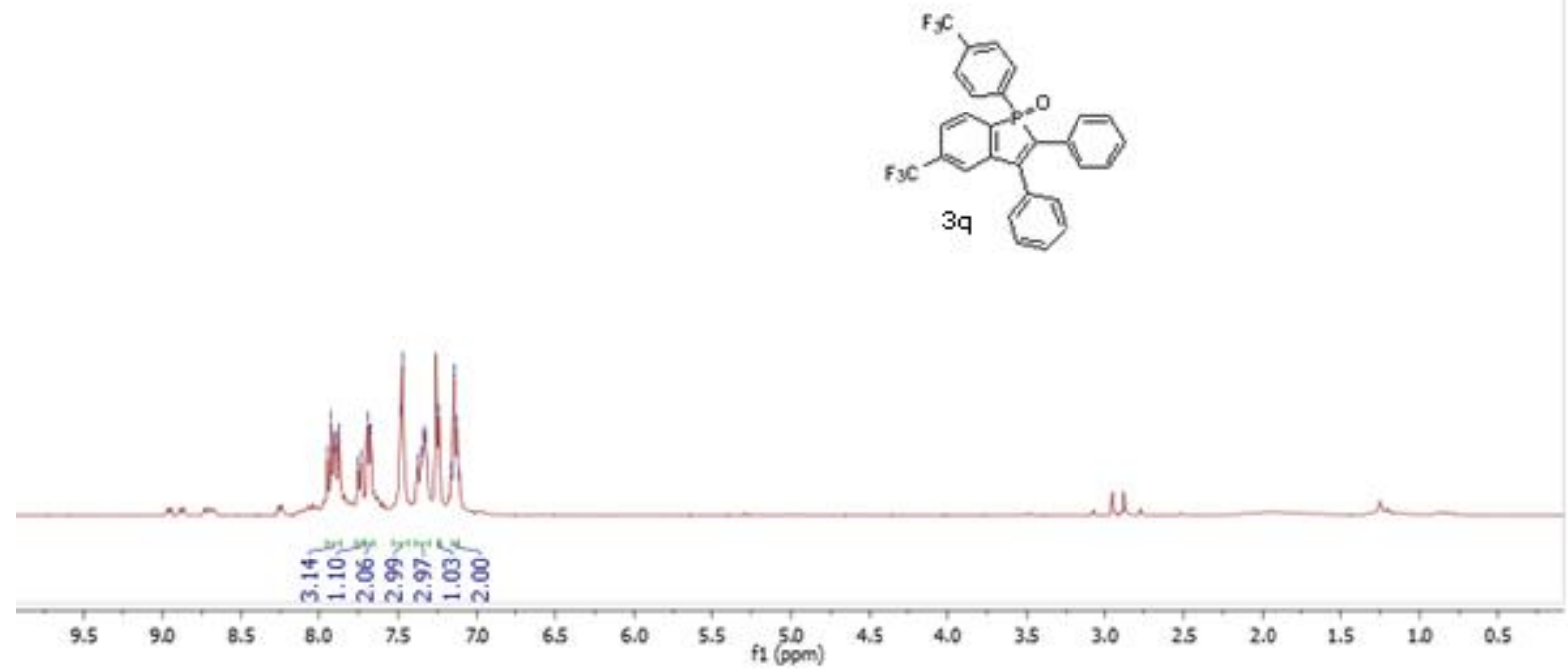

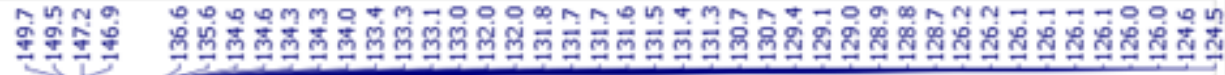

$\left({ }^{13} \mathrm{C}, 100 \mathrm{MHz}, \mathrm{CDCl}_{3}\right)$
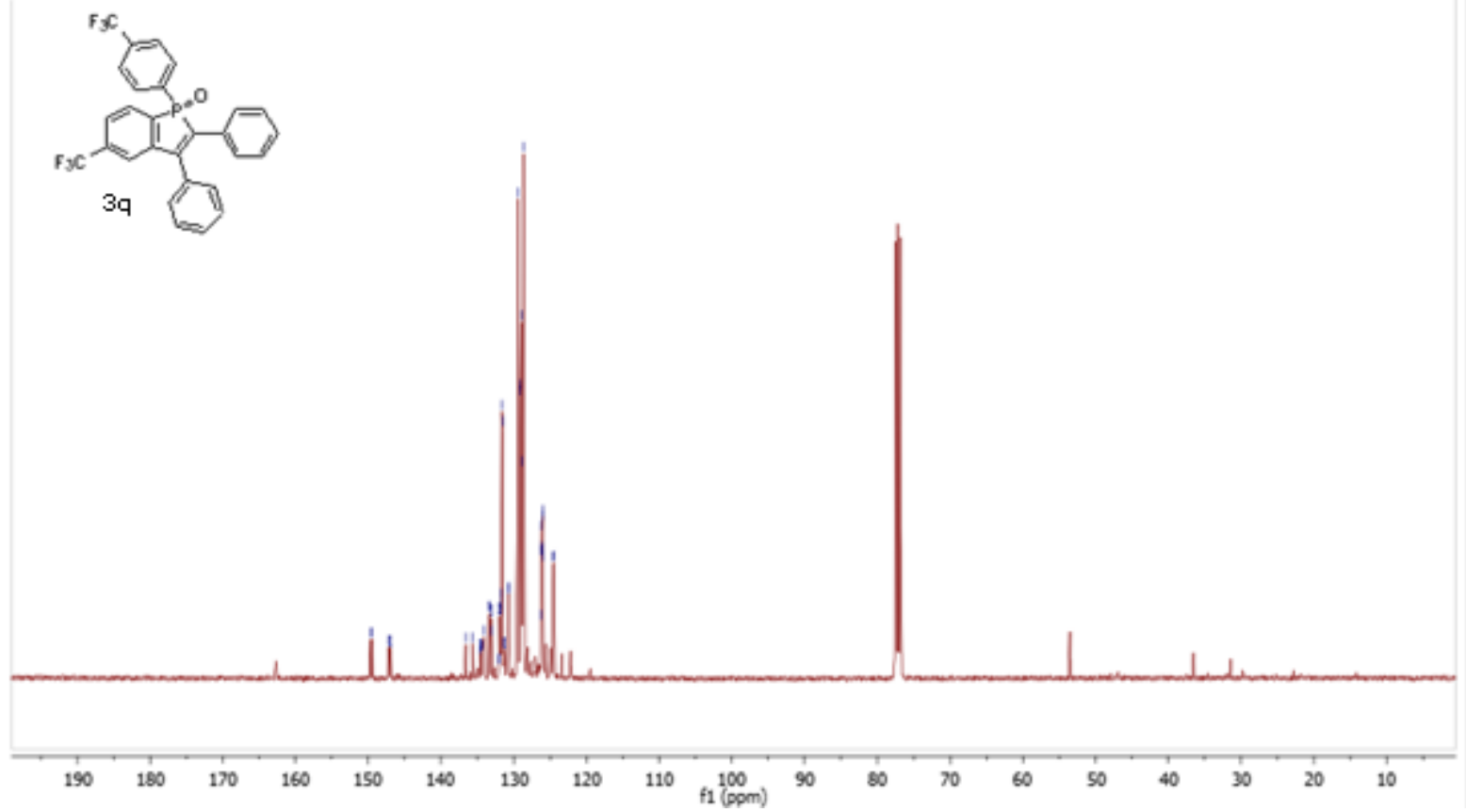
审

$\left({ }^{31} \mathrm{P}, 162 \mathrm{MHz}, \mathrm{CDCl}_{3}\right)$
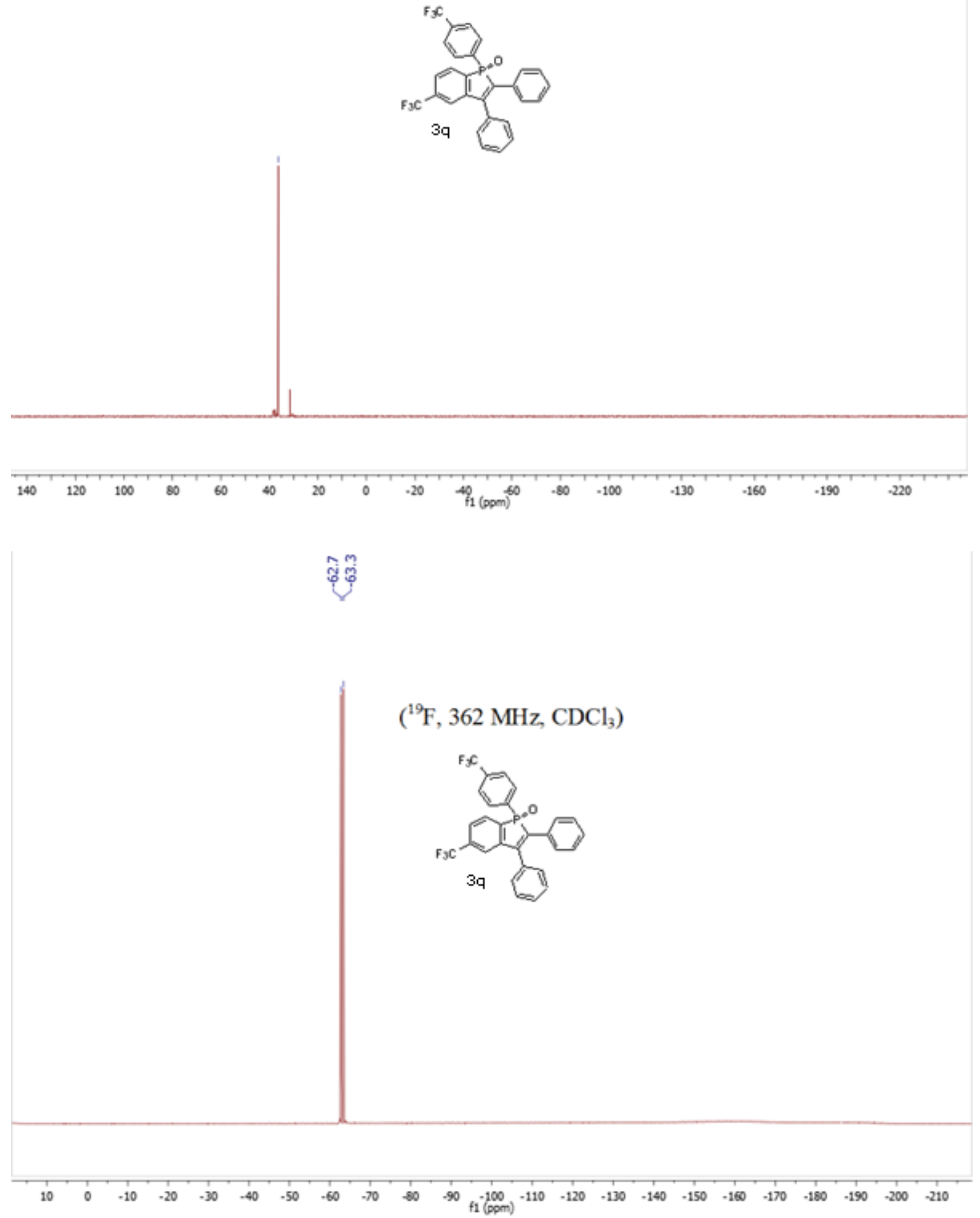

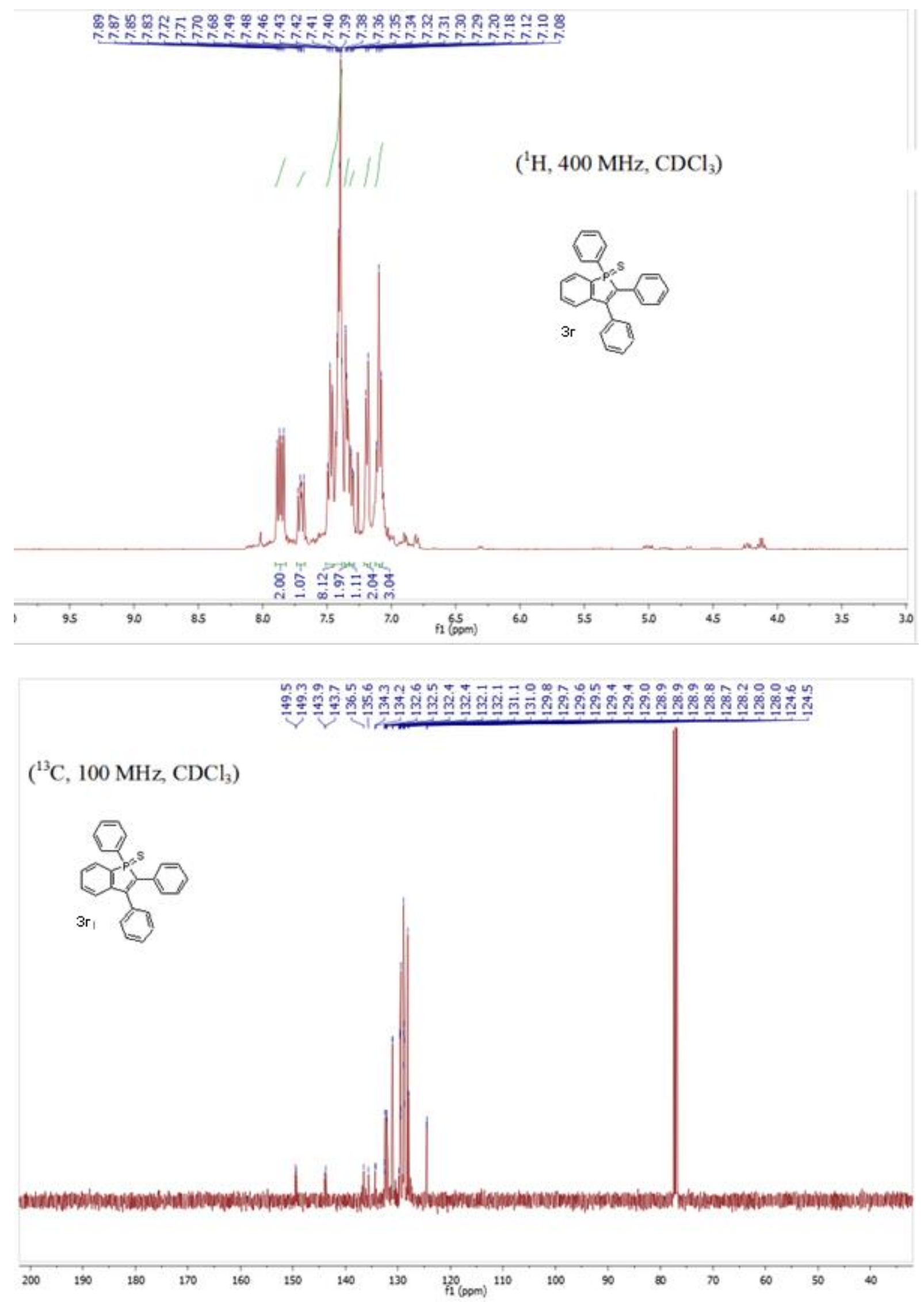
$\left({ }^{31} \mathrm{P}, 162 \mathrm{MHz}, \mathrm{CDCl}_{3}\right)$
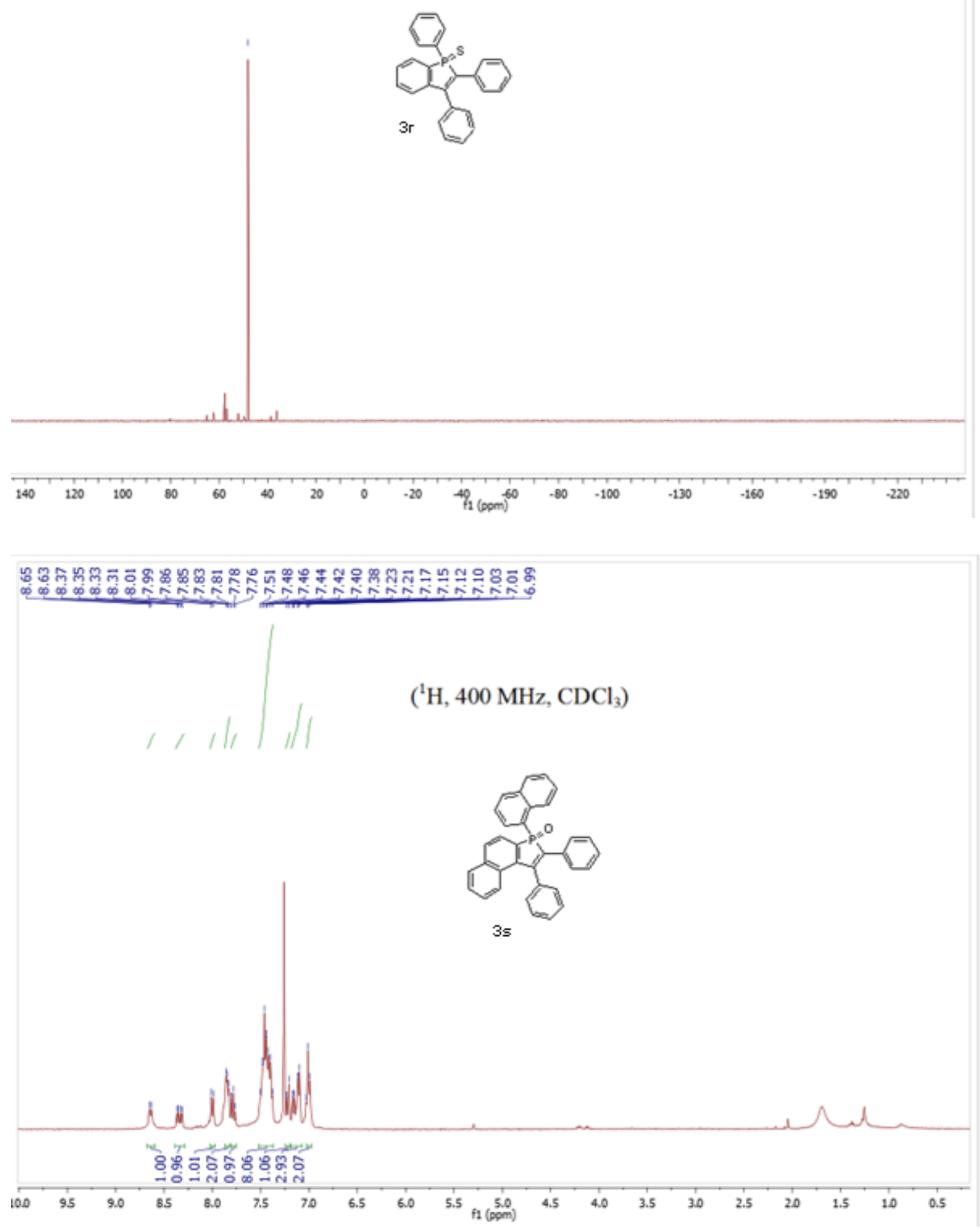


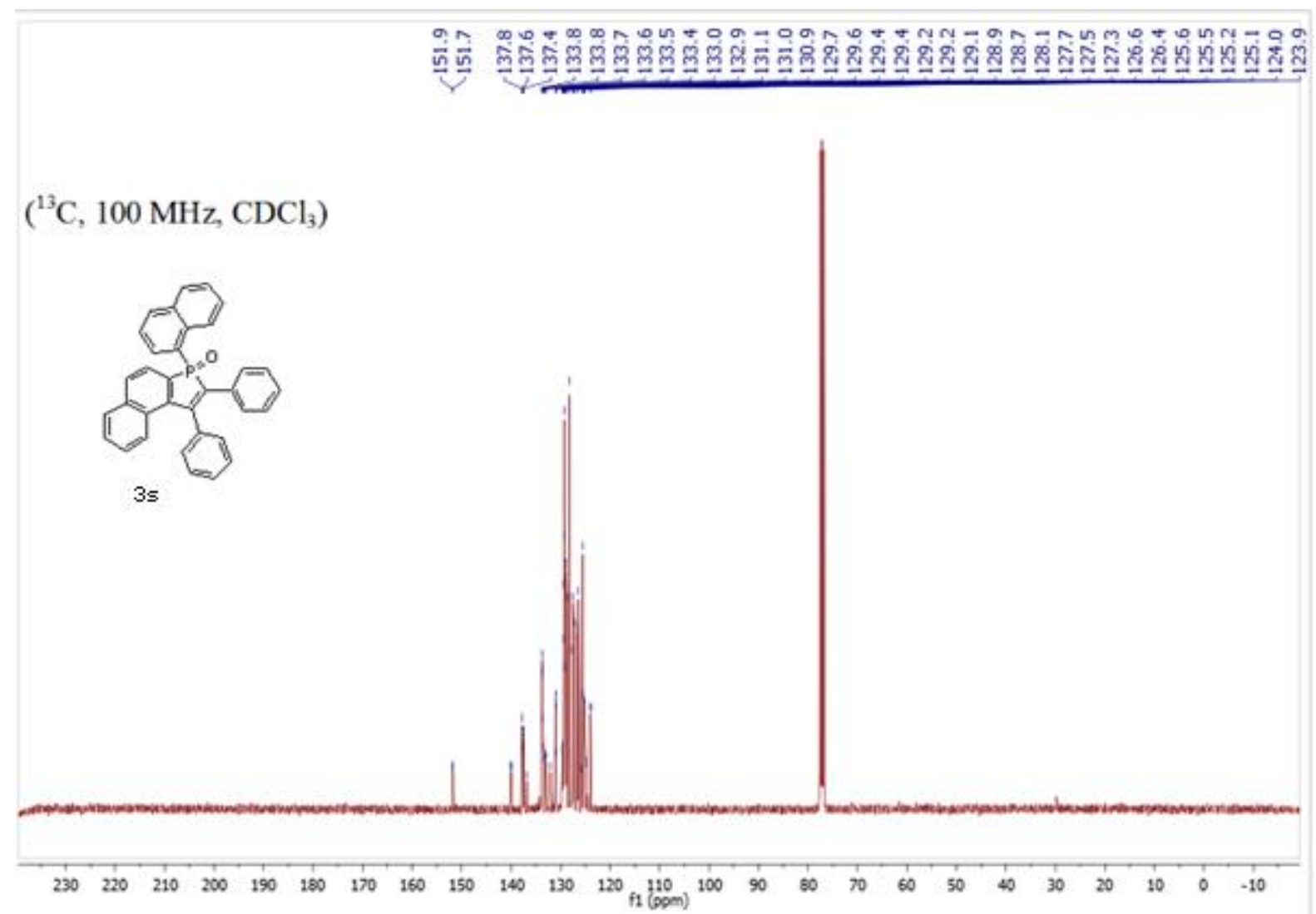

$$
\stackrel{\circ}{\varphi}
$$

$\left({ }^{31} \mathrm{P}, 162 \mathrm{MHz}, \mathrm{CDCl}_{3}\right)$

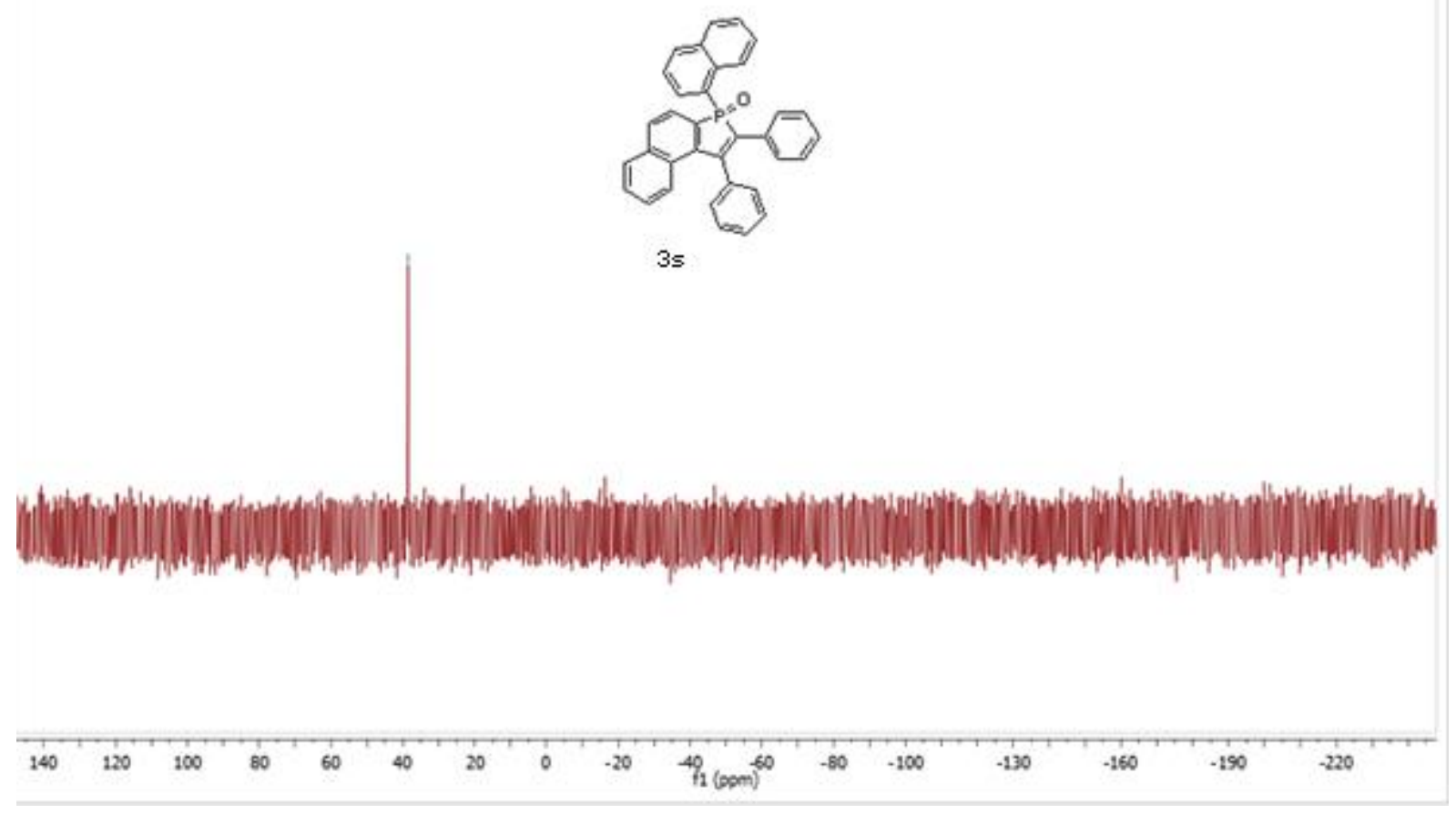



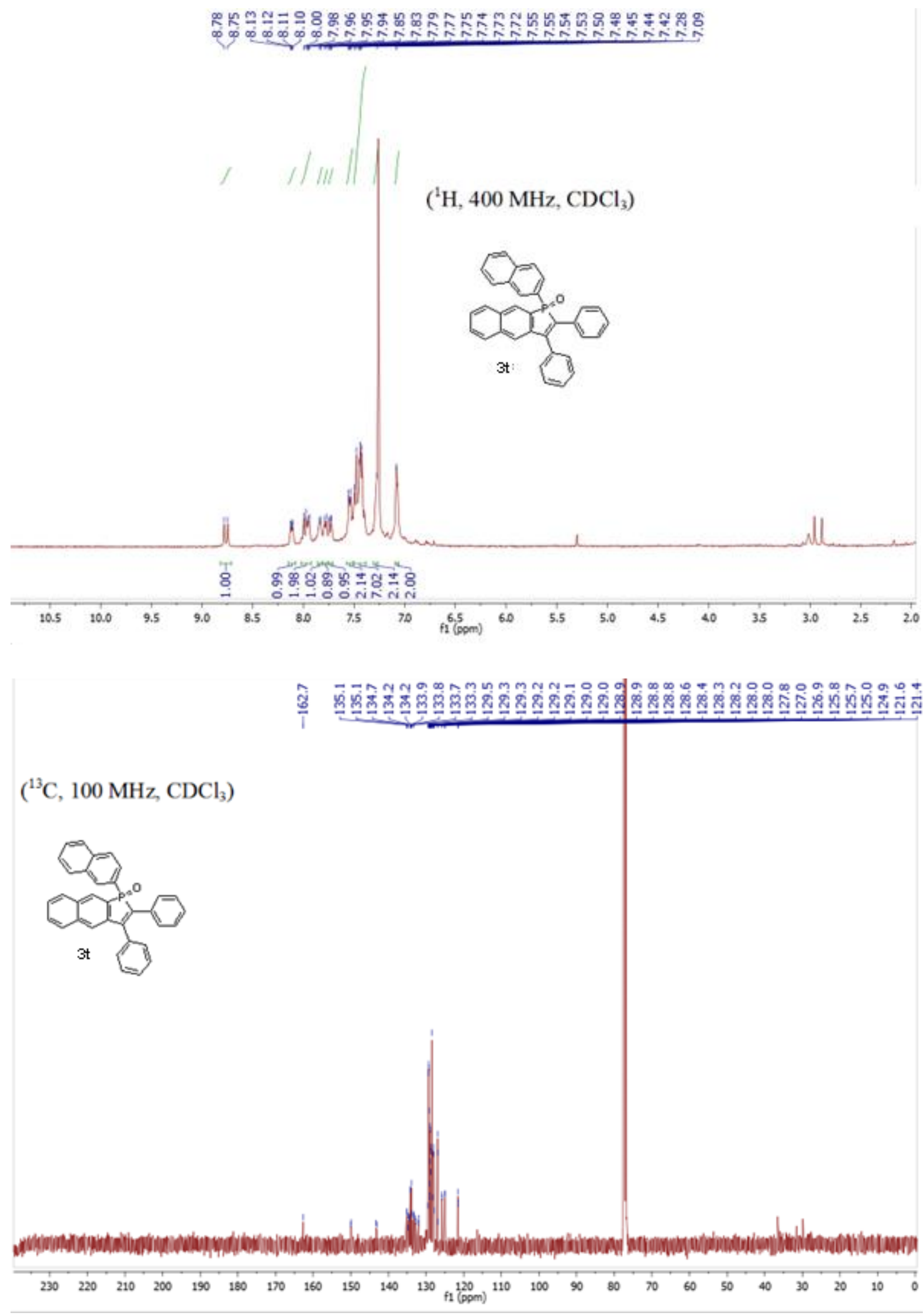


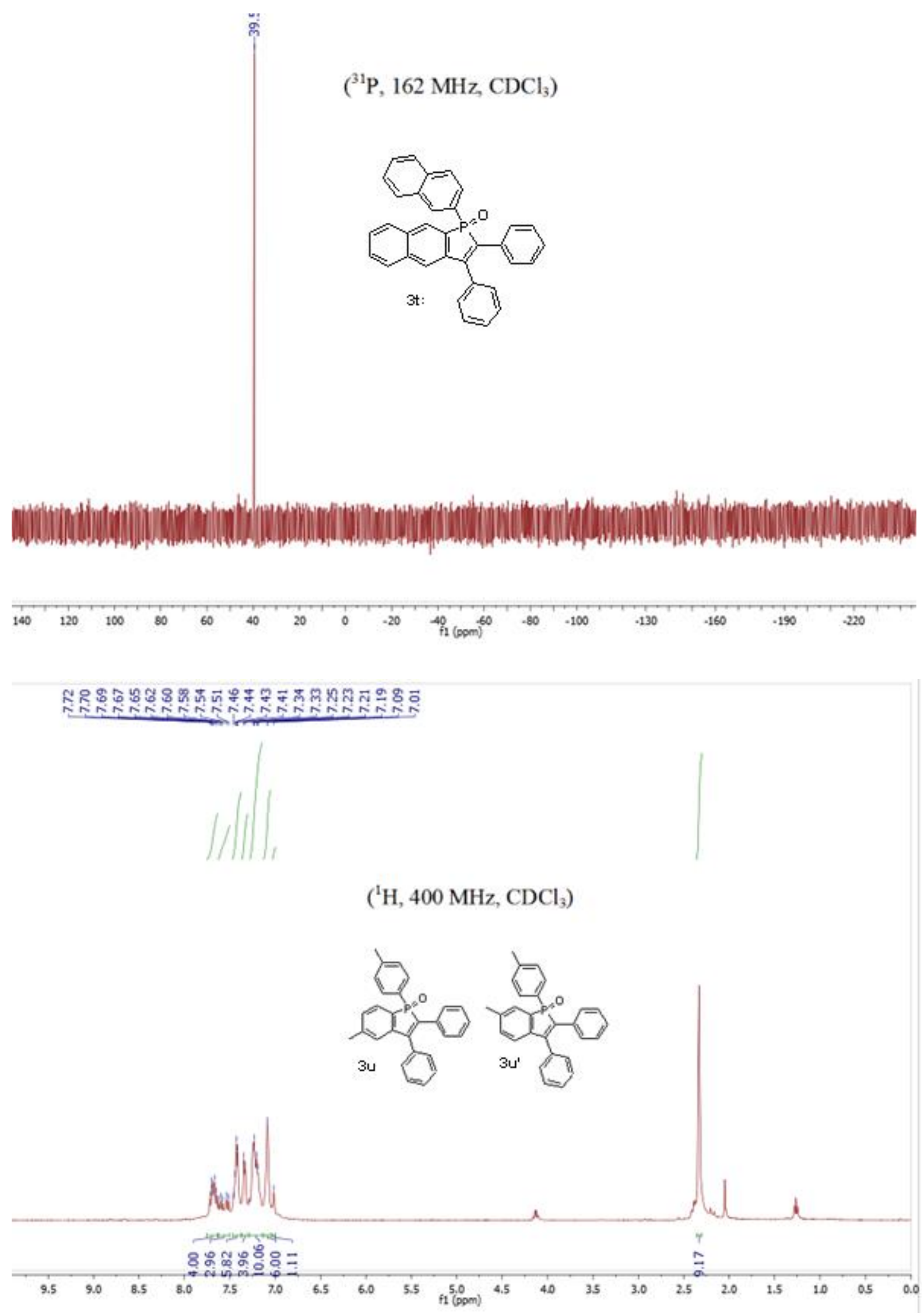


$\left({ }^{13} \mathrm{C}, 100 \mathrm{MHz}, \mathrm{CDCl}_{3}\right)$
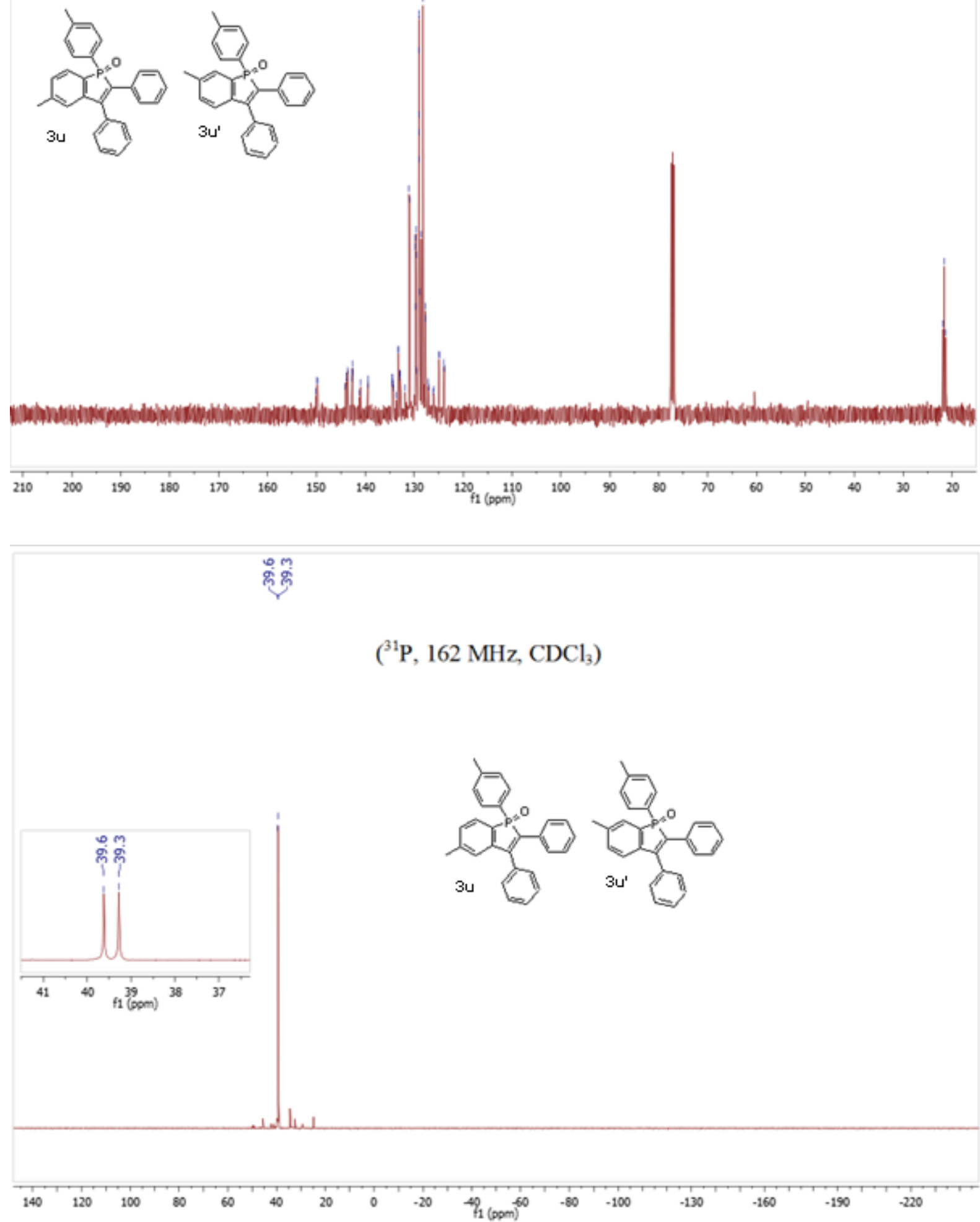


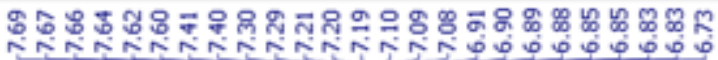

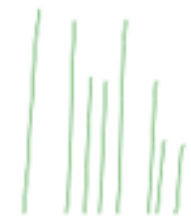

$\left({ }^{1} \mathrm{H}, 400 \mathrm{MHz}, \mathrm{CDCl}_{3}\right)$
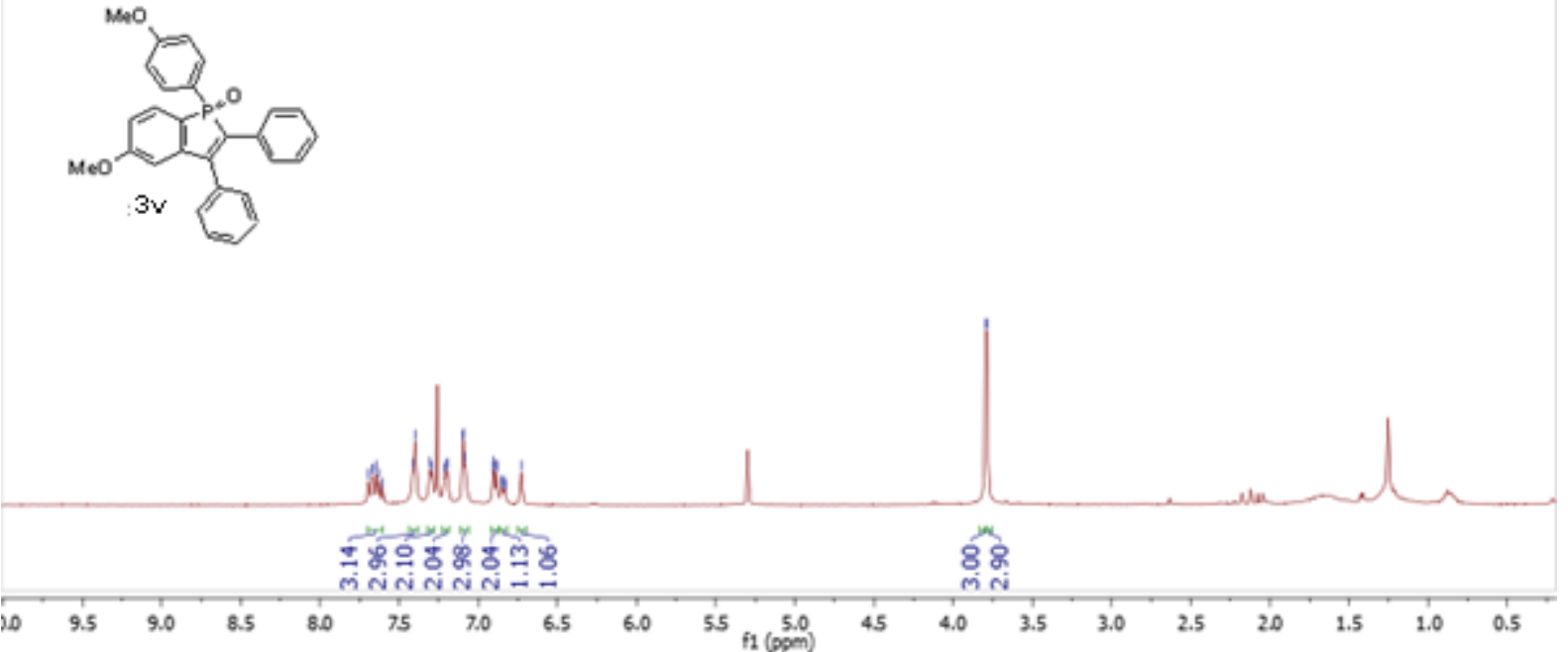

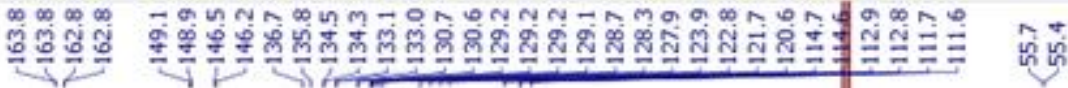

$\left({ }^{13} \mathrm{C}, 100 \mathrm{MHz}, \mathrm{CDCl}_{3}\right)$
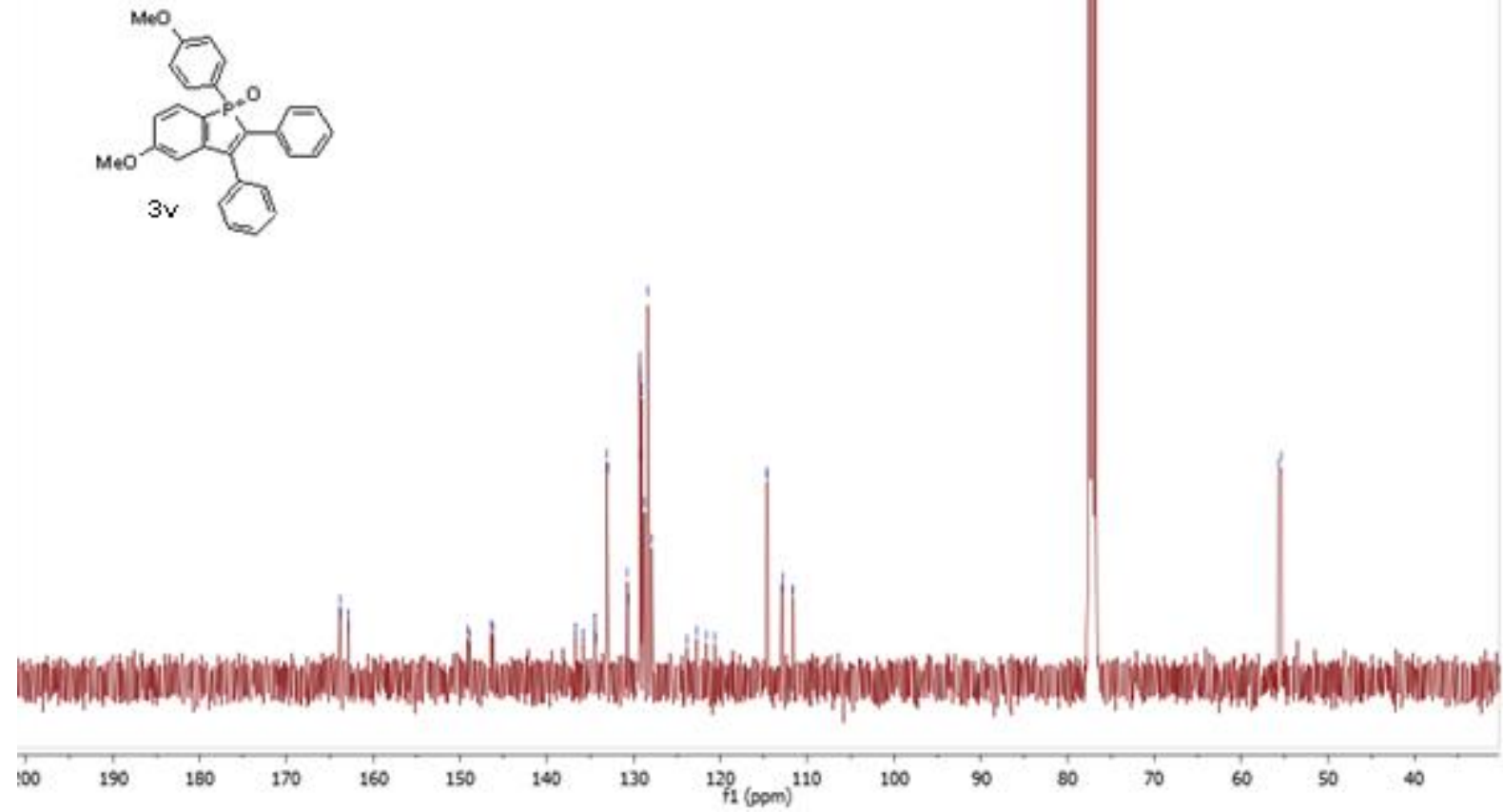
$\overrightarrow{\text { p }}$

$\left({ }^{31} \mathrm{P}, 162 \mathrm{MHz}, \mathrm{CDCl}_{3}\right)$
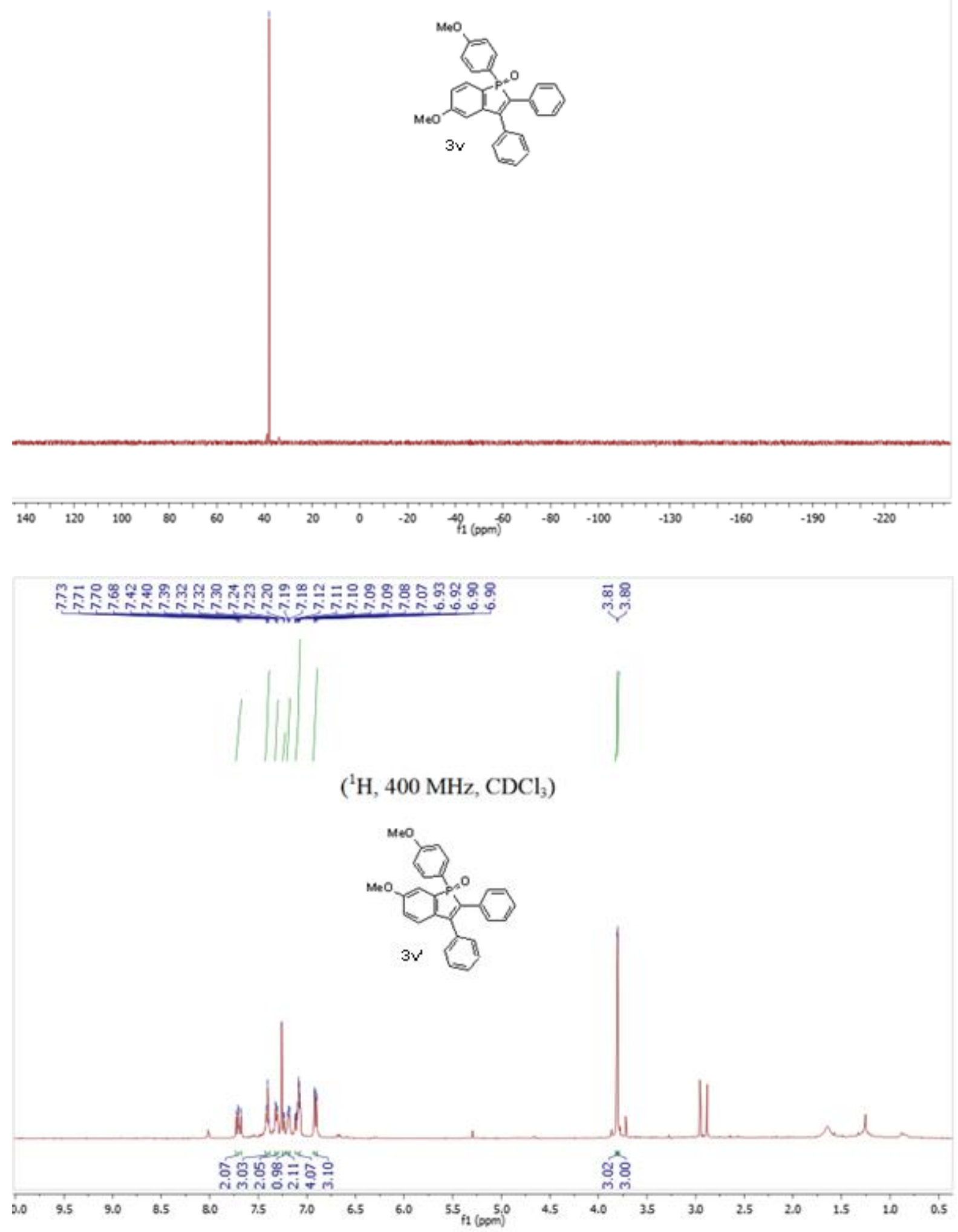


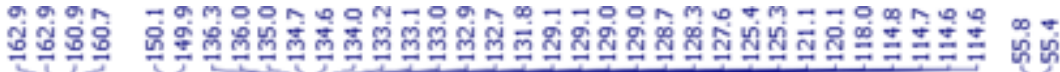

$\left({ }^{13} \mathrm{C}, 100 \mathrm{MHz}, \mathrm{CDCl}_{3}\right)$
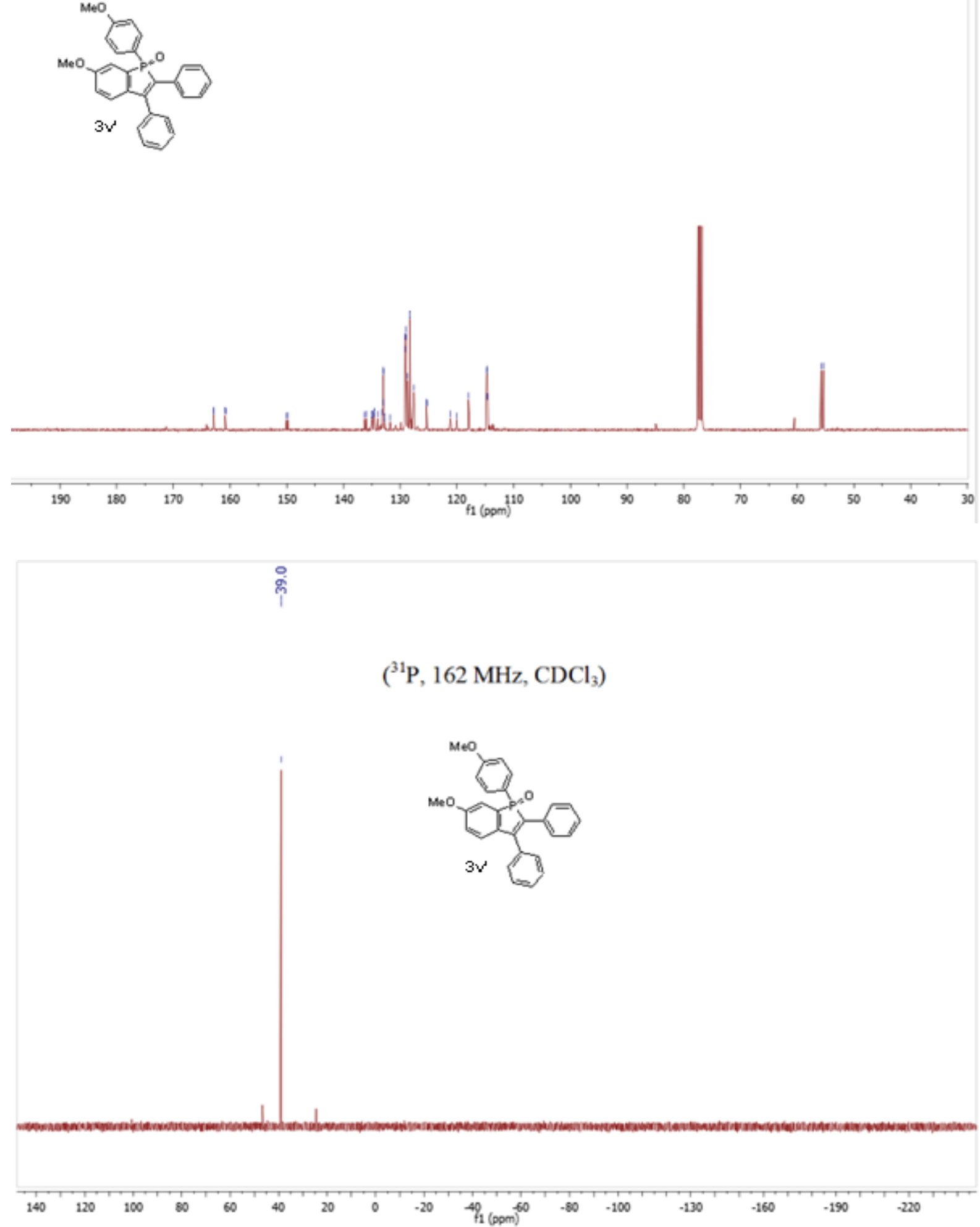


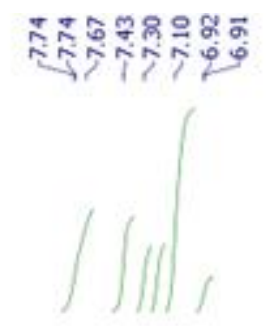

$\left({ }^{1} \mathrm{H}, 400 \mathrm{MHz}, \mathrm{CDCl}_{3}\right)$
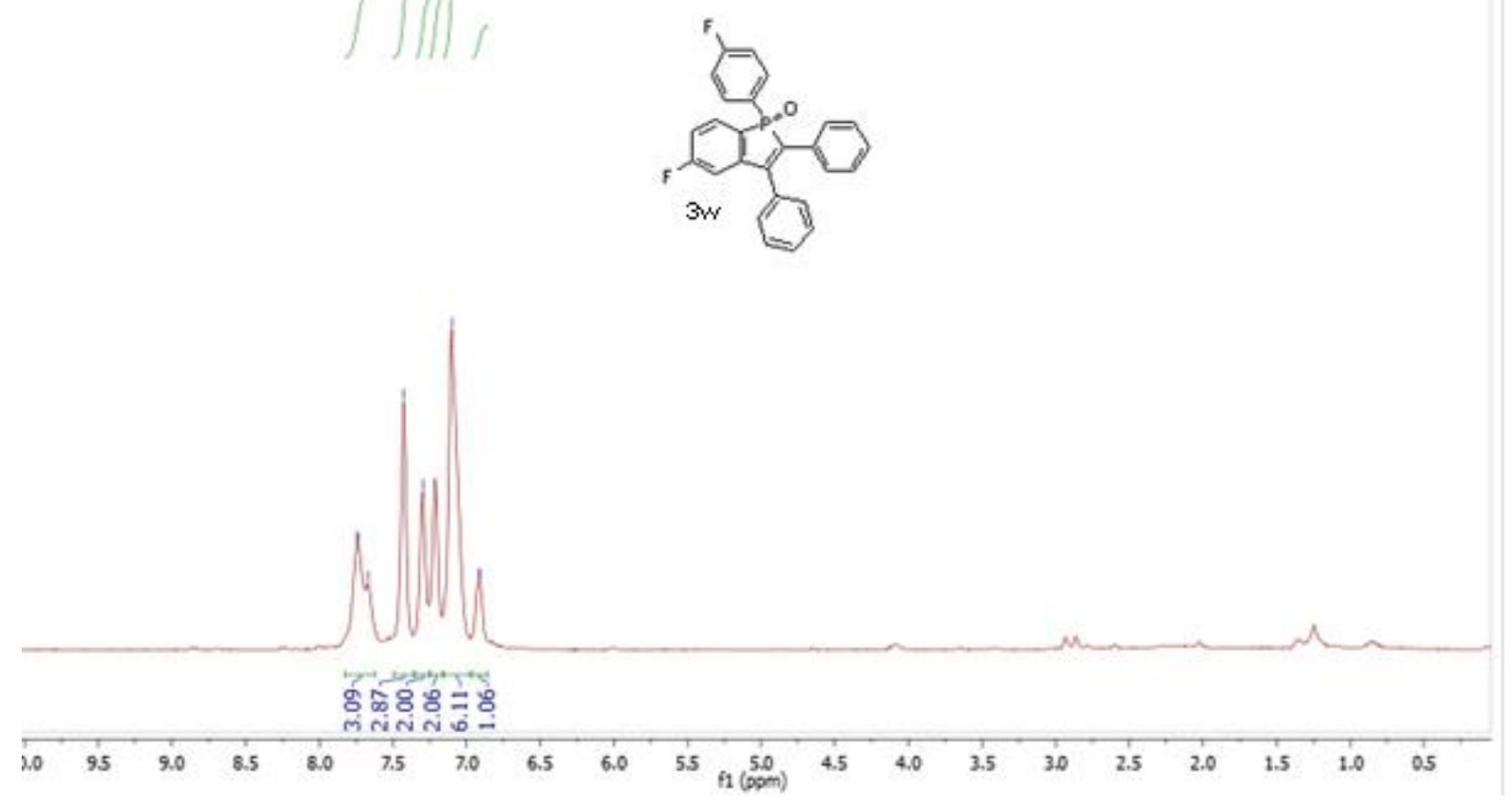

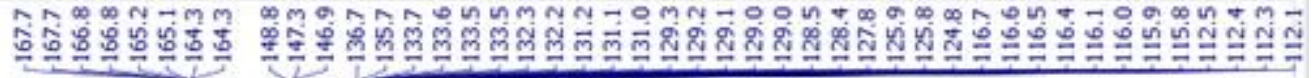

$\left({ }^{13} \mathrm{C}, 100 \mathrm{MHz}, \mathrm{CDCl}_{3}\right)$

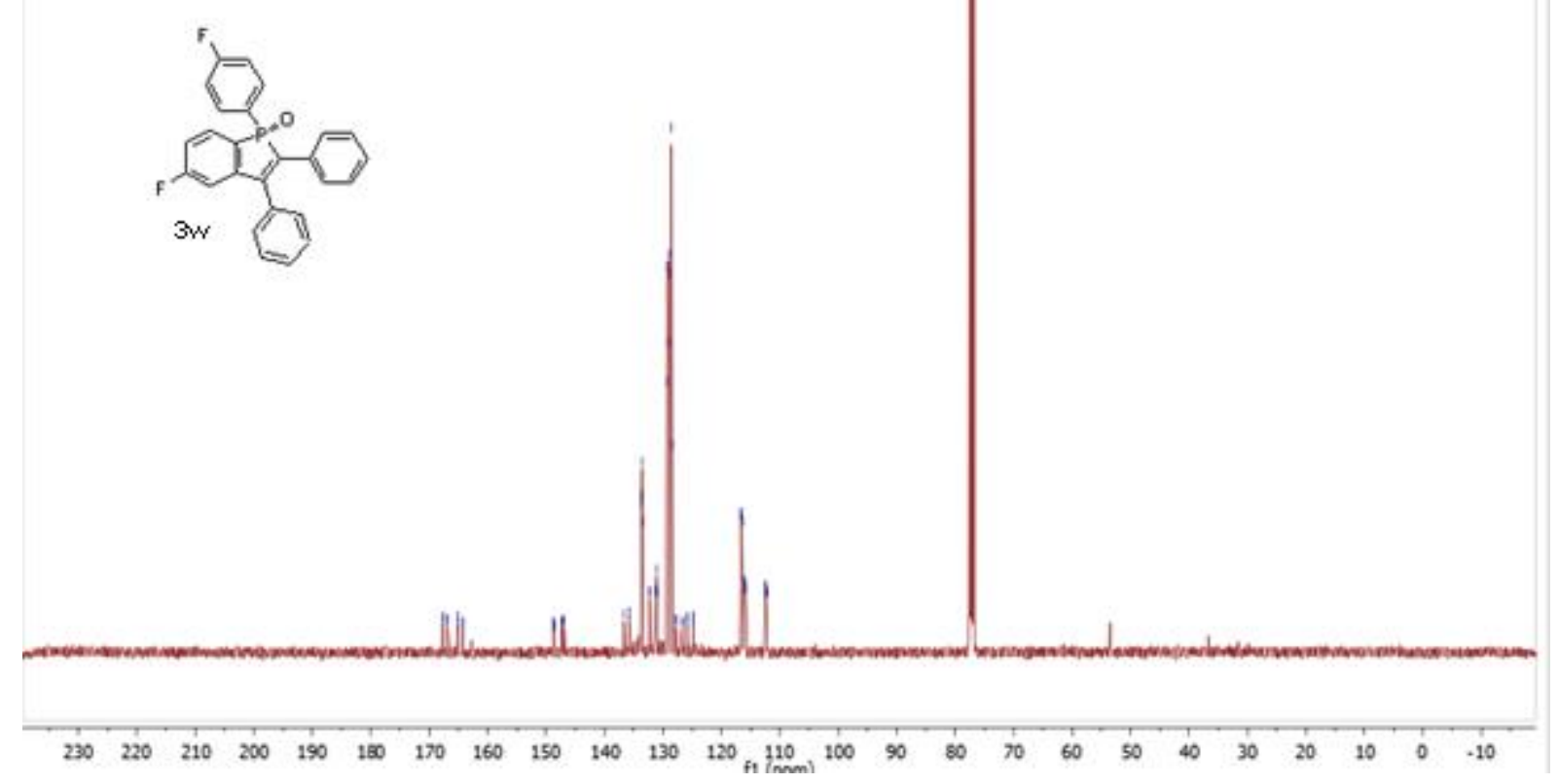


$\hat{\phi}$

$\left({ }^{31} \mathrm{P}, 162 \mathrm{MHz}, \mathrm{CDCl}_{3}\right)$

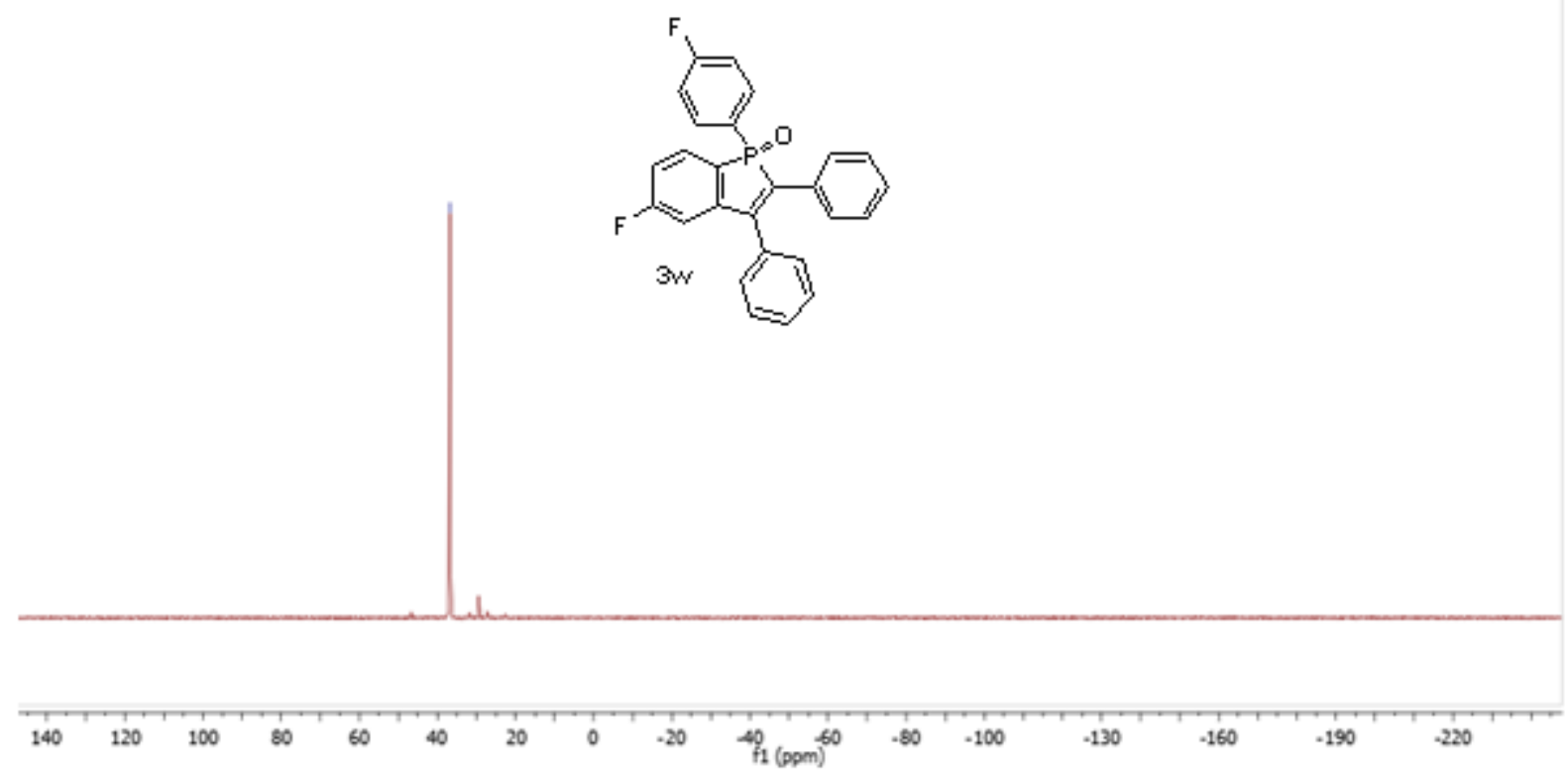

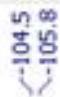

$\left({ }^{19} \mathrm{~F}, 362 \mathrm{MHz}, \mathrm{CDCl}_{3}\right)$

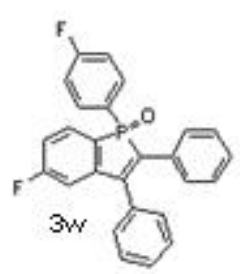

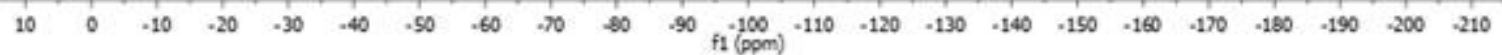




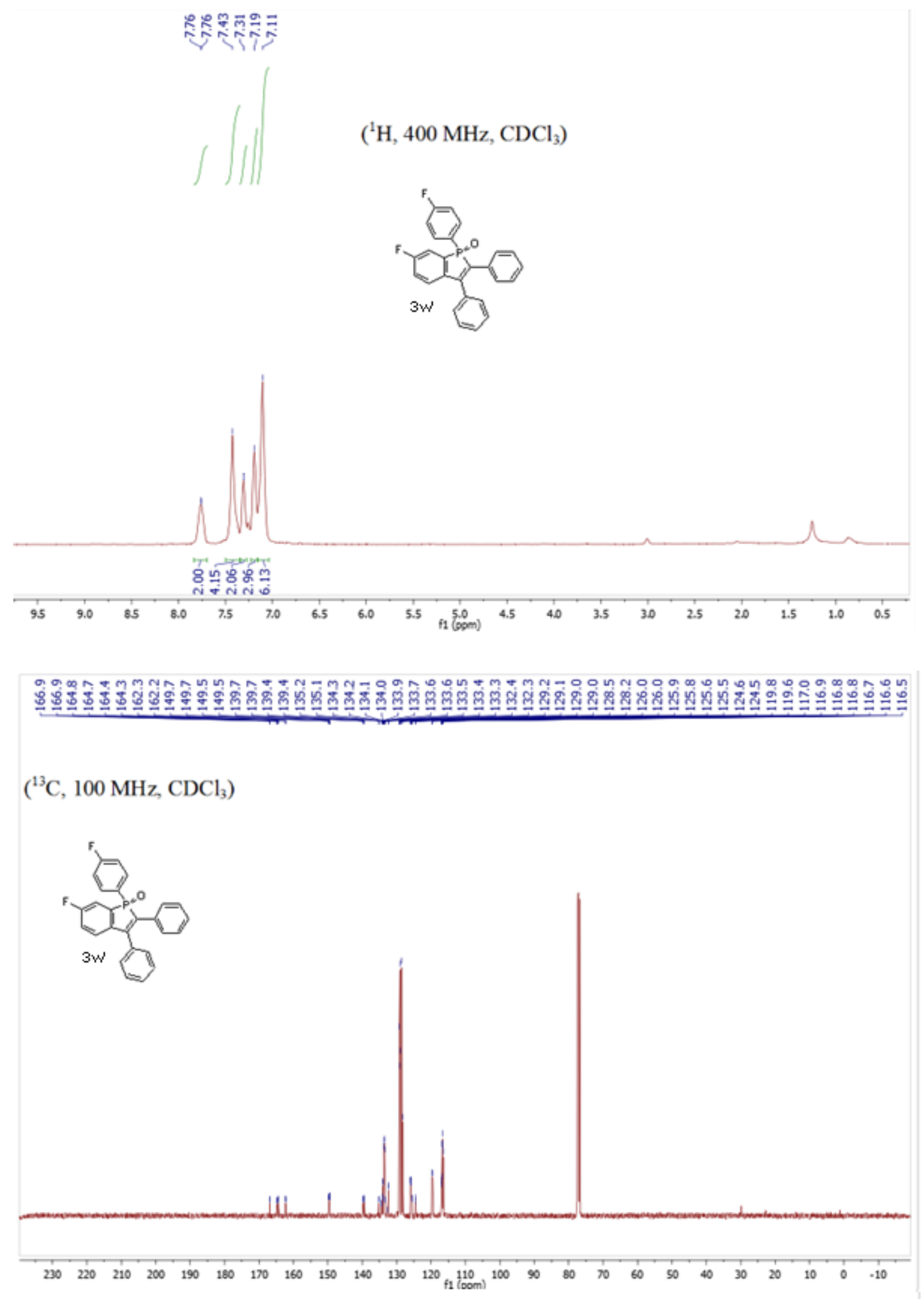



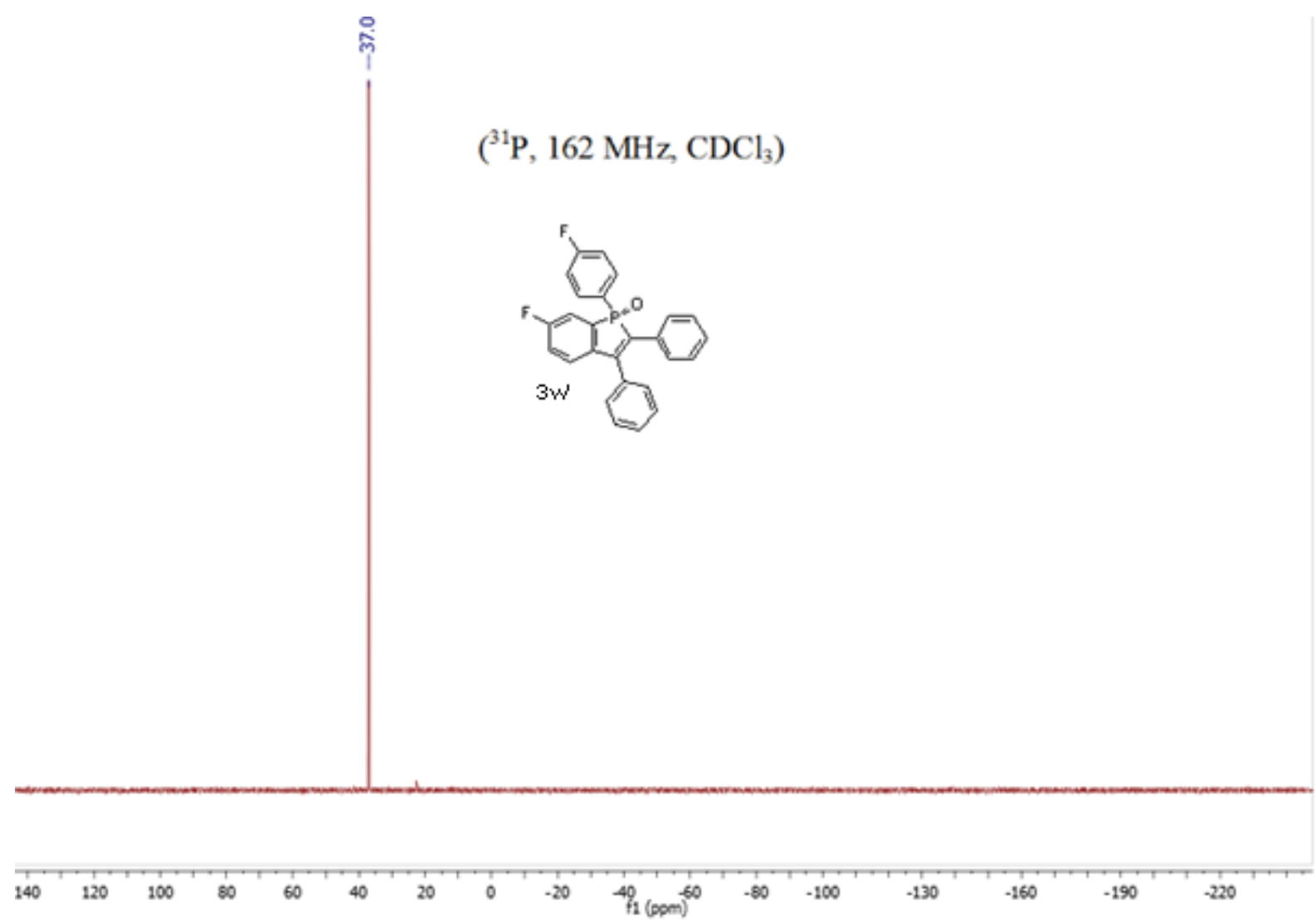

ํㅜㅇ음

$\left({ }^{19} \mathrm{~F}, 362 \mathrm{MHz}, \mathrm{CDCl}_{3}\right)$

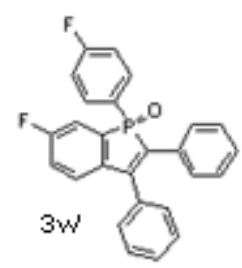

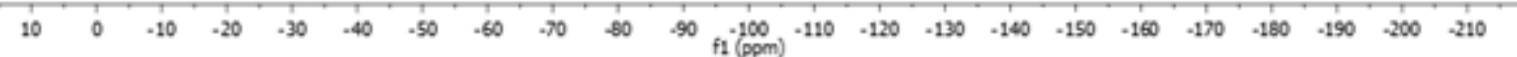




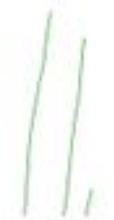

$\left({ }^{1} \mathrm{H}, 400 \mathrm{MHz}, \mathrm{CDCl}_{3}\right)$

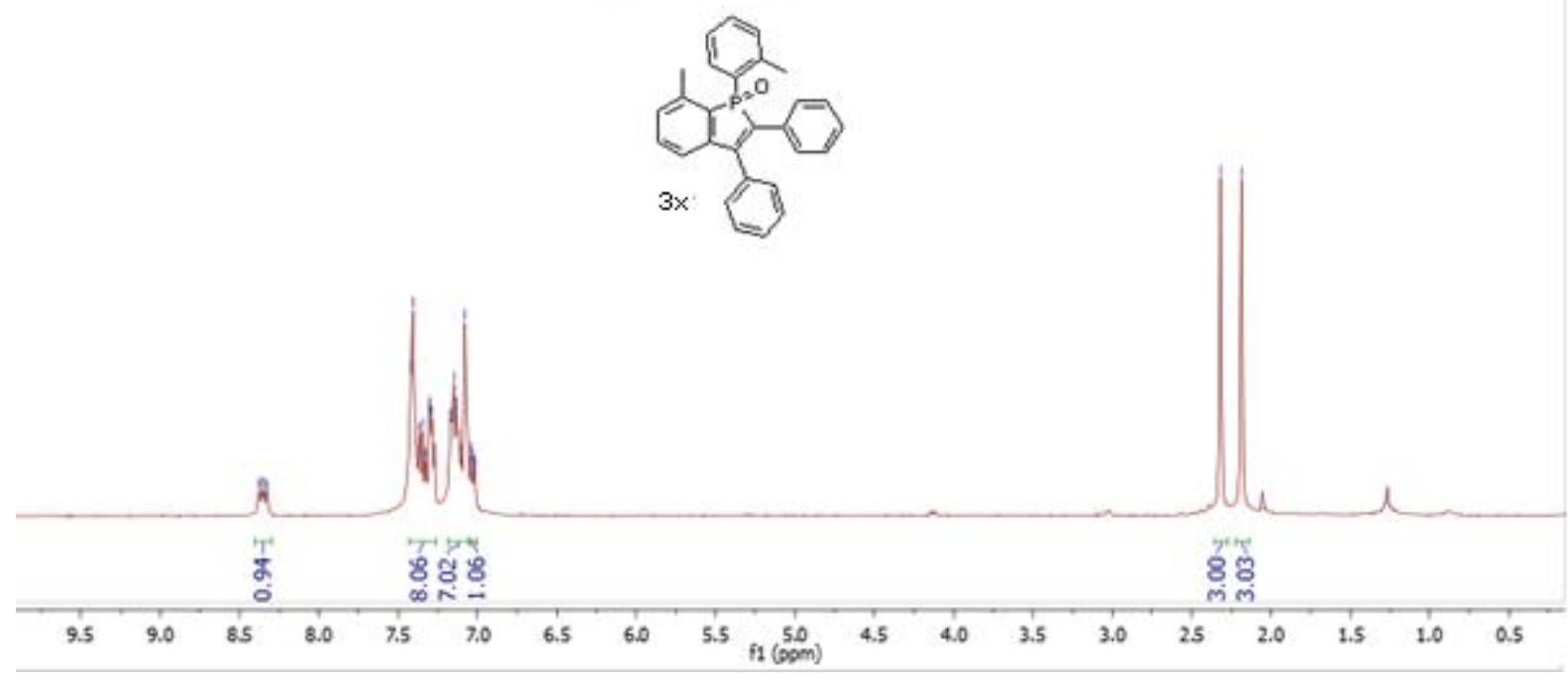

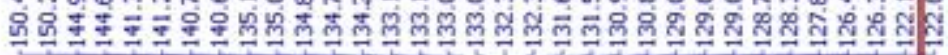

$\left({ }^{13} \mathrm{C}, 100 \mathrm{MHz}, \mathrm{CDCl}_{3}\right)$
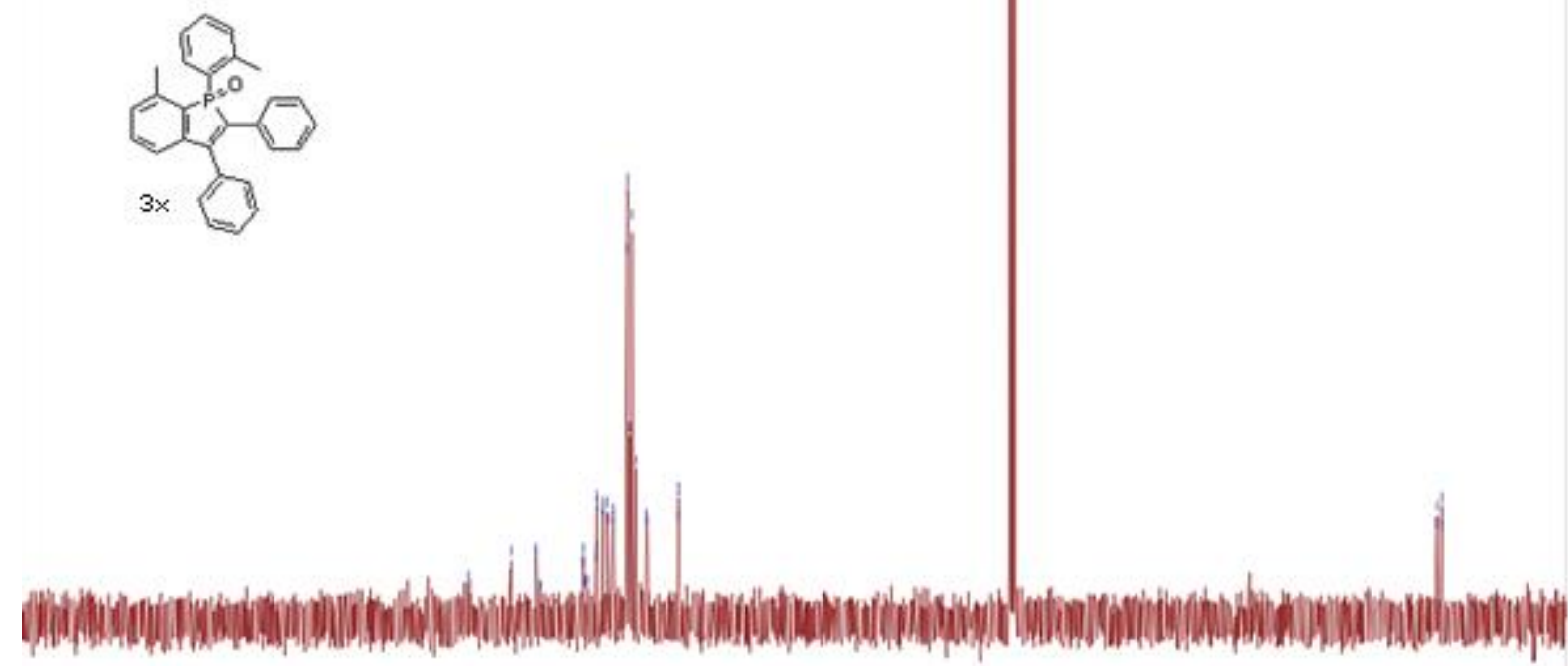

$\begin{array}{lllllllllllllllllllllllll}10 & 200 & 190 & 180 & 170 & 160 & 150 & 140 & 130 & 120 & 110 & 100 & 90 & 80 & 70 & 60 & 50 & 40 & 30 & 20 & 10 & 10\end{array}$ 

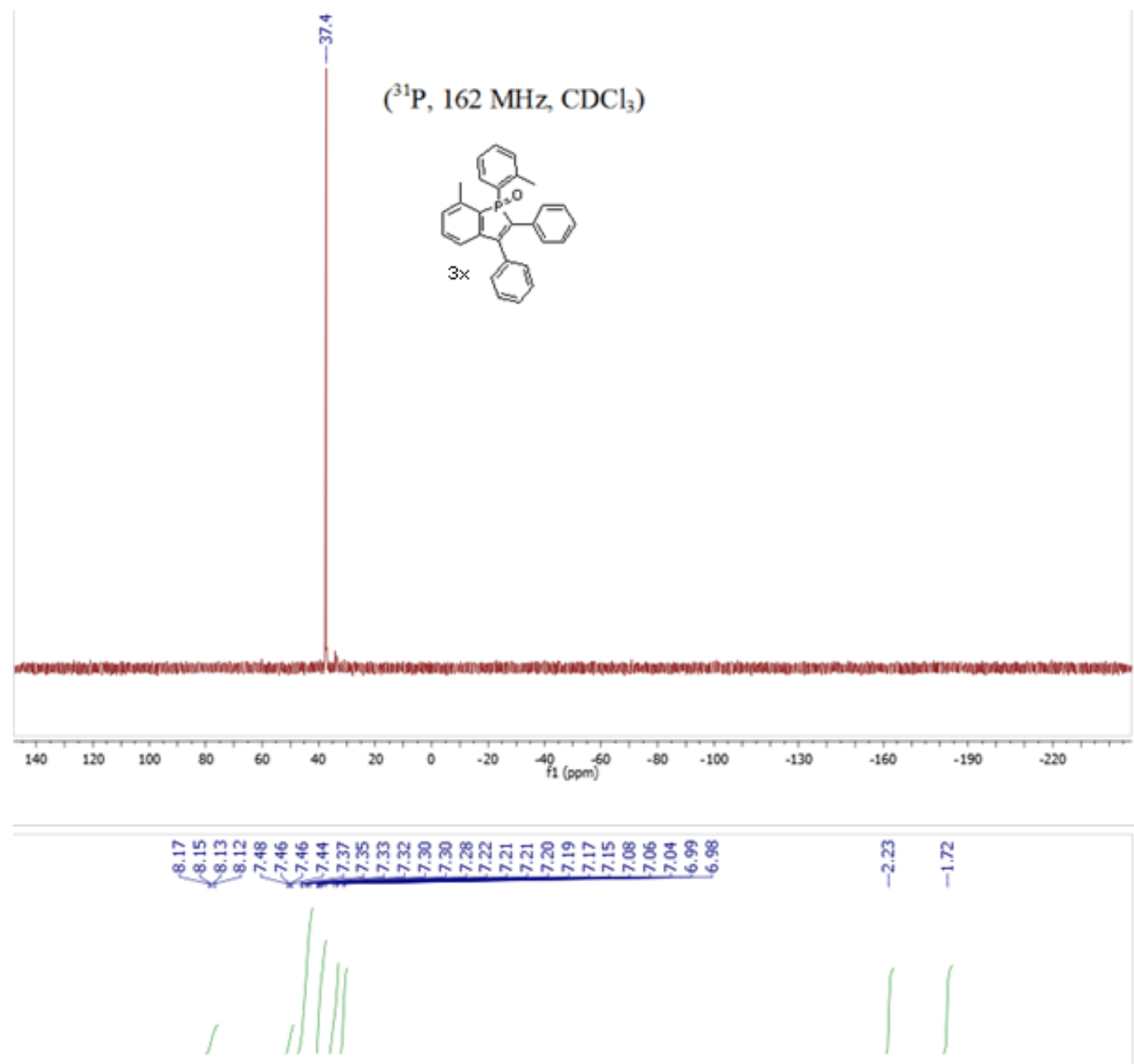

$\left({ }^{1} \mathrm{H}, 400 \mathrm{MHz}, \mathrm{CDCl}_{3}\right)$

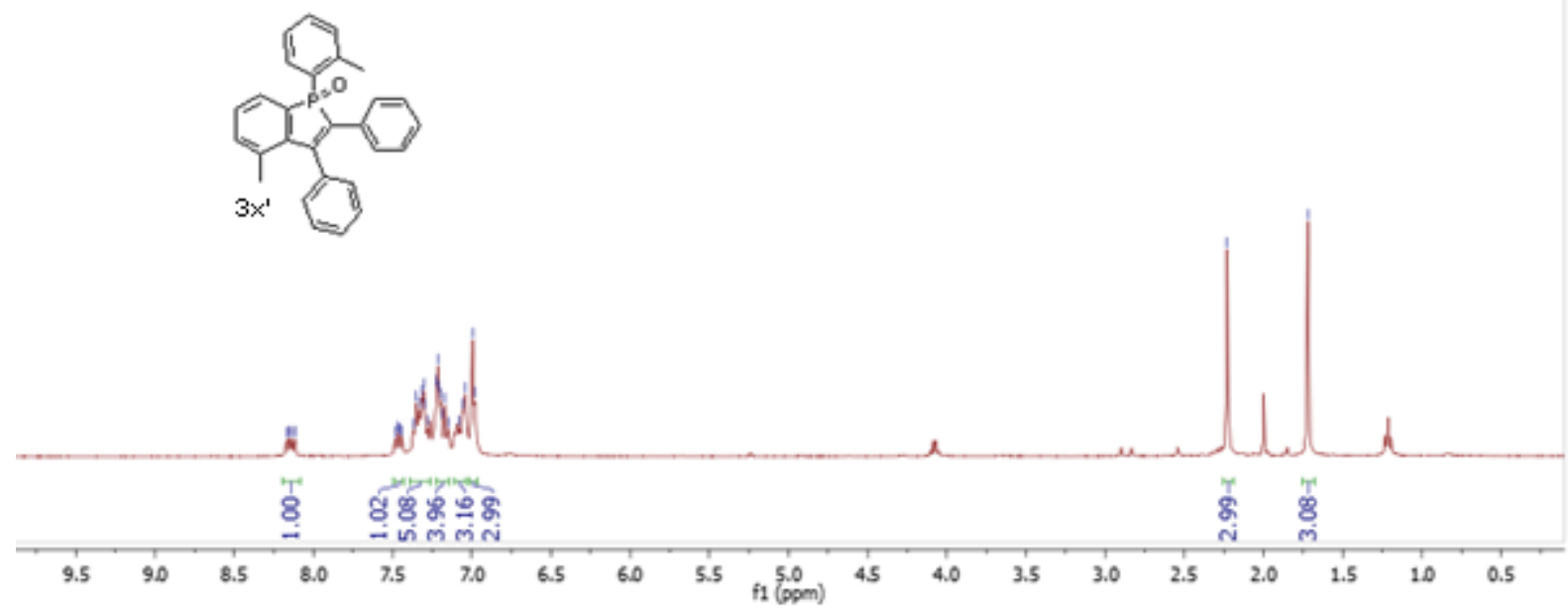




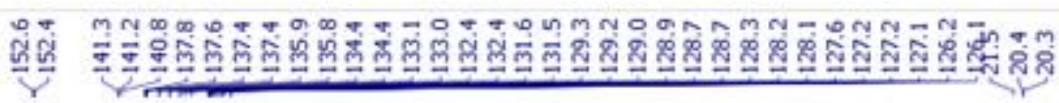

$\left({ }^{13} \mathrm{C}, 100 \mathrm{MHz}, \mathrm{CDCl}_{3}\right)$

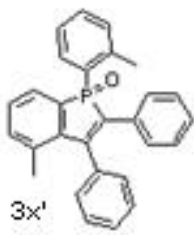
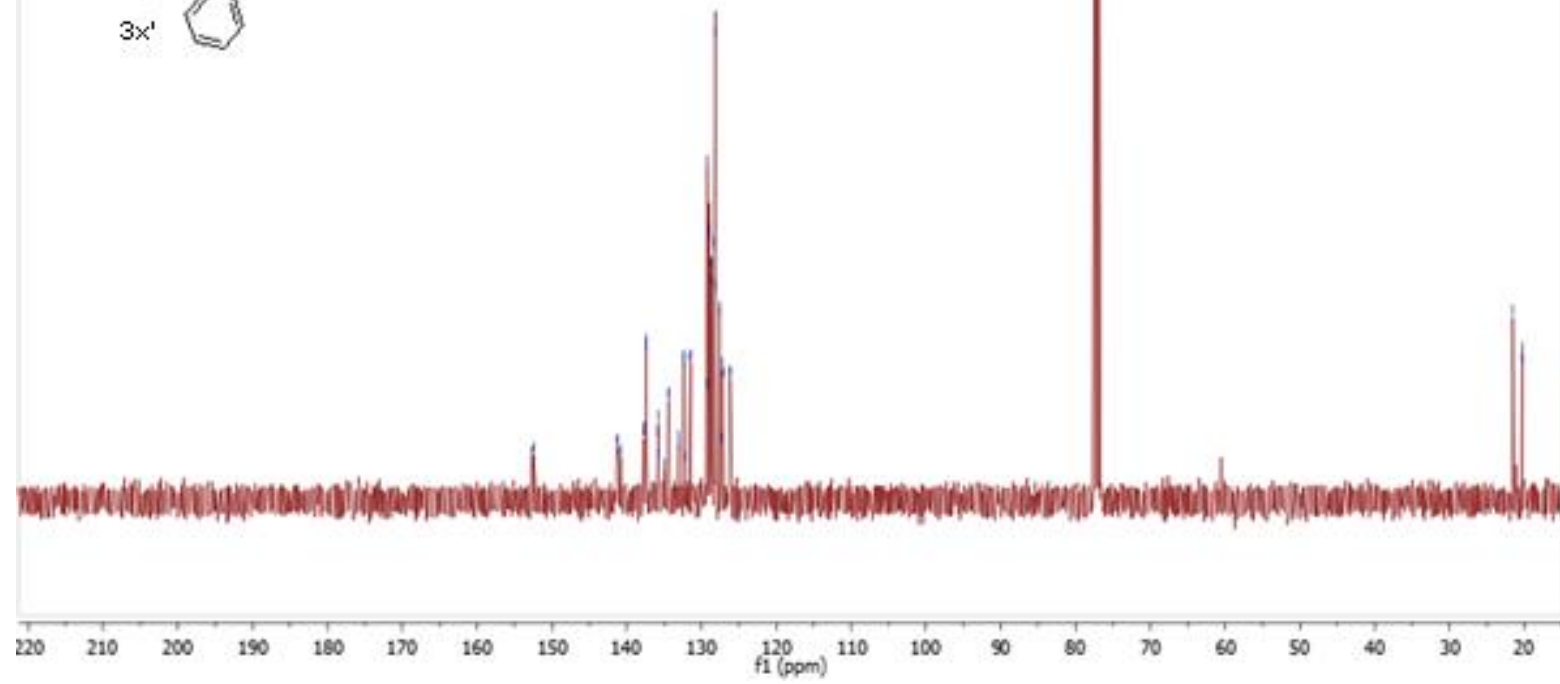

ॠ

$\left({ }^{31} \mathrm{P}, 162 \mathrm{MHz}, \mathrm{CDCl}_{3}\right)$

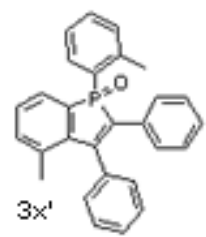

$\begin{array}{llllllllllll}140 & 120 & 100 & 80 & 60 & 40 & 20 & 0 & -20 & 40 & 60\end{array}$ 